Additional services for Journal of Fluid Mechanics:

Email alerts: $\underline{\text { Click here }}$

Subscriptions: $\underline{\text { Click here }}$

Commercial reprints: $\underline{\text { Click here }}$

Terms of use : $\underline{\text { Click here }}$

Short-scale break-up in unsteady interactive layers: local development of normal pressure gradients and vortex wind-up

L. LI, J. D. A. WALKER, R. I. BOWLES and F. T. SMITH

Journal of Fluid Mechanics / Volume 374 / November 1998, pp 335 - 378

DOI: 10.1017/S0022112098002626, Published online: 08 September 2000

Link to this article: http://journals.cambridge.org/abstract_S0022112098002626

How to cite this article:

L. LI, J. D. A. WALKER, R. I. BOWLES and F. T. SMITH (1998). Short-scale break-up in unsteady interactive layers: local development of normal pressure gradients and vortex wind-up. Journal of Fluid Mechanics, 374, pp 335-378 doi:10.1017/ S0022112098002626

Request Permissions : $\underline{\text { Click here }}$ 
(C) 1998 Cambridge University Press

\title{
Short-scale break-up in unsteady interactive layers: local development of normal pressure gradients and vortex wind-up
}

\author{
By L. LI ${ }^{1}$ ，J. D. A. W A L KER ${ }^{2}$, R. I. BOWLES $\mathbf{S}^{1}$ \\ AND F. T. S MIT H $\mathbf{H}^{1}$ \\ ${ }^{1}$ Department of Mathematics, University College London, Gower Street, London WC1E 6BT, UK \\ ${ }^{2}$ Department of Mechanical Engineering, Packard Laboratory No. 19, Lehigh University, \\ Bethlehem, PA 18015, USA
}

(Received 21 January 1997 and in revised form 2 March 1998)

Following the finite-time collapse of an unsteady interacting boundary layer (step 1), shortened length and time scales are examined here in the near-wall dynamics of transitional-turbulent boundary layers or during dynamic stall. The next two steps are described, in which (step 2) normal pressure gradients come into operation along with a continuing nonlinear critical-layer jump and then (step 3) vortex formation is induced typically. Normal pressure gradients enter in at least two ways, depending on the internal or external flow configuration. This yields for certain internal flows an extended $\mathrm{KdV}$ equation with an extra nonlinear integral contribution multiplied by a coefficient which is proportional to the normal rate of change of curvature of the velocity profile locally and whose sign turns out to be crucial. Positive values of the coefficient lead to a further finite-time singularity, while negative values produce a rapid secondary instability phenomenon. Zero values in contrast allow an interplay between solitary waves and wave packets to emerge at large scaled times, this interplay eventually returning the flow to its original, longer, interactive, boundary-layer scales but now coupled with multiple shorter-scale Euler regions. In external or quasiexternal flows more generally an extended Benjamin-Ono equation holds instead, leading to a reversal in the roles of positive and negative values of the coefficient. The next step, 3 , typically involves the strong wind-up of a local vortex, leading on to explosion or implosion of the vortex. Further discussion is also presented, including the three-dimensional setting, the computational implications, and experimental links.

\section{Introduction}

There are two major areas of practical interest that motivate the current work, mainly concerning internal or external aerodynamics but with application also to atmospheric, physiological and machinery dynamics among others. One is dynamic stall, for example on pitching airfoils, turbine blades, or in unsteady internal flows, and the other is transition from laminar to turbulent flow, on airfoils, flat surfaces or in channels. Both have been subject to much experimental and computational study over the years, e.g. see reviews by C. R. Smith et al. (1991), Doligalski, Smith \& Walker (1994), Smith (1993, 1995), references therein, and Lorber \& Carta (1988). Both areas involve very complex fluid dynamics, at large Reynolds numbers, with various disparate length and time scales present, as typically for external flows a 
boundary layer is lifted away from the airfoil surface, accompanied by vortex roll-up and ejection into the free stream outside. In consequence it is extremely difficult, if not impossible, with present-day methods, to compute solutions for the flow fields accurately at the large Reynolds numbers of real practical interest. It seems fairly clear that theoretical backing which takes proper account of the largeness of the Reynolds number is extremely desirable in both of these areas.

The present study is aimed at the large gap that exists, in these areas, between the capabilities of accurate direct numerical simulations and the practical needs which tend to be at much larger Reynolds number. There is a similar gap to be bridged between studies of small-scale unsteady separation and the modelling of larger-scale unsteady separation with vortex shedding, a gap that is addressed here based initially on nonlinear singularities or break-ups in unsteady classical and/or interactive boundary layers and internal flows. In the transition setting we address break-up and the development of short-scale processes in the near-wall dynamics of internal or external transitional boundary layers. The near-wall dynamics also appears to be a key element in the make-up of turbulent boundary layers: see for example Doligalski et al. (1994), Walker et al. (1989), Walker $(1990 a, b)$. This and other theoretical aspects of turbulent boundary layers based on rational arguments are examined by Smith, Doorly \& Rothmayer (1990), Smith, Dodia \& Bowles (1994), Walker (1990a), C. R. Smith et al. (1991) (and references therein), including renormalization properties, sub-scales, spots and displacement-thickness enlargement. The trend of the current theoretical and numerical work is presented below in terms of consecutive steps, $0,1,2,3, \ldots$. There are many possible scales and particular contexts but all have a common theme.

In the context of dynamic stall, there are again several possible configurations and scales involved, but many local flow features are virtually the same as for transition. In motion due to an impulsively started thick airfoil or a pitching airfoil, the attached classical unsteady boundary layer approaches the Van Dommelen (1981) singularity, first, near which interaction between the pressure and the displacement takes place, second: Elliott, Cowley \& Smith (1983), Peridier, Smith \& Walker $(1991 a, b)$, Adams, Conlisk \& Smith (1995), Cassel, Smith \& Walker (1996). The action of the classical unsteady boundary layer is regarded here as a 'step 0 ', prior to the pressuredisplacement-interactive stage which is 'step 1'. Cassel et al. point out a delicate dependence in the onset of interaction on the initial conditions. An alternative step 0 is associated with unsteady marginal separation (Smith 1982; Elliott \& Smith 1985, 1987; Timoshin 1988), bringing in full pressure-displacement interaction next as the step 1. Similar situations occur in transition, as we see below. Thus the unsteady interactive step 1 represents a common thread throughout.

So $\$ 2$ below starts with step 1, the unsteady interacting-boundary-layer system, the two-dimensional version forming a central starting point (cf. the three-dimensional version, which is considered by Hoyle \& Smith 1994). This nonlinear stage often corresponds to nonlinear Tollmien-Schlichting (TS) wave development, in the context of transition, given that the linearized system captures linear TS waves (Smith 1979 and related references below). In the dynamic-stall context, the interacting stage is encountered due to or even prior to (Cassel et al.) the local eruption in the classical unsteady boundary-layer stage. Likewise, during transition, there can again be a prior step 0, as above (Smith \& Burggraf 1985; Smith et al. 1994, see also Zhuk \& Ryzhov 1982) or as a vortex-wave interaction (Smith \& Walton 1989; Hall \& Smith 1991; Smith \& Bowles 1992; Walton \& Smith 1992; Smith, Brown \& Brown 1993). The finite-time break-up of the interactive step 1 is summarized in $\$ 2$ below, based 
on the theory in Smith (1988a), with Appendix A verifying its generality. (See also computational and other aspects in Peridier et al. 1991a,b; Hoyle, Smith \& Walker 1991; Adams et al. 1995). That leads on to the next stage, step 2, which is the basis of this study and whose analysis starts in $\$ 3$. There normal pressure gradients and a developed nonlinear critical-layer jump (cf. Dickinson 1970; Stewartson 1978; Gajjar \& Smith 1985; Haynes \& Cowley 1986; Smith \& Bodonyi 1987) come into play locally. Normal pressure gradients can enter in at least two ways: via a displacement feedback in the outer uniform-shear motion for the external (or quasi-external) context or directly from the normal momentum balance for certain internal-flow contexts. These latter contexts yield an extended Korteweg-de Vries (KdV) equation for the main pressure response. The extension from the $\mathrm{KdV}$ equation involves an extra integral term which is due to a critical-layer jump and has a constant coefficient $\mu$. The key parameter $\mu$ itself is proportional to the normal rate of change of the velocity-profile curvature in the neighbourhood of the critical layer.

The cases of positive $\mu$, negative $\mu$ and zero $\mu$ are examined separately in $\S \S 4-6$, respectively, as they possess distinct characteristics. The first leads in to a further finite-time irregularity and the second to a strong secondary instability. Thus before the traditional soliton-containing stage normally associated with a KdV equation can be reached more new physics comes into play in general. The special case of zero $\mu$ however continues to large scaled times, acquiring then an intriguing structure with solitary and travelling waves ( $\S 6$ ). In the case of positive $\mu$ by contrast ( $\$ 4)$ the subsequent stage or step 3, which is considered in $\$ 7$, is due essentially to the formation of an increasingly strong, spanwise, vortex winding up, within the nonlinear critical layer, together with faster time scales and still shorter length scales. The further increase or decrease of the vortex strength is studied in $\S 7$. It is interesting to observe that the criterion for the occurrence of the winding-up vortex here concerns the onset of a maximum/minimum in the scaled local pressure variation $\tilde{p}$ (see also Hoyle et al. 1991),

$$
\tilde{p}_{X} \rightarrow 0,
$$

along with an integral condition on the local velocity profile $u_{0}(y)$, namely

$$
f_{0}^{\infty}\left[u_{0}(y)-c\right]^{-2} \mathrm{~d} y=0
$$

(Smith 1988a) for the effective local phase speed $c$, where the coordinate $y$ is in the normal direction. (In (1.2) the upper limit of integration should be taken at an upper boundary or symmetry line where appropriate, for instance in certain internal motions.) The theoretical criterion (1.2) is met fairly closely in the channel-flow transition experiments of Nishioka, Asai \& Iida (1979) as quantitative comparisons in Smith \& Bowles (1992) show (see also Savin 1996; Smith 1996; Savin, Smith \& Allen 1998), while both (1.1), (1.2) are found to be met approximately in the computations of Sandham \& Kleiser (1992) (private communications 1991-94 with Dr N. Sandham are gratefully acknowledged) and Jones \& Fiddes (1994, private communication) and Jones (1994). Again, (1.1) is used by Wintergerste \& Kleiser (1995) as a measure of the location of spikes or vortices near the end of transition in their direct simulations. The criteria, then, according to the above evidence, correspond to the formation of the so-called 'first spike' (at least) during certain transition paths and possibly to Klebanoff-type breakdown (Schubauer \& Klebanoff 1956; Klebanoff \& Tidstrom 1959; Klebanoff Tidstrom \& Sargent 1962).

Further discussion is presented in $\S 8$. This includes the alternative external or 
quasi-external case of the extended Benjamin-Ono equation: for, although $\S \S 4-7$ concentrate on the simpler, extended KdV, balance for special internal flows, the extended Benjamin-Ono balance in fact has more general application, and so its analogous properties are summarized in $\S 8$, these indicating a role reversal for positive and negative values of $\mu$. Also considered in $\S 8$ are the extension to the three-dimensional version; connections with experiments and simulations; and the implications for computational studies of internal or external dynamic stall and transition.

Non-dimensional variables are adopted, with the velocity $(\bar{u}, \bar{v})$ in $(\bar{x}, \bar{y})$ Cartesian coordinates (streamwise, normal), the convective time $\bar{t}$ and the pressure $\bar{p}$ being such that the non-dimensional airfoil chord, say, is unity, as is the characteristic free-stream speed for external motions. The corresponding global Reynolds number Re is large, and the typical undisturbed boundary-layer thickness is then of order $R e^{-1 / 2}$. For the case of internal motions the typical channel width, say, and the undisturbed flow speed are both unity. Some of the scales that appear subsequently, as we go beyond step 1 into steps 2, 3, may seem rather bizarre at first sight but the underlying flow characteristics as indicated earlier are believed to be quite clear physically. We address the incompressible regime, for definiteness, although the local dynamics is virtually independent of the pressure-displacement law; it is also predominantly inviscid, granted that by then viscosity has already had a profound effect during step 0 and/or step 1 in producing a critical profile $u_{0}(y)$ satisfying (1.2).

\section{Finite-time break-up in unsteady interacting boundary layers (or the nonlinear TS stage): step 1}

Much work has been done theoretically and computationally on unsteady external and internal boundary layers, in both the interactive ( $\bar{p}$ unknown) and the noninteractive ( $\bar{p}$ prescribed) regimes. The present research starts with the interactive case, regarded as step 1, a case which is both interesting and very generally useful, with its capturing of linear TS waves in the linear regime for triple-deck scales and its applications to transition and possible intermittency (see later), among other things, in the nonlinear regime. Here we work mostly in terms of the incompressible tripledeck case as our beginning, corresponding directly to the TS scales (see references in §1), and extend to internal flow subsequently. Thus in the lower deck or viscous sublayer, which is the main concern in the nonlinear TS stage, the flow solution has the expansion

$$
[\bar{u}, \bar{v}, \bar{p}]=\left[\epsilon u, \epsilon^{3} v, \epsilon^{2} p(x, t)\right]+\ldots, \quad[\bar{x}, \bar{y}, \bar{t}]=\left[\bar{x}_{0}+\epsilon^{3} x, \epsilon^{5} y, \epsilon^{2} t\right]
$$

near a typical station $\bar{x}=\bar{x}_{0}$, with the traditional factor $\epsilon \equiv R e^{-1 / 8}$ being small. An extension can be made to more general local length scales $\ell$ between $O\left(\epsilon^{3}\right)$ and $O\left(\epsilon^{6}\right)$, such that the successive factors in $(2.1 a, b)$ become $\delta, \delta^{2} R e^{-1 / 2} \ell^{-1}, \delta^{2}, \ell, \delta R e^{-1 / 2}, \ell \delta^{-1}$ in turn; here a viscous-inviscid balance requires $\delta=\ell^{1 / 3}$ and the range covered below is then $\epsilon^{2} \ll \delta \leqslant O(\epsilon)$, although that can be extended further if viscous effects are entirely absent. Given $(2.1 a, b)$ the governing equations here therefore are the scaled planar unsteady boundary-layer equations, in a standard notation,

$$
\begin{gathered}
u=\psi_{y}, \quad v=-\psi_{x}, \\
u_{t}+u u_{x}+v u_{y}=-p_{x}(x, t)+u_{y y},
\end{gathered}
$$

subject to the boundary conditions

$$
u=\psi=0 \text { at } y=0,
$$




$$
u \sim y+A(x, t) \text { as } y \rightarrow \infty .
$$

The $\bar{y}$-momentum balance confirms that the scaled pressure $p$ is independent of $y$, and both $p$ and the negative displacement $A$ are unknown function of $x, t$. Among the boundary conditions required to complete the system, the pressure-displacement law depends on the particular context of interest, examples being the Cauchy-Hilbert relation for subsonic external flows and Ackeret's law in supersonic external flows. The former applies in the present setting $(2.1 a, b)$ of an incompressible boundary layer, in the form

$$
p(x, t)=\frac{1}{\pi} f_{-\infty}^{\infty} \frac{A_{\chi}(\chi, t) \mathrm{d} \chi}{(x-\chi)}
$$

from the potential-flow properties holding in the upper deck, of thickness $O\left(\epsilon^{3}\right)$ in $\bar{y}$, outside the $O\left(\epsilon^{4}\right)$ thick boundary layer. There are many other contexts of interest yielding other interaction laws, as noted by Smith (1988b), with the right-hand side of (2.3c) replaced by $\pm A,-A_{x},-A_{x x}$ for hypersonic-limit or liquid-layer flows, supersonic boundary layers and wall jets, in turn, while short-scale interactions simply have $A \equiv 0$, e.g. for $\delta$ less than $O(\epsilon)$. Various combinations are also encountered in some flow configurations; see e.g. Brotherton-Ratcliffe (1986), Bowles \& Smith (1992), Brown, Cheng \& Lee (1990) and below. Likewise, there are several useful interpretations at finite $R e$. Finally here, an internal-flow context of particular concern is for symmetric channel motion on an $O(R e)$ length scale, such that

$$
[\bar{u}, \bar{v}, \bar{p}, \bar{x}, \bar{y}, \bar{t}] \sim\left[u, \operatorname{Re}^{-1} v, p, x \operatorname{Re}, y, t R e\right] .
$$

There $(2.2 a, b),(2.3 c)$ hold again but subject to a mass-flow constraint

$$
\psi=\text { constant, } u_{y}=0 \text { at } y=a,
$$

where $a$ is the channel half-width.

In all contexts, however, a finite-time break-up is possible according to the theory in Smith (1988a), for the initial-value problem. The type of break-up singularity that is probably the most common is the so-called moderate type, in which the length scale

$$
x-x_{0}=c\left(t-t_{0}\right)+\left(t_{0}-t\right)^{3 / 2} \xi(\text { with } \xi \text { of } O(1))
$$

contracts near the break-up position $x=x_{0}$, at the break-up time $t=t_{0}^{-}$, and the scaled solution takes the form

$$
[u, \psi, p]=\left[u_{0}(y), \psi_{0}(y), p_{0}\right]+\left(t_{0}-t\right)^{1 / 2}\left[u_{1}(\xi, y), \psi_{1}(\xi, y), p_{1}(\xi)\right]+0\left(t_{0}-t\right)^{3 / 4},(2.4 b-d)
$$

where $p_{0}$ is constant and the characteristic $y$ value remains $O(1)$. Thinner sublayers are brought into action near the critical layer, where $u_{0}$ is equal to the phase speed $c$ and a nonlinear critical layer is provoked, and near the wall where a viscous wall layer is required, but these need not concern us in detail yet; see Smith (1988a), and compare with the subsequent sections. Analysis of (2.2) with the local expansions (2.4) then leads (see last reference) to a nonlinear equation, $p_{1} p_{1}^{\prime}=\hat{b}\left(p_{1}-3 \xi p_{1}^{\prime}\right)$, for $p_{1}(\xi)$ with $\hat{b}$ being a constant which, like the phase speed $c$, depends on integral properties of the local velocity profile $u_{0}(y)$ at $x=x_{0}$, cf. (3.6), (3.7) below. An assumed property concerning the coefficient $\hat{b}$ implicit in the last paper is verified in Appendix A to be generally true, and we should also observe that the critical-layer jump here works out to be zero, cf. the next section, while the higher-order terms in $(2.4 b-d)$ are dependent on the particular context, as a comparison with Brotherton-Ratcliffe \& Smith (1987) shows. The $p_{1}$ distribution above can be obtained explicitly (see also 
$\S \S 3,4$ and in particular (4.4)). Hence a singularity in the pressure gradient is predicted, with $p-p_{0} \propto\left|x-x_{0}\right|^{1 / 3}$ locally at the time $t=t_{0}$.

Computations of the unsteady interactive-boundary-layer development due to a vortex above a plane wall (Peridier et al. 1991b), using a Lagrangian scheme, up to the evolution of a singularity, provide strong support for the theoretical account summarized in (2.4) and figure $1(a, b)$. We add in passing that there is a whole range of possible singular forms (see Smith 1988a), all predominantly inviscid, stretching from the moderate one above to a severe one in which $p$ itself is discontinuous, analogous to the singularities of the inviscid Burgers equation. For now we concentrate below on the repercussions of the main break-up type (2.4) and the new physical properties necessarily entering locally, as time advances.

\section{The intrusion of normal pressure gradients: step 2}

An order-of-magnitude argument indicates that, closer to the above singular position and time $\left(x_{0}, t_{0}\right)$, new physical influences come into play next which are associated predominantly with normal-pressure-gradient effects as well as a development of a new nonlinear critical-layer jump contribution. Normal pressure gradients can enter in at least two ways, and here we address mainly the two representative configurations $(2.3 c)$ for triple-deck external flow and $(2.3 e)$ for internal flow. The length and time scales turn out to be reduced by factors $\epsilon^{3 / 2}, \epsilon$ for the triple-deck context and $R e^{-6 / 7}, R e^{-4 / 7}$ for the internal-flow context, in the relevant moving frame, so that

$$
\bar{x}=\text { constant }+\delta_{1} \delta_{3} c T+\delta_{5} X, \quad \bar{t}=\text { constant }+\delta_{3} T,
$$

where $\left(\delta_{1}, \delta_{3}, \delta_{5}\right)$ denotes $\left(\epsilon, \epsilon^{3}, \epsilon^{9 / 2}\right)$ for the external triple-deck case and $\left(1, R e^{3 / 7}\right.$, $R e^{1 / 7}$ ) for the internal case, other cases being similar. The scales here follow from an order-of-magnitude argument based on $\bar{p}_{\bar{y}}$ in step 1 being of order $R e^{-2}$ in the internal flow context (from $\bar{u} \bar{v}_{\bar{x}}$ evaluated using $(2.3 d)$ ) but then growing like $\left(t_{0}-t\right)^{-5 / 2}$ in view of $(2.4 a, d)$ as $t \rightarrow t_{0}-$. Thus the normal pressure variation then has typical size $R e^{-2}\left(t_{0}-t\right)^{-5 / 2}$. This becomes comparable with the solvability pressure level $O\left(t_{0}-t\right)$ in Smith (1988a) when $\left(t_{0}-t\right)^{7 / 2}$ decreases to $R e^{-2}$, which along with (2.3d) confirms (3.1b). The external scales follow similarly, the scales involved agreeing with those in Smith (1977, 1996), Savin (1996). For definiteness we will keep to these two internal and external cases below. There are two main tiers (i), (ii) now to investigate in the $\bar{y}$-direction, as indicated schematically in figure $1(b)$ and as implied by the break-up in $\S 2$, and an additional tier (iii) for the external case. There is also a viscous wall layer present but this in essence is passive (being controlled by a linear unsteady-viscous Stokes balance as in Smith 1988a), leaving the local dynamics predominantly inviscid.

The first tier (i) comprises the bulk of the local flow, in which the expansion holding becomes

$$
\begin{gathered}
\bar{u}=\delta_{1}\left[u_{0}(y)+\delta_{2} \tilde{u}(X, y, t)+\delta_{2}^{2} \tilde{u}_{2}(X, y, t)+\ldots\right], \\
\bar{v}=\delta_{1} \delta_{4} \delta_{5}^{-1}\left[\delta_{2} \tilde{v}(X, y, t)+\delta_{2}^{2} \tilde{v}_{2}(X, y, t)+\ldots\right], \\
\bar{p}=\delta_{1}^{2}\left[p_{0}+\delta_{2} \tilde{p}(X, t)+\delta_{2}^{2} \tilde{p}_{2}(X, y, t)+\ldots\right],
\end{gathered}
$$

for scaled $X, y, T$ of $O(1)$. Here $\bar{y}=\delta_{4} y$ with $\delta_{4}$ being $\epsilon^{5}$ in the external triple-deck context, but 1 in the internal-flow context, and $\delta_{2}$ denotes $\epsilon^{1 / 2}, R e^{-2 / 7}$ respectively. Analysis of the Navier-Stokes equations subject to the expansion (3.2) then leads to 
(a)

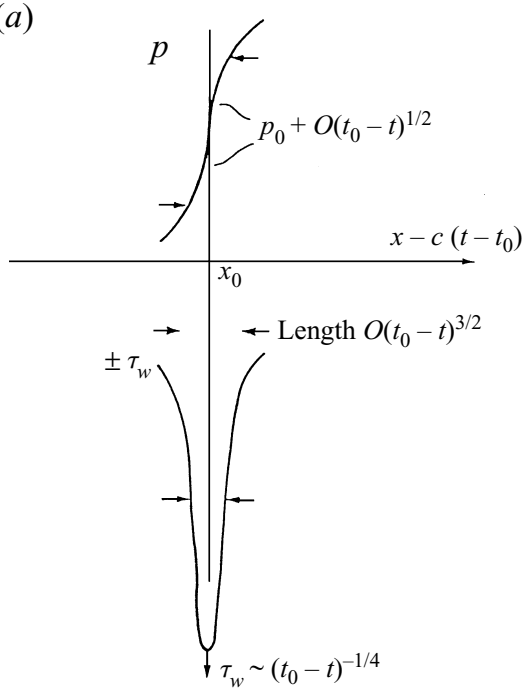

(b)

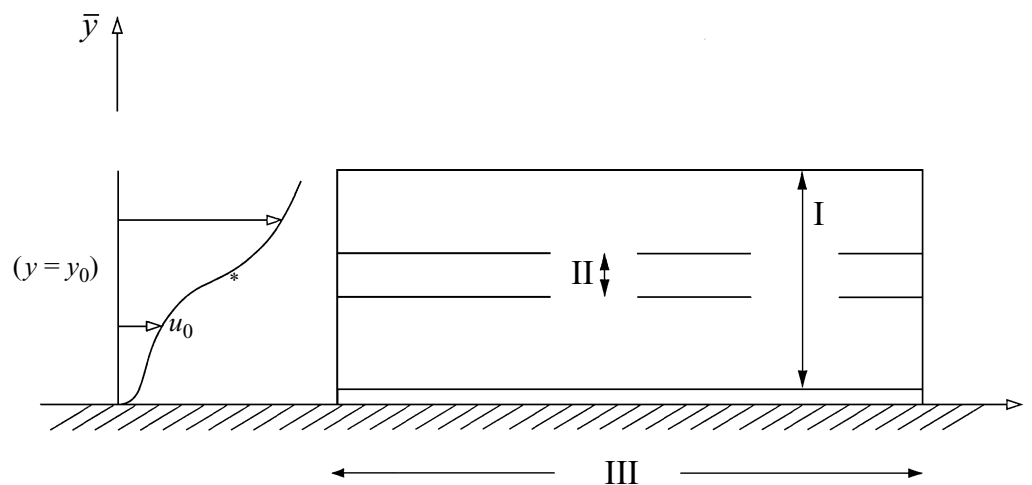

Figure 1. On steps 1, 2. (a) Schematic diagram of the local behaviour of the scaled pressure $p$ and wall shear $\tau_{w}$ near break-up (Smith 1988a) of step 1. (b) Flow structure, with inflection point $(*)$ developed in the profile $u_{0}$. Length scales I-III in the appropriate moving frame are as given in $\$ \S 2$, 3 . For the external-flow case the termination of step 1 has I-III equal to $\epsilon^{5}, \epsilon^{5}\left(t_{0}-t\right)^{1 / 4}, \epsilon^{3}\left(t_{0}-t\right)^{3 / 2}$ respectively, whereas in step 2 where normal pressure gradients become significant the scales I-III are $\epsilon^{5}(\operatorname{tier}(\mathrm{i})), \epsilon^{11 / 2}(\operatorname{tier}(\mathrm{ii})), \epsilon^{9 / 2}$ in turn and there is also an outer tier (iii) of normal extent $O\left(\epsilon^{9 / 2}\right)$ in $\bar{y}$. For the internal flow, I-III in step 2 are of order 1 (tier (i)), $R e^{-2 / 7}$ (tier (ii)), $R e^{1 / 7}$ in turn, after being as in $(2.3 d)$ previously, and the profile $u_{0}$ is assumed to satisfy the symmetry constraint in $(2.3 e)$. A wall sub-layer is also present as indicated.

the first-order equations

$$
\begin{gathered}
\tilde{u}_{X}+\tilde{v}_{y}=0, \\
\left(u_{0}-c\right) \tilde{u}_{X}+\tilde{v} u_{0}^{\prime}=-\tilde{p}_{X}, 0=-\tilde{p}_{y},
\end{gathered}
$$

while at second order we obtain the balances

$$
\begin{gathered}
\tilde{u}_{2 X}+\tilde{v}_{2 y}=0, \\
\left(u_{0}-c\right) \tilde{u}_{2 X}+\tilde{v}_{2} u_{0}^{\prime}+\left[\tilde{u}_{T}+\tilde{u} \tilde{u}_{X}+\tilde{v} \tilde{u}_{y}\right]=-\tilde{p}_{2 X}, \\
\theta_{1}\left[\left(u_{0}-c\right) \tilde{v}_{X}\right]=-\tilde{p}_{2 y} .
\end{gathered}
$$


The constant factor $\theta_{1}$ is zero for external flow and unity for internal flow. The solution of the leading-order system $(3.3 a-c)$ satisfying the required tangential-flow condition at $y=0+$ then has

$$
\tilde{v}=\tilde{p}_{X}\left(u_{0}(y)-c\right) f_{0}^{y}\left\{u_{0}(\hat{y})-c\right\}^{-2} \mathrm{~d} \hat{y},
$$

but with the scaled pressure response $\tilde{p}(X, T)$ remaining undetermined as yet. Instead the requirement of effectively zero displacement at large $y$ yields the break-up criterion (1.2) between the phase speed $c$ and the local streamwise velocity profile $u_{0}(y)$; this requirement, we note, follows from most of the interaction laws mentioned in $\S 2$, an exception being covered mainly by Brotherton-Ratcliffe $\&$ Smith (1987). The condition of effectively zero displacement here is supported by order-of-magnitude arguments, consistent with the law $(2.3 c)$ earlier (which implies that $A$ is of the typical order $\left|x-x_{0}\right||p|$ and so becomes negligible locally) and with the size of the displacement feedback in $(3.12 b)$ below for the external configuration, while we recall that the upper limit in (1.2) should read $a$ for the internal-flow configuration. The criterion (1.2) agrees with that in Smith (1988a) for step 1, as expected, and it is also related to the Burns criterion for water waves. The major novel feature for the current step 2, however, for internal flow, is the non-zero normal pressure gradient induced at second order via the inertial forcing term on the left-hand side of $(3.4 c)$. The other forcing term at second order, also shown in square brackets, is in (3.4b) and corresponds directly with the streamwise inertial effect found in Smith (1988a), leading to the $p_{1}$ equation in $\$ 2$ (and to part of (3.6) below). Solving (3.4a-c) then, given (3.5), (1.2), we obtain a compatability relation for the second-order terms which yields, after some manipulation, the governing equation between the unknown pressure response $\tilde{p}(X, T)$, the unknown jump contribution $j$ across the critical layer (tier (ii) below), and the effective displacement contribution $\Delta$ (from tier (iii) if present), namely

$$
a_{1} \tilde{p}_{T}+a_{2} \tilde{p} \tilde{p}_{X}=a_{3} \tilde{p}_{X X X}+j+\Delta .
$$

Here the constants $a_{1}, a_{2}\left(=\frac{1}{2} \hat{b}^{-1} a_{1}\right), a_{3}$ are integral properties of the local velocity profile, given by

$$
\begin{gathered}
a_{1} \equiv \tilde{p}^{-1} f_{0}^{\infty} \frac{\tilde{u}}{\left(u_{0}-c\right)^{2}} \mathrm{~d} y, \quad a_{2} \equiv \tilde{p}^{-2} f_{0}^{\infty} \frac{\left(\tilde{u}^{2}-\tilde{\psi} \tilde{u}^{\prime}\right) \mathrm{d} y}{\left(u_{0}-c\right)^{2}}, \\
a_{3} \equiv \tilde{p}^{-2} \theta_{1} f_{0}^{\infty} \tilde{\psi}^{2} \mathrm{~d} y, \quad \text { where } \tilde{\psi} \equiv \int_{0}^{y} \tilde{u} \mathrm{~d} y,
\end{gathered}
$$

with $a_{1}, a_{2}$ being essentially as in Smith (1988a) and $a_{3}$ is new, being associated with the new normal pressure-gradient influence for internal flow which results in the triple derivative in (3.6). The contribution $j$, due to a velocity-jump effect across the critical layer (ii), is analogous with that in Smith (1988a) for step 1 and it is considered next.

The second tier (ii), occurring near $y=y_{0}$, is a predominantly nonlinear critical layer for which the expansions

$$
\begin{gathered}
\bar{u}=\delta_{1}\left[c+\delta_{2}^{1 / 2} U_{1}+\delta_{2} U_{2}+\ldots+\delta_{2}^{2} U_{4}+\ldots\right], \\
\bar{\psi}=\delta_{1} \delta_{4}\left[\text { const. }+\delta_{2}^{1 / 2} c Y+\delta_{2} \Psi_{1}+\ldots\right], \\
\bar{p}=\delta_{1}^{2}\left[p_{0}+\delta_{2} P_{1}+\delta_{2}^{2} P_{2}+\delta_{2}^{5 / 2} P_{3}+\ldots\right],
\end{gathered}
$$


with

$$
\bar{y}=\delta_{4}\left(y_{0}+\delta_{2}^{1 / 2} Y\right)
$$

hold with $u_{0}\left(y_{0}\right)=c$ and the inflection-point condition $u_{0}^{\prime \prime}\left(y_{0}\right)=0$. Here $\bar{\psi}$ is the stream function satisfying $\bar{u}=\partial \bar{\psi} / \partial \bar{y}, \bar{v}=-\partial \bar{\psi} / \partial \bar{x}$. The form of $(3.7 a-d)$ is implied by the behaviour of the solutions in tier (i) as $y \rightarrow y_{0} \pm$. Substitution into the Navier-Stokes equations now produces the following streamwise-momentum balances:

$$
\begin{gathered}
U_{1} U_{1 X}-\Psi_{1 X} U_{1 Y}=-P_{1 X}, \\
U_{1 T}+U_{1} U_{2 X}+U_{2} U_{1 X}-\Psi_{1 X} U_{2 Y}-\Psi_{2 X} U_{1 Y}=0, \\
U_{2 T}+U_{1} U_{3 X}+U_{2} U_{2 X}+U_{3} U_{1 X}-\Psi_{1 X} U_{3 Y}-\Psi_{2 X} U_{2 Y}-\Psi_{3 X} U_{1 Y}=-P_{2 X}, \\
U_{3 T}+U_{1} U_{4 X}+U_{2} U_{3 X}+U_{3} U_{2 X}+U_{4} U_{1 X} \\
-\Psi_{1 X} U_{4 Y}-\Psi_{2 X} U_{3 Y}-\Psi_{3 X} U_{2 Y}-\Psi_{4 X} U_{1 Y}=-P_{3 X}+U_{1 Y Y},
\end{gathered}
$$

subject to appropriate matching conditions at large $|Y|$ analogous with those in Smith $(1988 a)$; and continuity requires that $U_{n}=\Psi_{n Y}(n=1,2, \ldots)$. The normal-momentum balance shows $P_{n Y}$ to be zero for $n<4$, so that $P_{1}=\tilde{p}, P_{2}=\tilde{p}_{2}\left(X, y_{0}, T\right)$, and so on. In $(3.8 a-d)$, the first equation shows the critical layer to be predominantly nonlinear, in the sense of Benney \& Bergeron (1969), Haberman (1972), the second and third equations turn out to be passive corrective effects, and the fourth equation provokes the velocity jump referred to in the previous paragraph. Thus the first few solutions are relatively straightforward,

$$
\begin{gathered}
\Psi_{1}=\frac{1}{2} b_{1} Y^{2}+b_{1}^{-1} \tilde{p}, U_{1}=b_{1} Y, V_{1}=-b_{1} \tilde{p}_{X}, \Psi_{2}=a_{11}(X, T) Y+b_{10}(X, T) \\
\Psi_{3}=\frac{1}{4} b_{3} Y^{4}+a_{12}(X, T) Y^{2}+b_{11}(X, T) Y+c_{10}(X, T) .
\end{gathered}
$$

Here $a_{11}=b_{1} I_{1} \tilde{p}$ where $I_{1}$ is the finite part of the integral in (3.5) evaluated at $y=y_{0}$, $b_{10}=b_{11}=0$ in the present context, $a_{12}=3 b_{3} b_{1}^{-2} \tilde{p}, c_{10 X}=b_{1}^{-1}\left(\tilde{p}_{2 X}+a_{11 T}+a_{11} a_{11 X}-\right.$ $\left.2 a_{12} a_{10 X}\right)$, the constants $b_{1}, b_{3}$ come from the local expansion $u_{0}-c=b_{1} s+b_{3} s^{3}+\ldots$ where $s \equiv\left(y-y_{0}\right)$, with the $b_{2}$-term absent due to the inflection point, and $V_{1} \equiv-\Psi_{1 X}$ is the scaled normal velocity. In contrast, the solution for $\Psi_{4}$ is more complicated. It can be written as $\Psi_{4}=\frac{1}{5} b_{4} Y^{5}+a_{13} Y^{3}+d_{10}+\Phi_{4}$ with $\Phi_{4} \sim c_{1 L} Y \ln |Y|+c_{11}{ }^{ \pm} Y$ at large $|Y|$, and then the $Y$-derivative of the streamwise momentum equation reduces to solving

$$
b_{1} Y \tau_{4 X}-b_{1}^{-1} \tilde{p}_{X} \tau_{4 Y}=-6 b_{1}^{-3}\left(b_{1} b_{3} \tilde{p}_{T}-2 b_{4} \tilde{p} \tilde{p}_{X}\right)\{\equiv m(X, T)\}
$$

for the scaled vorticity $\tau_{4} \equiv \Phi_{4 Y Y}$, subject to certain boundary conditions at large $|Y|$, from which the velocity jump can be found. The solution then follows from Smith \& Bodonyi (1987), giving the required velocity jump

$$
J=-b_{1}^{-1} f_{-\infty}^{\infty} m(s, T) \ln |\tilde{p}(X, T)-\tilde{p}(s, T)| \mathrm{d} s
$$

across the critical layer, in scaled terms, provided that $\tilde{p}$ remains monotonic in $X$. From this we can determine the jump term $j$, which is found to be $b_{1}^{-1} \partial J / \partial X$ and so can be expressed in terms of the unknown pressure $\tilde{p}$.

Tier (iii), which is present for the external configuration, lies outside tier (i) and has normal $\bar{y}$ extent comparable with the streamwise $\bar{x}$ extent, of order $\delta_{5}$. The flow solution is a small perturbation, of the uniform shear $\bar{u}=\epsilon^{-4} \bar{y}$, forced by the wall pressure response $\delta_{1}^{2} \delta_{2} \tilde{p}$ essentially (Savin 1996; Smith 1996), leading to Laplace's 
equation for the velocity perturbations. Hence a displacement effect is produced in the upper reaches of tier (i) associated with a $\partial \bar{v} / \partial \bar{y}$ variation of order $\delta_{1} \delta_{2} \delta_{4} / \delta_{5}^{2}$ and proportional to the Cauchy-Hilbert integral of $\partial^{2} \tilde{p} / \partial X^{2}$. This induced $\partial \tilde{v}_{2} / \partial \bar{y}$ is of the same order as that $\left(\delta_{1} \delta_{2}^{2} / \delta_{5}\right)$ corresponding to $\partial \tilde{v}_{2} / \partial y$ in $(3.2 b)$ since $\delta_{4}=\delta_{2} \delta_{5}$. In consequence

$$
\Delta=\frac{\theta_{2}}{\pi} f_{-\infty}^{\infty} \frac{\partial^{2} \tilde{p}}{\partial s^{2}}(s, T) \frac{\mathrm{d} s}{(X-s)}
$$

fixes the displacement (correction) effect, with $\theta_{2}$ being unity for the external context or zero for the internal case.

Combining the analyses of the two tiers (i), (ii), therefore, together with (iii) where necessary, we are left with the nonlinear evolution equation

$$
\begin{aligned}
a_{1} \tilde{p}_{T}+a_{2} \tilde{p} \tilde{p}_{X}=a_{3} \tilde{p}_{X X X}+\mu \tilde{p}_{X} f_{-\infty}^{\infty} \frac{\left[\tilde{p}_{T}(s, T)+a_{1}^{-1} a_{2}\left(\tilde{p} \tilde{p}_{s}\right)(s, T)\right] \mathrm{d} s}{\tilde{p}(X, T)-\tilde{p}(s, T)} \\
+\frac{\theta_{2}}{\pi} f_{-\infty}^{\infty} \tilde{p}_{s s}(s, T) \frac{\mathrm{d} s}{(X-s)}
\end{aligned}
$$

for the local scaled pressure $\tilde{p}(X, T)$, where $\mu=6 b_{3} b_{1}^{-4}$ is a positive or negative constant. We should repeat that an implicit property of the coefficient $\hat{b}$ (namely $\left.\hat{b}=a_{1} /\left(2 a_{2}\right)=-b_{1} b_{3} /\left(4 b_{4}\right)\right)$ is shown in Appendix A to hold true quite generally. The boundary conditions on $(3.12 a)$ are essentially

$$
\tilde{p} \propto|X|^{1 / 3} \text { as } X \rightarrow \pm \infty
$$

at finite times $T$, and the 'initial' conditions have the form

$$
\tilde{p} \sim|T|^{1 / 2} f(\xi) \text { as } T \rightarrow-\infty, \text { for } \xi \equiv X /|T|^{3 / 2} \text { of } O(1) .
$$

Here $(3.12 b)$ is to match the solution with that in the rest of the flow field, at $\bar{x}$ values outside the range of $(3.1 a)$, whereas $(3.12 c)$ is to join with the earlier behaviour in $(2.4 b)$ at the end of step 1 . Indeed, $\xi$ is effectively the same variable as in $(2.4 a)$, and $f(\xi) \equiv p_{1}(\xi)$; substitution of $(3.12 c)$ into (3.12a) leaves $p_{1}=f(\xi)$ controlled by the same equation as in $\S 2$, as required, and the constant $\hat{b}$ is verified as equalling $a_{1} /\left(2 a_{2}\right)$. See also (4.4) below. In (3.12a) the presence of the $\theta_{2}$ (or $\Delta$ ) term, when combined with the left-hand side alone, yields the Benjamin-Ono equation (cf. Kachanov, Ryzhov \& Smith 1993), an integro-differential equation which is consistent with a special case of Savin (1996), Smith (1996).

In the remainder of this section and in $\$ \$ 4-6$ we focus on the special internal-flow context of zero $\Delta$ (zero $\theta_{2}$ ) and unit $\theta_{1}$, i.e. the extended $\mathrm{KdV}$ equation applying for

$$
\theta_{1}=1, \quad \theta_{2}=0
$$

deferring further discussion of the extended Benjamin-Ono case corresponding to

$$
\theta_{1}=0, \quad \theta_{2}=1
$$

until §8. At large negative times $T$, then, the terms on the left-hand side of (3.12a) dominate and reproduce the break-up singularity of $\$ 2$, as required for matching. As scaled time $T$ then increases, the two remaining right-hand terms (with $\Delta$ neglected), due respectively to the normal pressure gradient and to the critical-layer jump, come progressively more into play. The latter, we note, happens to vanish identically when the leading-order form (3.12c) only is applied, at large negative times, cf. Smith (1988a) and $\S 2$. The appearance of the $\mathrm{KdV}$-like balance in (3.12a) is perhaps not so surprising 
in retrospect, since the $\mathrm{KdV}$ equation in its more familiar context of water waves arises from similar normal pressure-gradient effects, and likewise for the $\frac{1}{7}$ th powers in the scalings of (3.1), (3.2), cf. Whitham (1974), Smith (1977). It is worth observing that this structure does not hold in the severe type of break-up (see \$2) or in the reversed-flow singularity encountered for steady interacting boundary layers (Smith $1988 b$ ) where the full Euler equations are immediately encountered instead. Given the nonlinear $\mathrm{KdV}$-extended equation (3.12a) with (3.13), which generally requires a computational treatment as described below, it might be expected at first that, at large positive times $T$, the system produces travelling solitary waves and decaying waves. This is not true in general, however, as we shall see; the value of $\mu$ has an important influence.

\section{Solution behaviour for positive $\mu$}

The solution properties of $(3.12 a-c),(3.13)$ are found to depend crucially on whether the parameter $\mu$ is positive, negative or zero. In this section we consider positive values.

Computational solutions of $(3.12 a-c),(3.13)$ were obtained by the following approach. First, the initial condition $(3.12 c)$ was imposed at a large negative time $T=-\Gamma$ by transforming

$$
\tilde{p} \rightarrow a_{1} a_{2}^{-1} \Gamma^{1 / 2} \tilde{p}, \quad X \rightarrow \Gamma^{3 / 2} X, \quad T \rightarrow \Gamma T,
$$

which also serves to 'normalize' the constants present, converting $(3.12 a, c)$ to the form

$$
\begin{gathered}
\tilde{p}_{T}+\tilde{p} \tilde{p}_{X}=a_{4} \tilde{p}_{X X X}+\tilde{\mu} \tilde{p}_{X} \int_{-\infty}^{\infty} \frac{\left[\tilde{p}_{T}(s, T)+\tilde{p} \tilde{p}_{s}(s, T)\right] \mathrm{d} s}{\tilde{p}(X, T)-\tilde{p}(s, T)}, \\
\tilde{p} \sim|T|^{1 / 2} f(\xi) /(2 \hat{b}) \text { as } T \rightarrow-1,
\end{gathered}
$$

and leaving (3.12b) intact. Here $\tilde{\mu}=a_{1}^{-1} \mu$ is generally $O(1)$, as is $\hat{b}$, while $a_{4} \equiv$ $a_{3} a_{1}^{-1} \Gamma^{-7 / 2}$ is to be taken as small since $\Gamma$ is large and positive. Second, the starting behaviour (4.3) was imposed by setting

$$
f=-\frac{2}{\sqrt{3}} \sinh \left[\frac{1}{3} \sinh ^{-1}\left(\frac{3^{3 / 2} X}{2}\right)\right]
$$

which is an explicit solution for $p_{1}=f(\xi)$; see $\$ 2$ and also Hoyle (1991). Here we take the value $\hat{b}=\frac{1}{2}$ without loss of generality. Third, time-marching calculations of (4.2), (4.3) with $(3.12 b),(4.4)$ were performed using two methods. Preliminary computations by F.T.S. with Dr J. M. Hoyle reported in Hoyle (1991) tried the method of Greig \& Morris (1976) but modified to accommodate the unusual boundary condition (3.12b) and the principal-value integral in (4.2). For the boundary condition (3.12b), an appropriate simple moving solution of the inviscid Burgers equation was inserted at a few points near either end of the $X$-range of integration. The principal-value integral in (4.2), at any time step, was evaluated using a trapezoidal sum (similarly to Davis \& Werle 1982) with pressure values taken at the two time steps prior to the current computational one. Thus the integral was treated as a passive term in effect and this seemed to prove reasonably stable and accurate in some cases. The results were checked for grid-size effects, which were found to be small with a few exceptions; see next paragraph. Further, a change of sign in the coefficient $a_{4}$ can be accommodated by replacing $(\tilde{p}, X, \tilde{\mu})$ by $-(\tilde{p}, X, \tilde{\mu})$ in $(4.2),(4.3),(3.12 b)$, given also that $f$ in (4.4) is odd in $X$, and so $a_{4}$ can be taken as positive without loss of generality. 
This also demonstrates solution dependence on the parameter $\tilde{\mu} \operatorname{sgn}\left(a_{4}\right)$ generally, and especially the sign of $\tilde{\mu} a_{4}$; but $\tilde{\mu} a_{4}=\mu a_{3} a_{1}^{-2} \Gamma^{-7 / 2}$, with $a_{3}$ being positive from just below (3.6), and thus the sign dependence is essentially on the coefficient $\mu$ alone. Results were obtained for small positive values (typically 0.005 ) of the coefficient $a_{4}$, i.e. large $\Gamma$, and for various values of the coefficient $\tilde{\mu}$.

A more robust method was then adopted, which we now describe. We began this by adopting a fourth-order compact-difference scheme (Orszag \& Israeli 1974; Hirsh 1975; Aubert \& Deville 1983; Christie 1985; Li 1997) to compute the case of zero $\tilde{\mu}$, which is a standard $\mathrm{KdV}$ equation but with an unusual boundary condition in (3.12b), the latter being handled by asymptotic large- $|X|$ expansions to shorten the effective integration range in $|X|$. The computations showed the compact-difference scheme to be very suitable for that case. A fourth-order convergence rate was observed satisfactorily in refinement of the grid spacing and the results obtained were in agreement with previous ones. See $\mathrm{Li}$ (1997). Further discussion on the $\tilde{\mu}$ zero case is delayed to $\S 6$. For positive $\tilde{\mu}$ the compact-difference scheme was extended such that, at each time level, (4.2) is solved repeatedly in an iterative manner to force close satisfaction of (4.2) at that level, the principal-value integral (CI, say) being treated by a first-, second- or third-order formula developed using a Taylor expansion. The iterations applied here contrast with the passive way in which CI is treated in the previous method, and it is felt that the iterative procedure is central to guaranteeing that the resulting discrete system readily preserves the properties of the original continuous system as much as possible. Results are presented in figure $2(a-d)$, for various positive values of $\tilde{\mu}$. Tests on accuracy associated with the treatment of the CI term, with grid sizes, with iterative tolerance and with the effects of varying $\Gamma$, proved very affirmative indeed, as did tests on stability of the scheme. Again, the results agree broadly with ones obtained previously. For positive $\tilde{\mu}$ the numerical solutions tend to terminate at some finite time $T=\hat{T}_{0}$ at which the number of iterations exceeds a prescribed large number, say 100 . Generally, the larger $\tilde{\mu}$ is, the longer the solution lasts. At this termination time it was found that at some station $X=\hat{X}_{0}$ the negative gradient $-\partial \tilde{p} / \partial X\left(\hat{X}_{0}, \hat{T}_{0}\right)$ becomes very small, and around $\hat{X}_{0}$ the principal-value integral CI attains very large positive and/or negative amplitudes. (Here $\hat{X}_{0}, \hat{T}_{0}$ are identified with $X_{0}, T_{0}$ below in the limit of zero grid spacing). Again see figure $2(a-c)$. Also, the computations suggest that the location of $\hat{X}_{0}$ shifts in the negative $X$-direction, towards the origin, as $\tilde{\mu}$ increases, with the termination time increasing with $\tilde{\mu}$ : see figure $2(d)$.

The general behaviour of the computed solutions is as anticipated at the end of $\S 3$, with the left-hand terms in (4.2) dominating until the transformed time $T$ becomes relatively small. These terms force the pressure solution $\tilde{p}$ to move 'inward' until sufficiently large gradients are produced that the right-hand terms can counteract the inward trend. The special case of zero $\tilde{\mu}$ forms a canonical problem for the $\mathrm{KdV}$ equation in fact (see §6), and the solution can continue for all time $T$, forming a nonlinear modulated wave packet at large positive $T$. All the present cases however where $\tilde{\mu}$ is positive seem to terminate at the onset of a maximum/minimum in the scaled pressure $\tilde{p}$, at a finite time, in view of the final term in (4.2), as analysed below.

The response at the onset of a pressure maximum/minimum, at time $T=T_{0}-$ say, where

$$
\tilde{p}_{X} \rightarrow 0 \text { (at some station } X=X_{0} \text { ), }
$$

is significant for what follows later. Approximately, the scaled pressure response locally tends to be dominated by the triple derivative in (4.2), with $\tilde{p}$ then approximately 
$-d_{2}\left(X-X_{0}\right)^{3}+d_{3}\left(X-X_{0}\right)\left(T-T_{0}\right)$ to within an additive constant or so, where say the constants $d_{2}, d_{3}$ are both positive. In more detail, on the other hand, the local length scale around $X=X_{0}$ is therefore $O\left(T_{0}-T\right)^{1 / 2}$, so that $\chi \equiv\left(X-X_{0}\right) /\left(T_{0}-T\right)^{1 / 2}$ is $O(1)$, and

$$
\tilde{p} \sim d_{0}-d_{1}\left(T-T_{0}\right)+\left(T_{0}-T\right)^{3 / 2} g(\chi)+\ldots,
$$

where $d_{0}$ is the local pressure constant solution. From (4.2) the function $g(\chi)$ then satisfies

$$
-d_{1}=a_{4} g^{\prime \prime \prime}-d_{1} \tilde{\mu} g^{\prime} f_{-\infty}^{\infty} \frac{\mathrm{d} s}{g(\chi)-g(s)},
$$

subject to matching conditions with the pressure solution away from the turning point.

A suitably normalized form of (4.7), obtained by setting $\chi=\ell_{c} \chi_{c}, g=d_{1} \ell_{c}^{3} g_{c} /\left(6 a_{4}\right)$ where the positive length factor $\ell_{c}$ is unspecified as yet, is

$$
-6=g_{c}^{\prime \prime \prime}-6 \tilde{\mu} g_{c}^{\prime} f_{-\infty}^{\infty} \frac{\mathrm{d} s}{g_{c}(\chi)-g_{c}(s)} .
$$

The matching conditions then require

$$
g_{c}=-0\left(\chi_{c}^{3}\right) \text { at large }\left|\chi_{c}\right|
$$

in view of (4.6). The behaviour in $(4.8 a, b)$ may be made more specific as follows. If $(4.8 a)$ is considered iteratively say, with its final term regarded as being lagged, cf. below, then extra contributions $A_{0 c}+A_{1 c} \chi_{c}+A_{2 c} \chi_{c}^{2}$ are implied in $g_{c}$. At first sight the three constants here appear to be arbitrary, determined perhaps by solution properties of $\tilde{p}$ outside the $\chi$-zone. However, an origin shift in $\chi_{c}$, of $A_{2 c} / 3$, can be used to eliminate the extra $\chi_{c}^{2}$ contribution by virtue of the $\chi_{c}^{3}$ term in $(4.8 b)$ with unit coefficient in the iteration, or equivalently an integrated form of (4.8a) may be taken, while the constant contribution can then be absorbed into $g_{c}$. So only one arbitrary constant remains. Finally then the coefficient of the remaining $\chi_{c}$ contribution can be made equal to minus one by appropriate choice by $\ell_{c}$, given that the pressure is approaching a maximum/minimum as $T \rightarrow T_{0}-$. Hence we seek the solution which continues from $g_{c}=-\chi_{c}^{3}-\chi_{c}$ for zero $\tilde{\mu}$ and which in effect has zero $A_{0 c}, A_{2 c}$, with $-A_{1 c}$ kept as unity, for non-zero $\tilde{\mu}$. Another relevant point now arises from examining $(4.8 a, b)$ for small $\tilde{\mu}$, when $g_{c}=-\chi_{c}^{3}-\chi_{c}+6 \tilde{\mu} g_{c 1}$ say, yielding the linear equation

$$
g_{c 1}^{\prime \prime \prime}=\pi \sqrt{3}\left(\chi_{c}^{2}+4 / 3\right)^{-1 / 2} \chi_{c}
$$

for $g_{c 1}\left(\chi_{c}\right)$. Hence the values $g_{c 1}^{\prime \prime \prime}( \pm \infty)$ are different, implying that the $\chi_{c}^{3}$ coefficient associated with (4.8b) must be different at $\pm \infty$ for non-zero $\mu_{c}$. Also, we observe that integration gives a term $O\left(\chi_{c} \ell_{n}\left|\chi_{c}\right|\right)$ in $g_{c 1}$ at large $\left|\chi_{c}\right|$, which indicates a higher-order term $\propto\left|\chi_{c}\right|^{n}$ being present in $g_{c}$ as $\left|\chi_{c}\right| \rightarrow \infty$ with $n$ distinct from 1 when $\mu_{c}$ is non-zero. In consequence, a more precise rendition of $(4.8 b)$ is

$$
g_{c} \sim-D_{2}^{ \pm} \chi_{c}^{3} \text { as } \chi_{c} \rightarrow \pm \infty,
$$

where the constants $D_{2}^{ \pm}$are unequal in general, although both must be positive to maintain the sense of the terminal solution outside the $\chi$-zone and keep $g_{c}$ monotonic in $\chi_{c}$. In fact, analysis of $(4.8 a)$ at large positive $\chi_{c}$ subject to $(4.8 c)$ leads to the requirement

$$
D_{2}^{+}=1-\frac{\tilde{\mu} \pi}{\sqrt{3}}\left\{1+2\left(\frac{D_{2}^{+}}{D_{2}^{-}}\right)^{1 / 3}\right\}
$$


(a)
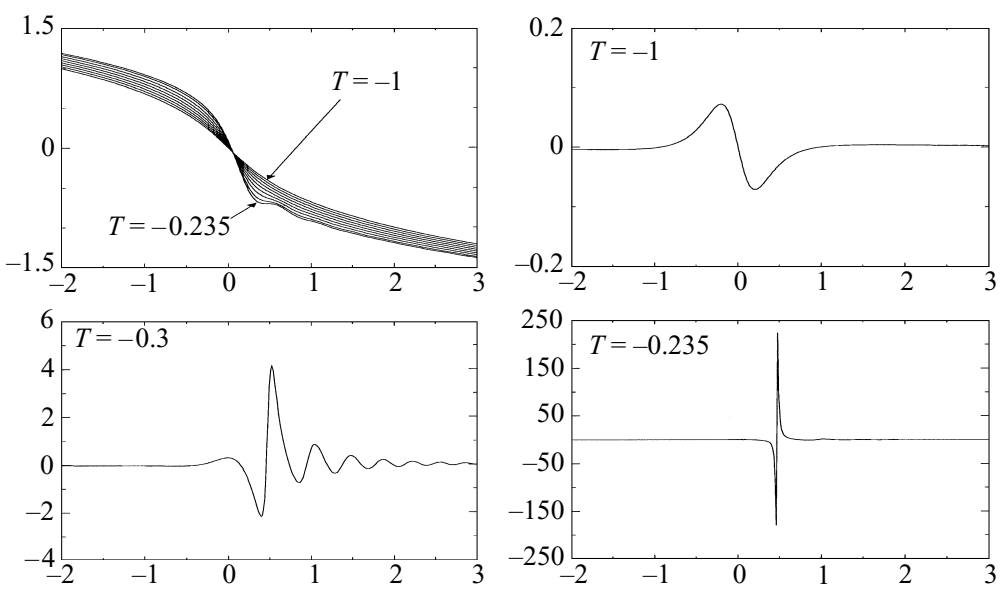

(b)
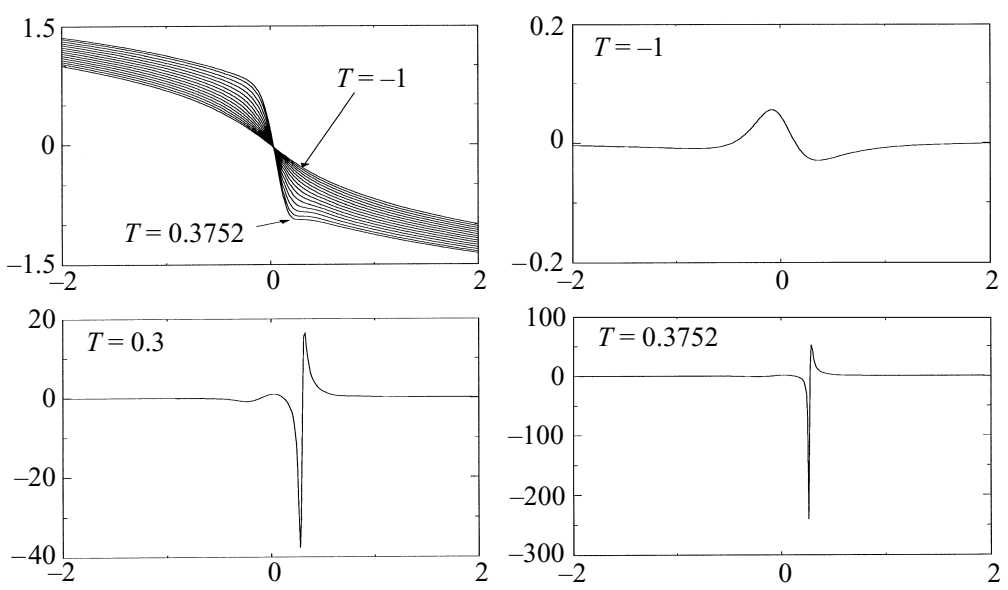

(c)
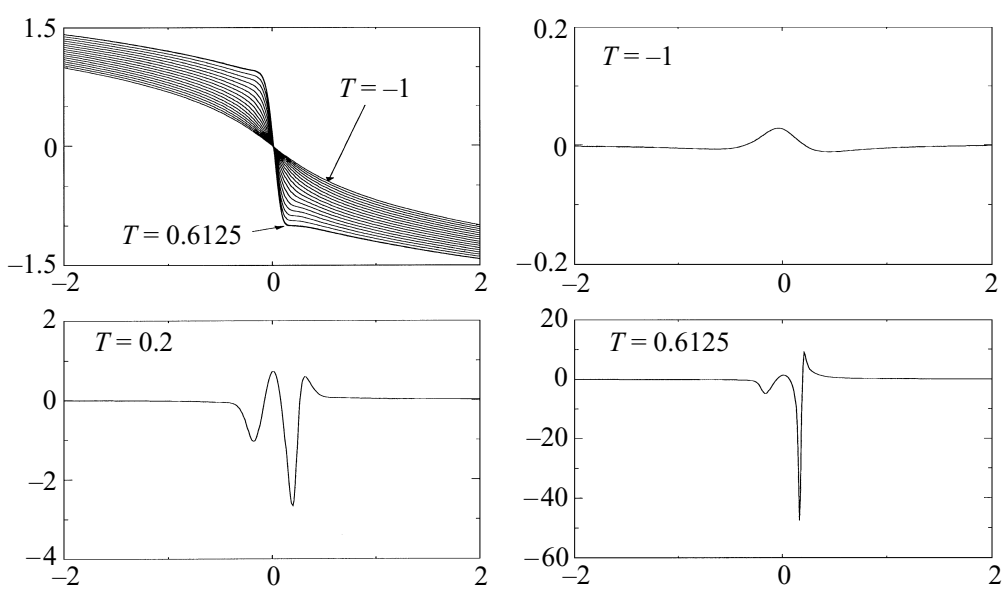

FIGURE $2(a-c)$. For caption see facing page. 
(d)
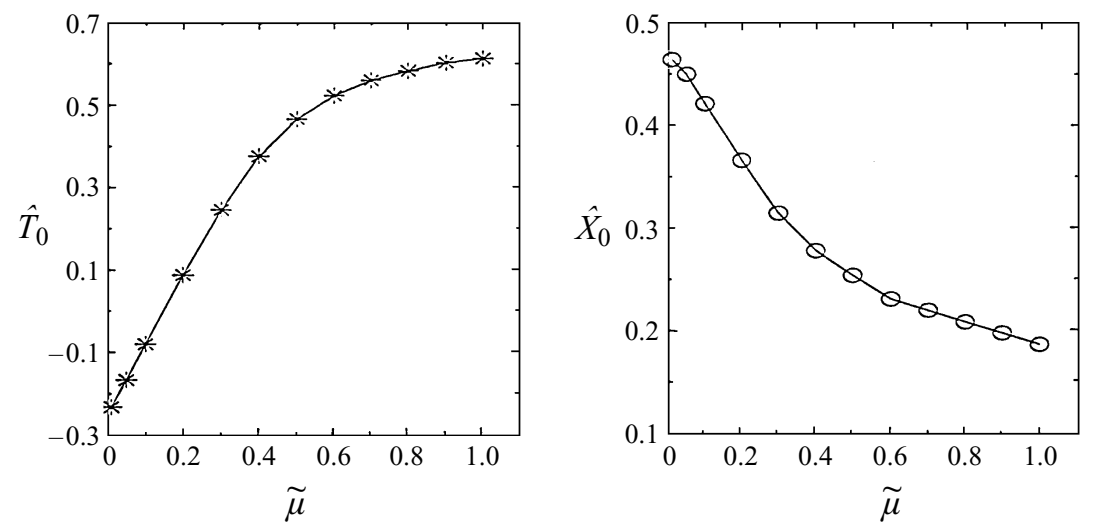

Figure 2. KdV-extended computed solutions, for $\tilde{\mu}$ and $a_{4}$ positive, in (4.2). See $\S 4$. Here $(a-c)$ are for $\tilde{\mu}=0.01,0.4,1$ in turn, showing $\tilde{p}$ along with the principal-value integral CI at three times in each case, while $(d)$ gives the termination position and time against $\tilde{\mu}$.

and similarly the large negative range requires that

$$
D_{2}^{-}=1+\frac{\tilde{\mu} \pi}{\sqrt{3}}\left\{1+2\left(\frac{D_{2}^{-}}{D_{2}^{+}}\right)^{1 / 3}\right\} .
$$

It follows that the ratio $D \equiv D_{2}^{+} / D_{2}^{-}$, which must be positive, is fixed by the relation $\left(1-D^{1 / 3}\right) /\left(1+D^{1 / 3}\right)=\tilde{\mu} \pi /(\sqrt{3})$ for a given $\tilde{\mu}$. Hence

$$
D=(1-\tilde{\mu} \pi / \sqrt{3})^{3} /(1+\tilde{\mu} \pi / \sqrt{3})^{3}
$$

determines $D$ uniquely for the interval $|\tilde{\mu}|<\sqrt{3} / \pi$ as shown in figure $3(a)$ : see also comments in $\S 8$. Then the values of $D_{2}^{ \pm}$can be found from $(4.9 a$ or $b)$.

Numerical solutions of $(4.8 a, c)$ with $D_{2}^{ \pm}$determined via $(4.9 a-c)$ were obtained for a number of $\tilde{\mu}$ values, taking into account the specification of the three constants $A_{0 c}, A_{1 c}, A_{2 c}$ described after (4.8b). A finite-difference approach of nominal secondorder accuracy was followed, for given $D$ and hence $\tilde{\mu}$, setting $g_{c}^{\prime}=q$ and coupling this equation with that derived by replacing $g_{c}^{\prime \prime \prime}$ in $(4.8 a)$ by $q^{\prime \prime}$. Two-point differencing was used on the $g_{c}^{\prime}$ equation and centred differencing on the $q^{\prime \prime}$ one, over a range $\chi_{1} \leqslant \chi_{c} \leqslant \chi_{2}$, with the principal value integral evaluated as a summation as in Davis \& Werle (1982) but including contributions from $3 \chi_{1}$ to $\chi_{1}$ and $\chi_{2}$ to $3 \chi_{2}$. Concerning the boundary conditions and allowing for the three constants above, the values $-D_{2}^{+} \chi_{c}^{3}-\chi_{c}$ were imposed on $g_{c}$ at the most left- and right-hand grid points $\chi_{1}(<0), \chi_{2}(>0)$ respectively, along with the value $-3 D_{2}^{-} \chi_{1}^{2}-1$ at the former point to fix the solution. Newton iteration was then applied to the difference equations but with lagging used on all the summation terms except for the diagonal elements in the matrix inversion required. In some cases under-relaxation was also adopted when the iterative increments were added to the previous guesses. The iterative process was continued until the solution was sufficiently converged. With a typical grid of $-4(0.02) 4$ in $\chi_{c}$ symmetric about the origin approximately 2000 iterations were required to reduce the increments to below $10^{-5}$, depending on the value of $\tilde{\mu}$. The results were also checked by other methods including an artificial-time marching one to a steady state, whose solutions agreed closely with the present solutions. The equation $(4.8 a)$ was found to be satisfied to within a residue of less than 0.0005 

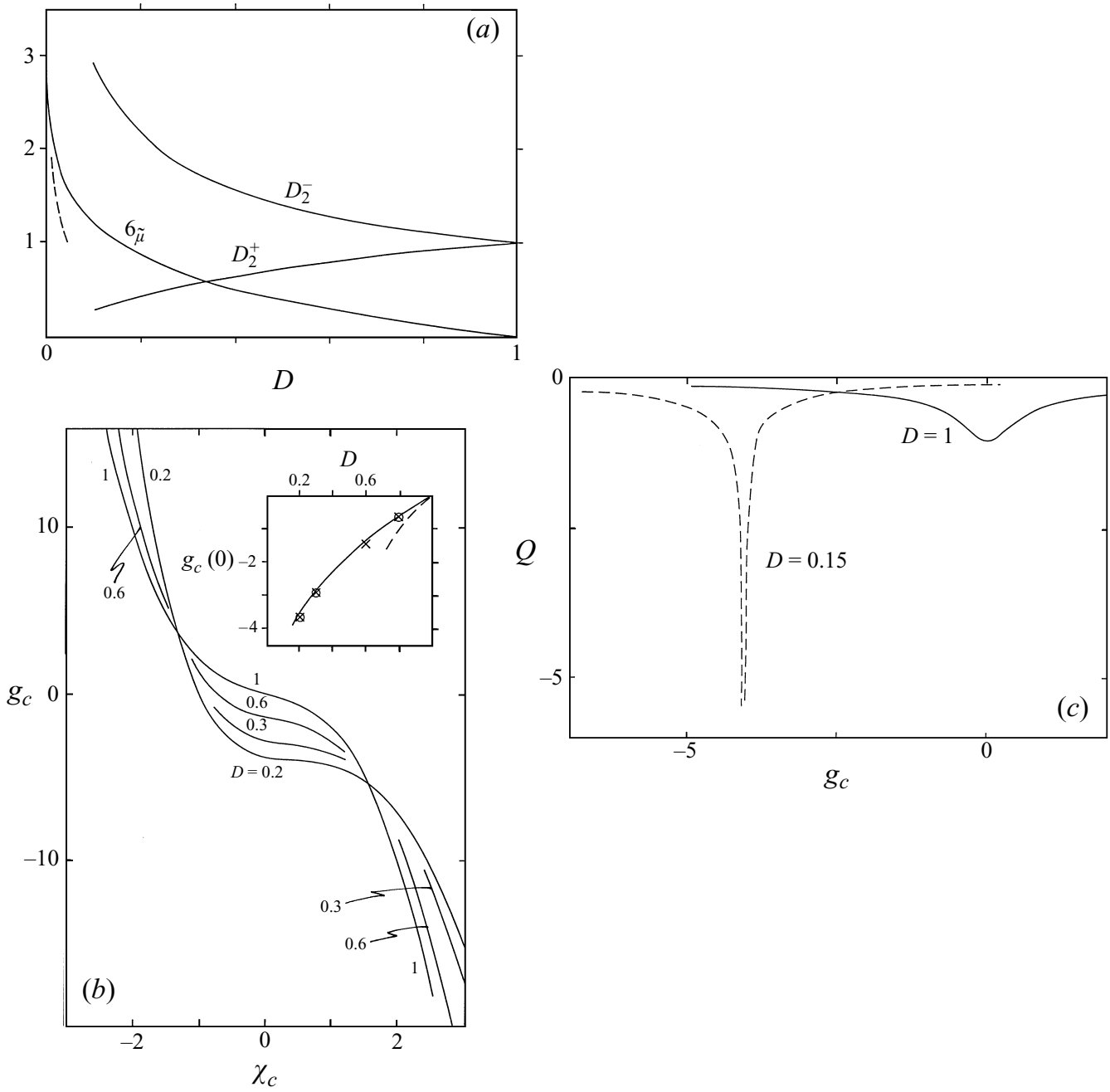

Figure 3. (a) Dependence of $\tilde{\mu}, D_{2}^{ \pm}$on $D$. Dashes show asymptote as $D \rightarrow 0+, \tilde{\mu} \rightarrow \sqrt{3} / \pi(\approx 0.5513)$. (b) Sample results for $g_{c}$ versus $\chi_{c}$, for $D=0.2,0.3,0.6,1$. The inset shows the variation of $g_{c}(0)$ with $D$, together with small- $(D-1)($ small- $\tilde{\mu})$ theory (dashed curve) and grid effects $(0, \times)$. The boundary here has $\left|\chi_{c}\right|=4$. Similar trends result for a boundary at $\left|\chi_{c}\right|=8$. (c) $Q$ versus $g_{c}$, where $Q \equiv 1 /\left(\mathrm{d} g_{c} / \mathrm{d} \chi_{c}\right)$ : see Appendix B.

compared with a typical maximum term of magnitude $O\left(10^{3}\right)$. Sample results are shown in figure $3(b)$ for positive $\tilde{\mu}$. Solutions for negative $\tilde{\mu}$ values may also be obtained however: simply reverse the signs of $g_{c}, \chi_{c}, \tilde{\mu}$ above, and interchange $D_{2}^{ \pm}$, in $(4.8 a-c)$ and the figure. Similarly the graph of $\tilde{\mu}$ versus $D$ for negative $\tilde{\mu}$ may be derived by replacing $(\tilde{\mu}, D)$ in figure $3(a)$ by $\left(-\tilde{\mu}, D^{-1}\right)$, this leading to $\tilde{\mu} \rightarrow-\sqrt{3} / \pi$ as $D \rightarrow \infty$. Further aspects of the terminal solutions are discussed briefly in Appendix B.

The extended KdV computations in figure 2 seem to fall in line with the local terminal analysis in (4.6)-(4.9), for a range of non-zero $\tilde{\mu}$ values. The growth in magnitude of the principal-value integral CI in figure 2 near the end of the computations for instance is in line with the large order of magnitude $\mathrm{CI}=O\left(T_{0}-T\right)^{-1}$ predicted by the terminal analysis.

New physics is bound to come into operation next, associated primarily with a change in the nonlinear critical-layer dynamics as studied in $\$ 7$. Hence the present 
flow structure breaks down locally, marking the end of step 2 before the regime of soliton formation mentioned earlier can be reached (directly anyway, cf. §6). We move on to address the case of $\mu<0$.

\section{Negative $\mu$}

Computations of (4.2)-(4.4) with (3.12b) but for $\tilde{\mu}$ negative with $a_{4}$ positive (corresponding to negative $\mu$ ) were performed using the second numerical method described in the previous section. The results are summarized in figure $4(a, b)$ for $\tilde{\mu}=-0.2$, which gives the fairly characteristic response for negative $\tilde{\mu}$ values; others are in $\mathrm{Li}$ (1997). Contrary to what might be expected from (4.6ff), the results for $\tilde{\mu}<0$ indicate a new feature appearing, that of short-scale instability. Weak approximately wavelike disturbances are found to grow fast temporally and their typical growth rates increase with refinement of the grid. This negative $\tilde{\mu}$ case is a more complicated one numerically, needing more effort on the modified computational method. Different grid spacings and time steps showed the numerical solutions to be unstable, generally terminating at significantly different times for different space and time steps, with smaller steps yielding earlier terminations; the termination times are also somewhat scheme-dependent. The form of this numerical phenomenon suggests, then, the presence of a short-wave instability for $\tilde{\mu}$ negative (cf. Moore 1979; Smith 1982; Ryzhov \& Smith 1984 in other settings).

An analytical explanation seems to be the following. We focus on short waves of typical given wavenumber $\alpha \gg 1$ and unknown (complex) frequency $\omega$. Then for a given base solution $\tilde{p}_{0}(X, T)$ say a linear disturbance of small amplitude $O(\hat{\varepsilon})$ in $\tilde{p}$ and proportional to $\exp (\mathrm{i} \alpha X-\mathrm{i} \omega T)$ satisfies for positive $\alpha$ the eigenrelation

$$
-\mathrm{i} \omega+\tilde{p}_{0} \mathrm{i} \alpha=-\mathrm{i} \alpha^{3} a_{4}-\tilde{\mu}\left(-\mathrm{i} \omega+\tilde{p}_{0} \mathrm{i} \alpha\right) \mathrm{i} \pi
$$

from (4.2), since the $X, T$ variation in $\tilde{p}_{0}$ is negligible now as in a parallel-flow approximation. The contribution $\propto \mathrm{i} \pi$ in (5.1) stems from the Cauchy integral of $\exp (\mathrm{i} \alpha \tilde{x}) /\left((X-\tilde{x}) p_{0}^{\prime}\right)$ with respect to $\tilde{x}$, with $p_{0}^{\prime}$ denoting $\partial \tilde{p} / \partial X(X, T)$ which here is constant to leading order. From (5.1) we then have the complex frequency

$$
\omega=\alpha \tilde{p}_{0}-i \alpha^{3} a_{4}(\tilde{\mu} \pi+\mathrm{i})\left(\tilde{\mu}^{2} \pi^{2}+1\right)^{-1},
$$

where strictly the $\alpha \tilde{p}_{0}$ term could be omitted at first order. In any case the implied temporal growth rate is

$$
\omega_{i}=\frac{-\tilde{\mu} \pi \alpha^{3} a_{4}}{\left(\tilde{\mu}^{2} \pi^{2}+1\right)} .
$$

Hence there is short-scale instability, with positive $\omega_{i}$, for any negative $\tilde{\mu}$; more precisely this is for negative $\tilde{\mu} a_{4}$, corresponding to negative $\mu$, although here we mostly take $a_{4}$ as positive anyway. We should remark that the terms on the right-hand side of (4.2) are responsible for the result (5.3) and that (5.1)-(5.3) can be extended to a Fourier transform result in an initial-value setting if necessary. Moreover, the growth rate $\omega_{i}$ increases very rapidly like $\alpha^{3}$ for increasing wavenumbers $\alpha$, thus probably making the present nonlinear initial-value problem ill-posed for negative $\tilde{\mu}$, although the linear result (5.3) remains valid only for $\hat{\varepsilon} \alpha \ll 1$.

The computations in figure 4 are qualitatively in keeping with the above analysis. Attempts to obtain quantitative agreement were also made and were partly encouraging, although the very nature of the explosive secondary instability according to the analysis is difficult to handle with sufficient accuracy numerically. Resolution is a 
(a)
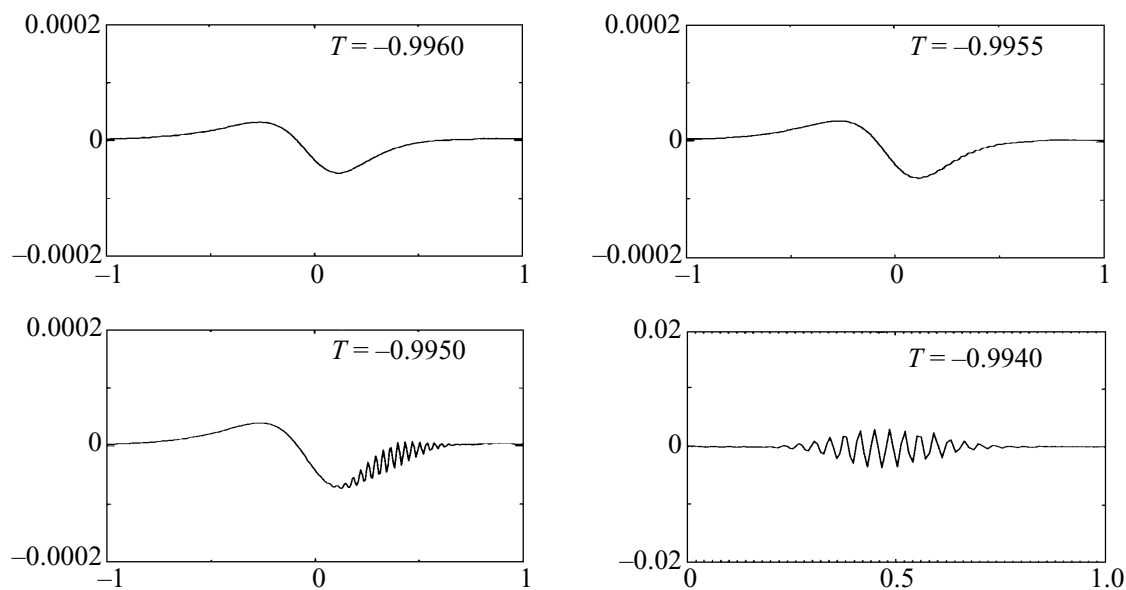

(b)

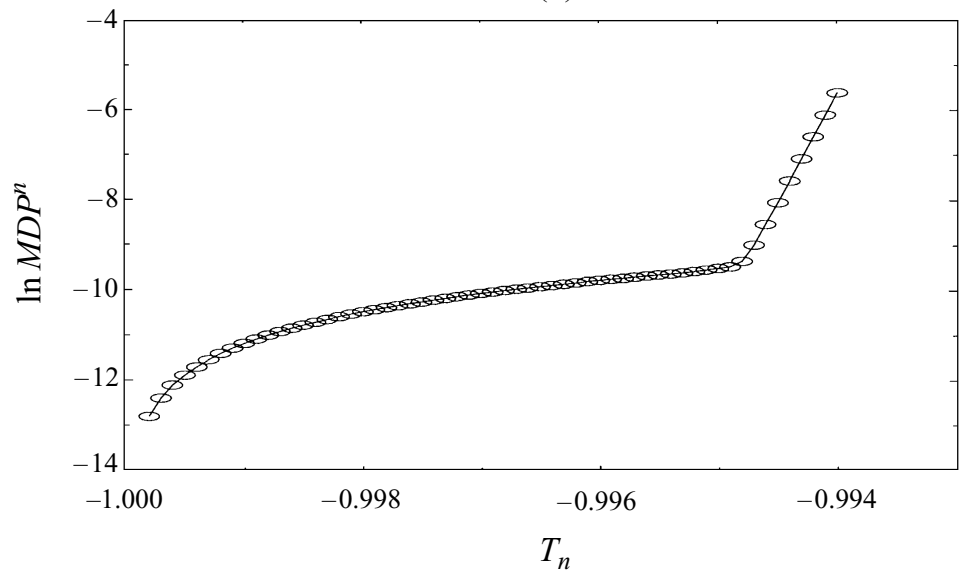

Figure 4. Computed results for negative $\tilde{\mu}$, positive $a_{4}$ in (4.2): see $\$ 5$. (a) $\Delta \tilde{p}(X, T) \equiv \tilde{p}(\tilde{\mu}=-0.2)-\tilde{p}(\tilde{\mu}=0)$ versus $X$; here $\Delta T=0.0001, h=0.006, a_{4}=0.005 .(b)$ $\ln \left(M D P^{n}\right)$ versus $T ; \Delta T, h, a_{4}$ are as in $(a)$.

key factor, requiring a very small grid spacing, but that can enlarge the computation time excessively. In our further work we turned to a coordinate transformation to cluster grid points into the region of high activity in the solution (Li 1997), adopting a second-order method coupled with a second-order formula for the principal-value integral CI to compute in the transformed plane. This enabled much more resolution to be applied generally. The subsequent results agreed with the limiting analysis and in particular (5.3) to within a factor of about 2 in the temporal growth rate derived from a $\log$ plot of successive numerical results, for a wavenumber $\alpha$ estimated to be in the range 100-200, even though near the end of the calculations the typical value of $\hat{\epsilon} \alpha$ rose to more than unity, indicating extra nonlinear effects, cf. the end of the previous paragraph.

More details on the numerical growth rate are as follows. To evaluate it a base solution (say $\tilde{P}_{0}$ ) is needed for comparison, a seemingly reasonable choice being the 
(stable) solution for zero $\tilde{\mu}$ since the instability for negative $\tilde{\mu}$ develops soon after the start of the calculation. Thus, after each time step, the maximum difference denoted by $M D P^{n}\left(=\max _{j}\left|\tilde{p}\left(X_{j}, T_{n}\right)_{\tilde{\mu}<0}-\tilde{p}\left(X_{j}, T_{n}\right)_{\tilde{\mu}=0}\right|\right)$ between the results for negative $\tilde{\mu}$ and those for zero $\tilde{\mu}$ is treated as the amplitude of the disturbances. Figure 4(a) shows the evolution of $\Delta \tilde{p}\left(X_{j}, T_{n}\right)=\tilde{p}\left(X_{j}, T_{n}\right)_{\tilde{\mu}=-0.2}-\tilde{p}\left(X_{j}, T_{n}\right)_{\tilde{\mu}=0}$ with time-marching, where the short waves of the disturbance become more evident. The disturbance wavenumber $\alpha$ for the evaluation of $\omega_{i}$ is approximated by estimating the wavelength $\lambda(=2 \pi / \alpha)$ of the short waves. For the above case the estimated wavelength is 0.0365 , so that $\alpha \approx 172$, and $\omega_{i} \approx 11490$ from (5.3). It is found that once the disturbance emerges clearly its wavelength does not change throughout the late stages of computation for all cases we computed. The estimate of the numerical growth rates $\omega_{n}$ is based on the calculation of the maximum difference $M D P^{n}$. At first $M D P^{n}$ are centred near $X=0$ and grow only slowly, but at a certain time they start to grow fast at a location different from the original one, apparently in an exponential manner. The logarithm, $\ln M D P^{n}$, should approach $-\omega_{i} T$ according to the analysis in (5.1)-(5.3), for asymptotically short wavelengths in the disturbance, and indeed the numerical results do show a nearly linear development with $T$ near the end of the calculation; see figure $4(b)$. Therefore the numerical growth rates can be evaluated by calculating the slope of the curve of $\ln M D P^{n}$ versus $T$. The values of $\omega_{n}$ for the above case are estimated to be around 5000 in the last 7-8 steps. This is a typical case. Several other results for different $\tilde{\mu}, a_{4}$, with $\tilde{\mu} a_{4}$ negative, as well as for different steps $\Delta T$ and grid spacings $h$ (in the transformed plane), are displayed by Li (1997), and here we summarize the computational results as follows. First, for fixed $h, \tilde{\mu}$ and $a_{4}$, decreasing the time step $\Delta T$ leads to decreasing $\lambda$ and increasing numerical growth rate $\omega_{n}$, in keeping with (5.3). Second, for fixed $\Delta T, h$ and $a_{4}$, increasing $|\tilde{\mu}|(<1)$ leads to a slight change in $\lambda$ (this change is possibly due to numerical accuracy) but increasing numerical growth rate $\omega_{n}$, again in keeping with (5.3). Third, for fixed $\Delta T, h$ and $\tilde{\mu}$, increasing $a_{4}$ leads to significantly increasing $\lambda$, but the change in the numerical growth rate $\omega_{n}$ is small. This is also in keeping with (5.3). Fourth, for fixed $\Delta T, \tilde{\mu}$ and $a_{4}$, a change in $h$ leads to a slight change in $\lambda$ and the numerical growth rate $\omega_{n}$, and it seems the change is due to numerical inaccuracy. Fifth, quantitatively, most numerical growth rates produced fall into a range of values of approximately half the theoretical growth rates in the last stage of calculations, although this is quite sensitive to the value of $\alpha$ used in (5.3).

The secondary instability here implies that an examinaton of much shorter time and space scales (as in the references mentioned earlier in this section and more recently, in a different setting, Davis 1992; Ryzhov \& Terent'ev 1997) is probably necessary prior to the current step. It is interesting that the instability and illposedness are based mainly on the balance of the new normal pressure gradient and the nonlinear critical-layer jump arising in this work. It might be argued that after a fast-growing disturbance such as in (5.1)-(5.3) becomes nonlinear a finite-time singularity is encountered just as in $\S 4$. The more satisfying conclusion however is as above, that extra physical effects need to be brought into operation beforehand, quite possibly associated with a streamwise length scale equal or closer to $O\left(\epsilon^{5}\right)$ rather than the longer scale of the current step 2.

\section{Zero $\mu$}

For zero $\tilde{\mu}$ (and hence zero $\mu$ ) (4.2) becomes a standard KdV equation, of which many solution properties are well known, e.g. Drazin (1983), Drazin \& Johnson 
(1989), Miles (1981), Whitham (1974). The boundary conditions (4.3), (4.4), (3.12b) on the other hand appear to be non-standard, although related to step-like initial conditions (see Peregrine 1966; Gurevich \& Pitaevskii 1974), and they are found to provoke an interesting response in the solution, which, unlike those of $\S \S 4,5$, continues to large positive times.

Computational results for (4.2)-(4.4), (3.12b) with $\tilde{\mu}$ zero, derived as in the previous two sections, are given in figure 5 at times $T$ from -1 to 9 . Following the smooth start at large negative time corresponding to $T=-1$, the solution gradually develops multiple (smooth) maxima and minima with increasing $T$ and as expected multiple waves and possible solitons appear at large positive $T$. The accuracy and stability of the compact-difference method used was also checked against other methods for other $\mathrm{KdV}$ cases, yielding favourable comparisons. In figure 5 at larger times a beautiful wave packet travels along in the positive $X$-direction, and numerically it seems this travel can be maintained accurately for a very long time indeed provided that sufficiently small grid spacings and time steps are taken.

Of concern next is the solution behaviour as time $T \rightarrow \infty$. An analysis of this based on Whitham's (1974) approach (and figure 6(a)) is presented in Appendix C. Figure 6(b) shows the computational results for the amplitude of the first soliton and its centre position as a function of time $T$, together with the asymptotic large-time curves given by the analysis of Appendix C. The agreement is good for the amplitude, whereas the centre-position prediction is more sensitive to the value of a coefficient (Appendix $\mathrm{C}$ ) in the asymptote as the example in the figure indicates.

\section{Vortex wind-up: step 3}

From $\S 4$, as time $T$ approaches $T_{0}-$ when the scaled pressure first develops a local maximum or minimum spatially, the unsteady forces in the critical layer become significant relative to the nonlinear ones (cf. Dickinson 1970; Haberman 1972; Brown \& Stewartson 1978; Hickernell 1984; Bodonyi, Smith \& Gajjar 1983; Gajjar \& Smith 1985; Haynes \& Cowley 1986; Smith \& Bodonyi 1987). For the balance of forces then leaves the typical streamwise and normal length scales of the nonlinear critical layer decreasing as $\left(T_{0}-T\right)^{1 / 2},\left(T_{0}-T\right)^{3 / 4}$ respectively, in a similarity form. Hence the main inertial operator there, which is spatial and proportional to $|\bar{y}| \partial / \partial \bar{x}$ (scaled), diminishes as $\left(T_{0}-T\right)^{1 / 4}$ whereas the temporal operator is of relative order $\phi\left(T_{0}-T\right)^{-1}$, lagging behind the spatial operators in step 2 by an amount $\phi \equiv \epsilon^{2 / 7}$. The new balance of concern here therefore occurs when $T_{0}-T$ is as small as $\phi^{4 / 5}$. That makes the characteristic $\bar{x}, \bar{y}$ scales decrease by factors $\phi^{2 / 5}, \phi^{3 / 5}$, from above.

The orders of magnitude, then, point to a new basic process, step 3, which is that of local vortex formation arising inside the nonlinear unsteady critical layer, whereas outside the flow structure remains much as in step 2. The length and time scales in the appropriate moving frame are reduced in step 3 , by respective factors $\epsilon^{4 / 35}, \epsilon^{8 / 35}$ compared with step 2, so that

$$
\begin{gathered}
\bar{x}=\text { constant }+\phi^{153 / 10}\left\{c \tilde{t}+\phi^{8 / 5} \tilde{x}\right\}, \\
\bar{t}=\text { constant }+\phi^{118 / 10} \tilde{t},
\end{gathered}
$$

while the velocity and pressure fields are given by

$$
\begin{aligned}
\bar{u}=\epsilon c+\phi^{51 / 10} b_{1} \tilde{y} & +\ldots+\phi^{67 / 10} I_{1} b_{1} P_{2}+\ldots+\phi^{71 / 10}\left(6 b_{3} b_{1}^{-2} d_{0} \tilde{y}\right) \\
& +\phi^{75 / 10} I_{1} b_{1} P_{3}+\phi^{79 / 10} \tilde{u}_{0}+\phi^{83 / 10}(\tilde{u}+\tilde{\gamma} \ln \phi)+\ldots,
\end{aligned}
$$




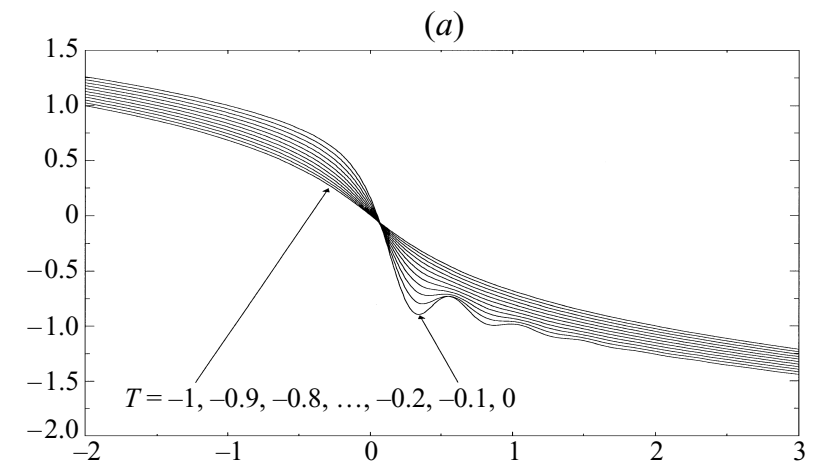

(b)
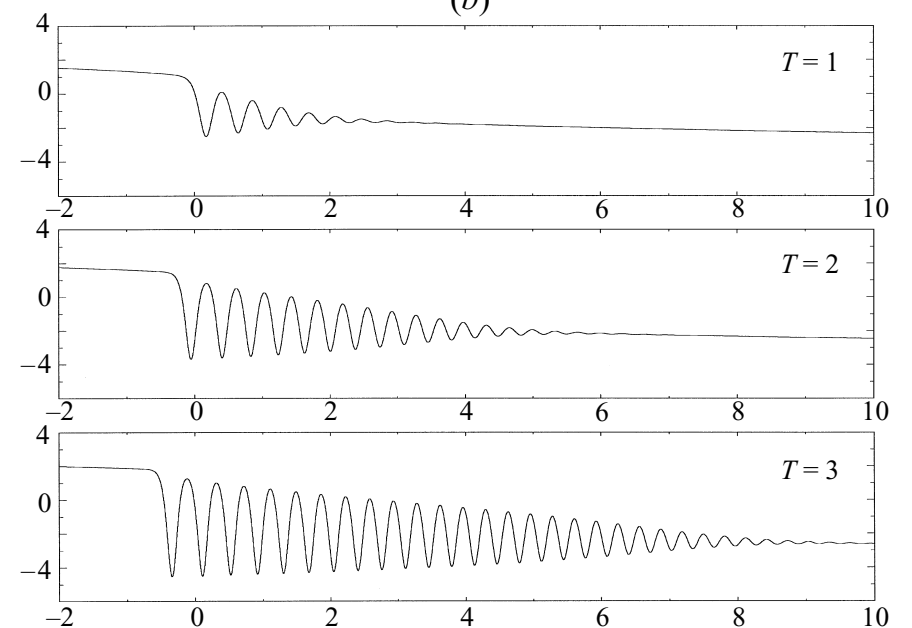

(c)

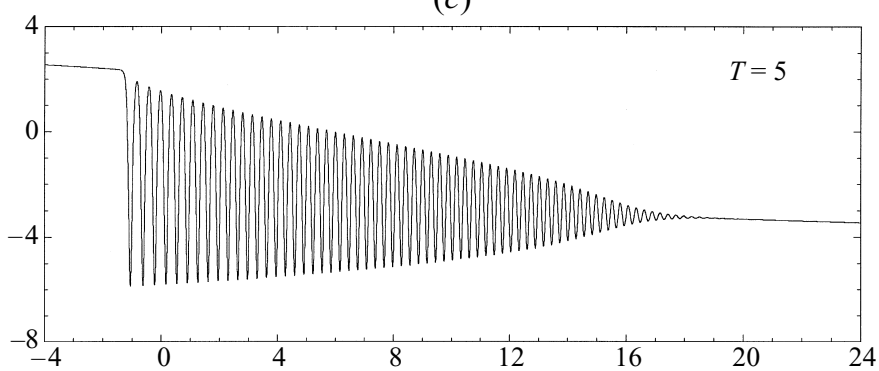

(d)

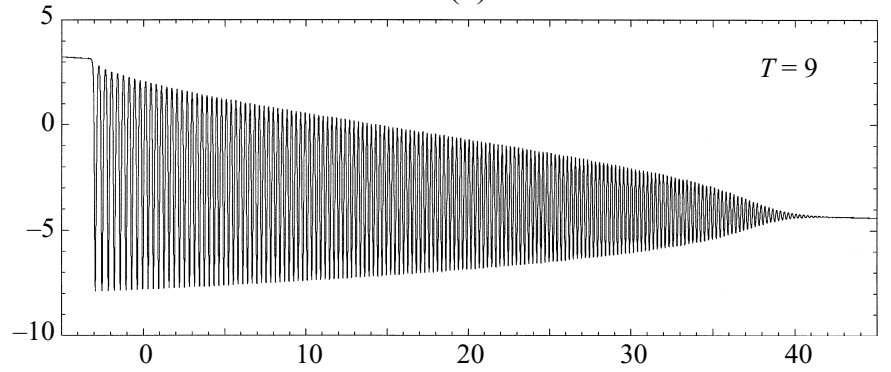

Figure 5. Computed results for zero $\tilde{\mu}$ in $(4.2)$ : see $\S 6$. Here $(a-d)$ give the pressure $\tilde{p}$ versus $X$ at times $T$ varying from -1 to 9 . We observe incidentally that the time and location of the first minimum in $\tilde{p}$ correspond to the limiting values of $\hat{T}_{0}, \hat{X}_{0}$ as $\tilde{\mu} \rightarrow 0+$ in figure $2(d)$. 
(a)

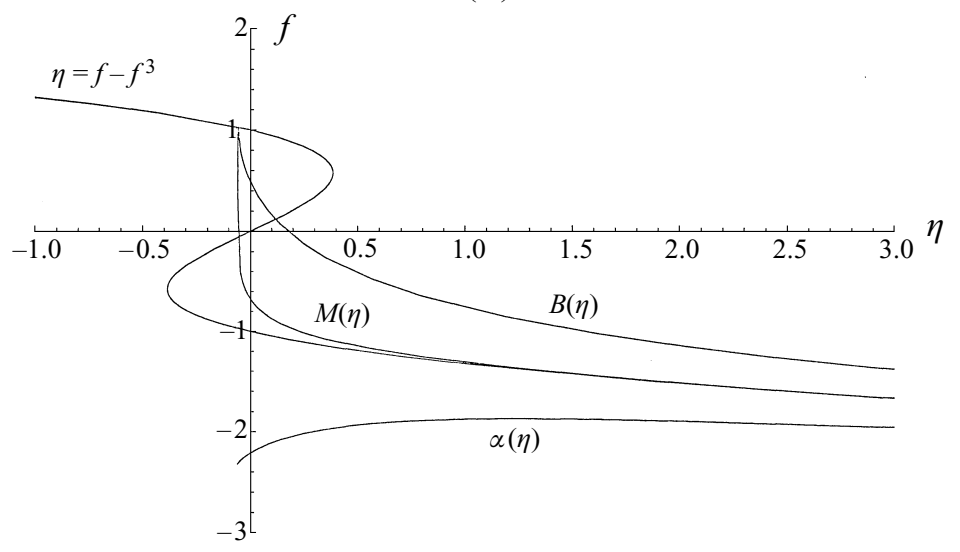

(b)

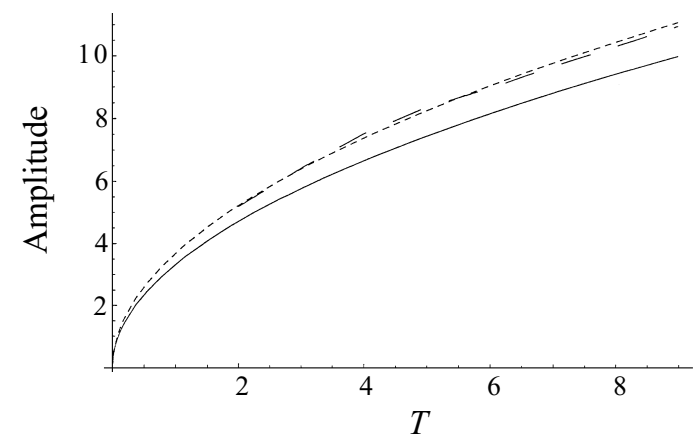

$T$

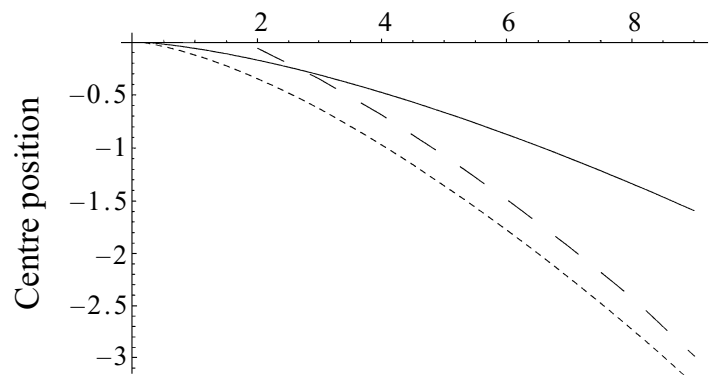

FIGURE 6. (a) The structure of the zero- $\tilde{\mu}$ solution as $T \rightarrow \infty$. For $\eta<\eta_{s}$, the solution is given by the curve $\eta=f-f^{3}$ with $\tilde{p}=T^{1 / 2} f(\eta), \eta=X / T^{3 / 2}$. For $\eta>\eta_{s}$, the solution has the form of a wave packet of cnoidal waves, with envelope $\beta \geqslant f \geqslant a$ and mean $M(\eta)$. The mean part is continuous at $\eta=\eta_{s}$. (b) Comparisons between large-time predictions (solid curve) and computational results (long dashes), for times $T$ up to 9 . See $\$ 6$ and Appendix C. The short-dashed curve shows predictions taking $\eta_{s}=-0.12$, rather than -0.0583 as the theory predicts.

$$
\begin{gathered}
\bar{v}=- \\
\phi^{73 / 10} P_{2 \tilde{x}} b_{1}^{-1}+\ldots-\phi^{81 / 10} P_{3 \tilde{x}} b_{1}^{-1}-\phi^{89 / 10}\left\{b_{1} \tilde{y} P_{2 \tilde{x}}+P_{2 \tilde{t}}\right\} I_{1}-\phi^{91 / 10} P_{4 \tilde{x}} b_{1}^{-1} \\
-\phi^{97 / 10}\left\{b_{1} \tilde{y} P_{3 \tilde{x}}+P_{3 \tilde{t}\}} I_{1}+\phi^{101 / 10} \tilde{v}_{0}-\phi^{101 / 10} P_{5 \tilde{x}} b_{1}^{-1}+\phi^{21 / 2} \tilde{v}+\ldots\right. \\
\bar{p}=\epsilon^{2} p_{0}+\phi^{9} d_{0}-\phi^{98 / 10} d_{1} \tilde{t}+\phi^{102 / 10} P_{2}+\ldots+\phi^{11} P_{3}+\phi^{12} P_{4} \ldots
\end{gathered}
$$

in the critical layer where $\bar{y}=\epsilon^{5}\left(y_{0}+\phi^{8 / 5} \tilde{y}\right)$ now, with $\tilde{y}$ of $O(1)$. This last scale is due to the shrinking $\propto\left(T_{0}-T\right)^{3 / 4}$ of the previous normal scale of order $\epsilon^{5}$. 
In $(7.2 a-c) \tilde{\gamma}, p_{0}, d_{0}, d_{1}$ and the integral factor $I_{1}$ are $O(1)$ constants again. The $\bar{y}$ momentum balance shows that $P_{2}, P_{3}, P_{4}$ are independent of $\tilde{y}$, as expected. The matching conditions include

$$
\begin{gathered}
\tilde{u}_{0} \sim-6 b_{3} b_{1}^{-2} d_{1} \tilde{t} \tilde{y}+O(\ln |\tilde{y}|), \\
\tilde{u} \sim b_{3} \tilde{y}^{3}+6 b_{3} b_{1}^{-2} P_{2} \tilde{y}+O(\ln |\tilde{y}|), \\
\tilde{v} \sim-3 b_{3} b_{1}^{-2} P_{2 \tilde{x}} \tilde{y}^{2}+O(\tilde{y} \ln |\tilde{y}|)
\end{gathered}
$$

as $|\tilde{y}| \rightarrow \infty$. The solution outside the critical layer remains to some extent a Taylor expansion of that in step 2 as $(X, T) \rightarrow\left(X_{0}, T_{0}\right)$, as described below. Substitution of $(7.1 a)-(7.2 c)$ into the Navier-Stokes equations then leads to the continuity and $\bar{x}$-momentum balances

$$
\begin{gathered}
\tilde{u}_{0 \tilde{x}}+\tilde{v}_{0 \tilde{y}}=0, \\
\tilde{u}_{0 \tilde{t}}+--++b_{1} \tilde{y} \tilde{u}_{0 \tilde{x}}+b_{1} \tilde{v}_{0}-b_{1}^{-1} P_{2 \tilde{x}} \tilde{u}_{0 \tilde{y}}=0 .
\end{gathered}
$$

Differentiation of $(7.4 b)$, and the normalization

$$
\left[P_{2}, \tilde{x}, \tilde{y}, \tilde{t}\right]=\left[-\hat{d} \ell^{3} \hat{P},-\ell \hat{x},-\frac{\ell^{3 / 2} \hat{d}^{1 / 2}}{b_{1}} \hat{y}, \frac{\hat{t}}{\ell^{1 / 2} \hat{d}^{1 / 2}}\right],
$$

where $\hat{d}=d_{1} a_{1} /\left(6 a_{3}\right)$ is assumed positive for now and $\ell$ is a length factor, give the governing equation

$$
\hat{\tau}_{\hat{t}}+\hat{y} \hat{\tau}_{\hat{x}}+\hat{P}_{\hat{x}} \hat{\tau}_{\hat{y}}=0,
$$

with the constraints, inter alia,

$$
\hat{\tau} \sim \hat{t}+O\left(\hat{y}^{-1}\right) \text { as } \hat{y} \rightarrow \pm \infty,
$$

for the local normalized vorticity $\hat{\tau}$. The critical-layer dynamics in $(7.5 a, b)$ is coupled in general with the flow solution outside, in the bulk of the motion where the normal scale is larger, as studied in the next paragraph. There an analogue of the working for step 2 shows that the normalized pressure $\hat{P}(\hat{x}, \hat{t})$ and the normalized velocity jump across the critical layer,

$$
\hat{J}(\hat{x}, \hat{t}) \equiv f_{-\infty}^{\infty} \hat{\tau} \mathrm{d} \hat{y},
$$

both of which are unknown, are linked now by the relation

$$
-6=\hat{P}_{\hat{x} \hat{x} \hat{x}}-6 \tilde{\mu} \hat{J}_{\hat{x}},
$$

cf. (3.7), (4.7), subject to matching at large $|\hat{x}|$ which is similar to that in step 2. Thus the inner-outer interaction is described generally by $(7.5 a-d)$ for $\hat{\tau}(\hat{x}, \hat{y}, \hat{t}), \hat{J}(\hat{x}, \hat{t}), \hat{P}(\hat{x}, \hat{t})$. Comparison with step 2 shows that in fact there is an overtake of the critical-layer terms between steps 2,3 in a time scale of order $\phi^{81 / 7}$, i.e. when $\hat{t}$ is large, negative and of order $\phi^{-8 / 35}$, although this overtake is a relatively passive affair concerning only higher-order terms prior to step 3 .

The flow in the bulk outside has $\bar{y}=\epsilon^{5} y$, and the expansion (from step 2)

$$
\begin{gathered}
\bar{u}=\epsilon u_{0}(y)+\phi^{11 / 2} \tilde{U}_{11}(y)+\phi^{63 / 10} \tilde{U}_{12}+\phi^{67 / 10} \tilde{U}_{1}+\phi^{15 / 2} \tilde{U}_{2}+\phi^{79 / 10} \tilde{U}_{3}+\ldots, \\
\bar{v}=\phi^{73 / 10} \tilde{V}_{1}+\phi^{81 / 10} \tilde{V}_{2}+\phi^{17 / 2} \tilde{V}_{3}+\ldots \\
\bar{p}=\epsilon^{2} p_{0}+\phi^{9} d_{0}-\phi^{98 / 10} d_{1} \tilde{t}+\phi^{102 / 10} \tilde{P}_{2}+\phi^{11} \tilde{P}_{3}+\phi^{114 / 10} \tilde{P}_{4}+\ldots
\end{gathered}
$$


The term associated with the main jump $\hat{J}$ above is $\tilde{U}_{3}$ in (7.6a). Again from the Navier-Stokes equations the streamwise momentum equations for $\left(\tilde{U}_{1}, \tilde{V}_{1}, \tilde{P}_{2}\right)$, $\left(\tilde{U}_{2}, \tilde{V}_{2}, \widetilde{P}_{3}\right)$ are as in step 2 , while the normal momentum balances require $\widetilde{P}_{2}, \widetilde{P}_{3}$ to be independent of $y$ and hence equal to $P_{2}, P_{3}$ in (7.2c). The jump term is then governed by

$$
\begin{aligned}
\tilde{U}_{3 \tilde{x}}+\tilde{V}_{3 y_{b}} & =0, \\
\left(u_{0}-c\right) \tilde{U}_{3 \tilde{x}}+\tilde{V}_{3} u_{0 y_{b}} & =-\tilde{P}_{4 \tilde{x}}-\tilde{U}_{12 \tilde{t}},
\end{aligned}
$$

where $\tilde{U}_{12}$ is independent of $\tilde{x}$, and the normal-pressure-gradient effect

$$
\left(u_{0}-c\right) \tilde{V}_{1 \tilde{x}}=-\tilde{P}_{4 y_{b}} \text {. }
$$

Integration of $(7.7 a-c)$ then confirms the result $(7.5 d)$, given that $\tilde{U}_{12}=d_{1} \tilde{t} u_{0}$ and the definitions of the integrals $a_{1}, a_{3}$ (which is positive) in $\$ 3$.

The boundary conditions in $\hat{x}$, on $(7.5 a-d)$, are of the form $\hat{P}=-O\left(\hat{x}^{3}\right)$ at large $|\hat{x}|$, similar to those in $\S 4$ where the influence of the factor $\ell$ and the functions of integration implied by $(7.5 d)$ are first discussed. The argument here runs similarly to that in $\S 4$. There are at first sight three arbitrary functions of $\hat{t}$ admissible through integration with respect to $\hat{x}$ in $(7.5 d)$, e.g. from considering an iterative solution. Of these, one can be absorbed into $\hat{P}$ as an origin shift without altering $(7.5 a-d)$. A second can be absorbed as an origin shift in $\hat{x}$ but accompanied by other redefinitions. Specifically the subtraction of a function $h^{\prime \prime}(\hat{t})$ say from $\hat{P}_{\hat{x}}$ is cancelled out by subtracting $h(\hat{t})$ from $\hat{x}$ and $h^{\prime}(\hat{t})$ from $\hat{y}$, to leave $(7.5 a-d)$ intact again. These shifts can be generalized further if necessary. The third and final function of $\hat{t}$ is associated to some extent with the length factor $\ell$ which does not appear in (7.5a-d). Here interest can be confined for example to a solution connected with $\hat{P}=-\hat{x}^{3}+\hat{x} \hat{t}$, which produces a vortex wind-up starting at zero time $\hat{t}$, for zero $\tilde{\mu}$. This requires, incorporating the shifts above,

$$
\hat{P}_{\hat{x}}=-3 \hat{x}^{2}+\hat{t}+6 \tilde{\mu} f_{-\infty}^{\hat{x}} \hat{J} \mathrm{~d} \hat{x}
$$

as the twice integrated form of $(7.5 d)$. In (7.8) however the term $\hat{t}$ in general can be replaced by a general function of $\hat{t}$, and indeed extensions of (7.8) indicate a solution dependence on the local streamwise boundary conditions as well as on the value of $\tilde{\mu}$. Also in (7.8) the double bar on the integral sign denotes that terms of order unity as $\hat{x} \rightarrow-\infty$ are to be omitted; we note that $\hat{J}$ is in fact $O\left(\hat{x}^{2}\right)$ at large $|\hat{x}|$, from the match with $\S 4$. In similar fashion the integrand in $(7.5 c)$ could be replaced by $(\hat{\tau}-\hat{t})$ but with the bar still present because of the $O\left(\hat{y}^{-1}\right)$ decay of $(\hat{\tau}-\hat{t})$.

The solution of the fundamental problem $(7.5 a-d)$ matches with that in $\S 4$ as $\hat{t} \rightarrow-\infty$, as required, with the first term in (7.5a) then exerting a secondary influence apart from an additive constant, giving precisely the previous jump $\hat{J}$ (for $T \rightarrow T_{0}-$ ) in step 2. For $O(1)$ times $\hat{t}$ it is less simple. Some insight comes from the characteristics or trajectories of $(7.5 a)$, given by $\mathrm{d} \hat{x} / \mathrm{d} \hat{t}=\hat{y}$ and $\mathrm{d} \hat{y} / \mathrm{d} \hat{t}=\hat{P}_{\hat{x}}$, i.e. $\mathrm{d} \tilde{y} / \mathrm{d} \tilde{t}=-b_{1}^{-1} P_{2 \tilde{x}}$, so that $\mathrm{d}^{2} \hat{x} / \mathrm{d} \hat{t}^{2}=\hat{P}_{\hat{x}}$, on each solution curve of which $\hat{\tau}$ is constant from $(7.5 a)$. The trajectories' equation cannot be solved explicitly, however. Numerical solutions of $(7.5 a-d)$ were sought directly but proved difficult to obtain accurately especially in view of the complex far-field behaviour of $\hat{\tau}$ at large $|\hat{x}|$ and/or $|\hat{y}|$, and the results were unclear. A more analytical approach, for small $\tilde{\mu}$, was found to be more enlightening, and this is summarized below (further details are available from the authors). 
For small $\tilde{\mu},(7.5 d)$ gives the simple pressure result $\hat{P}=-\hat{x}^{3}+\hat{x} \hat{t}$ at leading order, on the time scale $\hat{t}$ of $O(1)$. That leaves $(7.5 a, b)$ to be solved for $\hat{\tau}(\hat{x}, \hat{y}, \hat{t})$, given $\hat{P}$ just above, which sets a generic problem. Solutions for $\hat{\tau}$, however, found from the behaviour of the particle trajectories (figure 7a), give the jump $\hat{J}$ from $(7.5 c)$ growing in the form $\hat{J} \propto \hat{t}^{7 / 4}$ at large positive $\hat{t}$, while typically $|\hat{x}| \propto \hat{t}^{1 / 2}$; see figure $7(b)$. The growth means that eventually the $6 \tilde{\mu} \hat{J}_{\hat{x}}$ term in $(7.5 d)$ becomes comparable with the others. That happens when $\hat{t}$ increases to $O\left(|\tilde{\mu}|^{-4 / 5}\right)$, by which stage the vortex is strongly wound up (figure $7 a$ ). The implied next stage for small $\tilde{\mu}$ has the longer time scale $\hat{t}=|\tilde{\mu}|^{-4 / 5} \bar{t}_{0}$ with $\bar{t}_{0}$ of order unity for the unknown captured trajectories, and inner-outer interaction re-appears. The typical subsequent response then depends mostly on the sign of $\tilde{\mu}$ and shows that the vortex wind-up either strengthens even more, to yield an explosive growth (figure $7 c$ ) in its area and length, or it ultimately weakens, to produce an implosion (figure $7 d$ ). The former leads to a singular termination at a finite value of $\bar{t}_{0}$ and the latter to a decaying form as $\bar{t}_{0} \rightarrow \infty$.

The implied terminal behaviour for order-one values of $\tilde{\mu}$ is as follows. First, a finite-time singularity can occur in which, for the majority of the strong (expanding) vortex, the scaled vorticity, pressure, velocity jump and length scales satisfy

$$
[|\hat{\tau}-\hat{t}|, \hat{P}, \hat{J}, \hat{x}, \hat{y}] \sim\left[\left(\hat{t}_{e}-\hat{t}\right),\left(\hat{t}_{e}-\hat{t}\right)^{-6},\left(\hat{t}_{e}-\hat{t}\right)^{-2},\left(\hat{t}_{e}-\hat{t}\right)^{-2},\left(\hat{t}_{e}-\hat{t}\right)^{-3}\right]
$$

as time $\hat{t}$ approaches the finite time $\hat{t}_{e}$. This is usually for negative $\tilde{\mu}$ values although there is dependence also on the precise streamwise boundary conditions, referring back to (7.8). Second, and usually for positive $\tilde{\mu}$, a decaying vortex is produced as $\hat{t} \rightarrow \infty$, its scaled length $\overline{\bar{L}}$ and area $\overline{\bar{a}}$ being such that

$$
[\overline{\bar{L}}, \overline{\bar{a}}] \sim\left[\hat{t}^{-5 / 4}, \hat{t}^{-5 / 2}\right]
$$

then. The terminations $(7.9 a, b)$ are in line with the computed results in figure $7(c, d)$ respectively.

\section{Further comments}

This work has studied the impact of normal pressure gradients (step 2) and then vortex winding-up (step 3) typically which come into force, with shortened length and time scales, beyond the finite-time break-up of the interacting boundary-layer stage (step 1). Further comments are as follows.

\subsection{Comments on step 2}

The main governing equation of step 2 is the supplemented $\mathrm{KdV}$ or Benjamin-Ono equation, balancing the momentum in effect integrated normally across the entire local flow including the critical layer against the integrated normal pressure difference and producing a controlling parameter $\mu$ (see (iii) below), along with the choice of $\Delta$ or $a_{3}$. The governing equation holds irrespective of the local pressure-displacement interaction law. For the special internal-flow case of negligible $\Delta$ on which the work of $\S \S 4-6$ concentrates (cf. the external case (3.14) which is described later in this subsection), three distinct types of solution path are found as time increases depending on whether $\mu$ is positive, negative or zero.

For positive $\mu$, associated with $\operatorname{sgn}\left(\tilde{\mu} a_{4}\right)$ being positive (in practice we took the normalized constants $\tilde{\mu}, a_{4}$ as both positive then), $\S 4$ indicates that the flow solution 
(a)

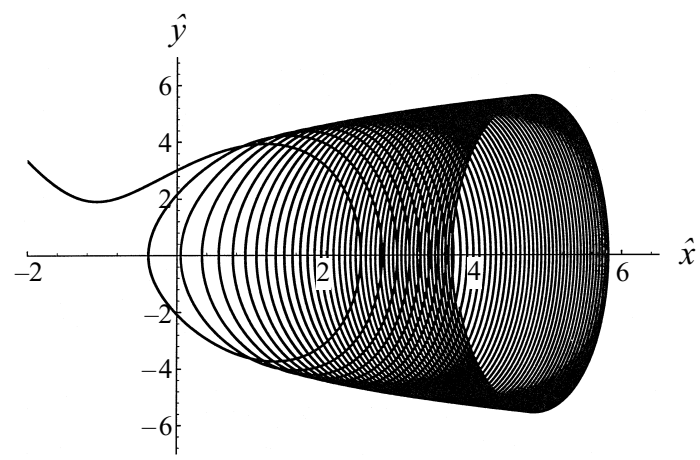

(b)

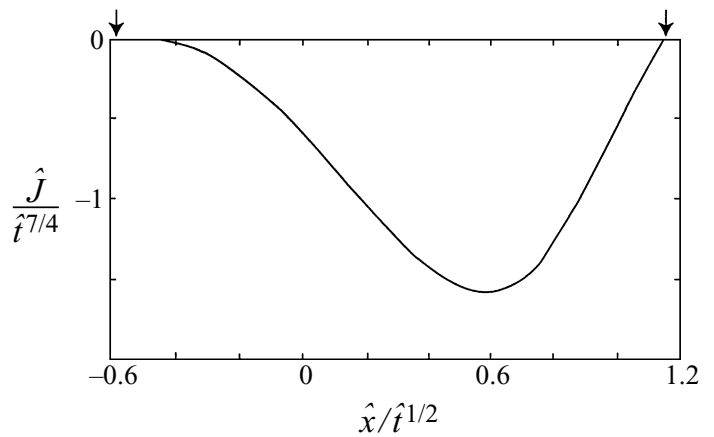

(c)

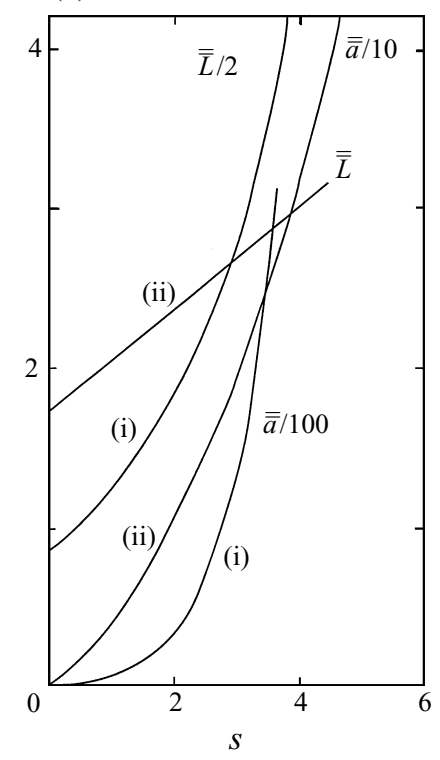

(d)

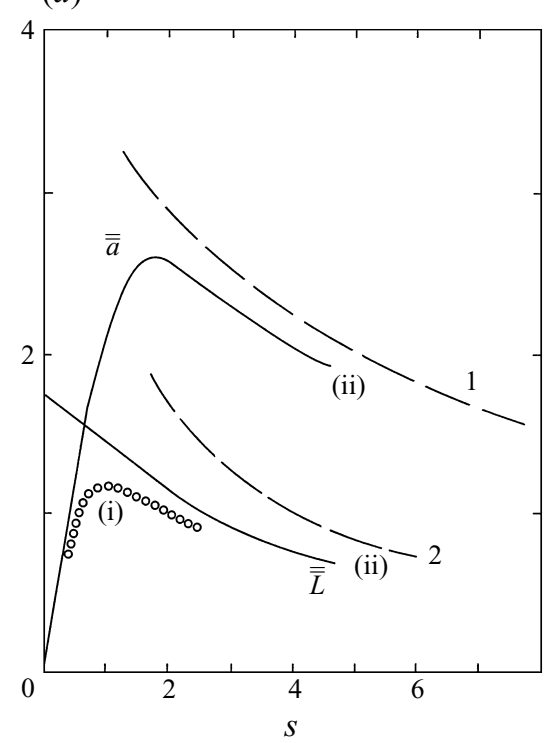

FIGURE 7. For caption see facing page. 
becomes singular within a finite value of the new scaled time. This termination happens at the onset of a maximum/minimum in the scaled surface pressure, a matter followed up in $\S 7$. The precise terminal form obtained in $\$ 4$ holds only for a finite range of $\tilde{\mu}$ values but Appendix B provides a guide to the terminal form outside that range and in any case much interest is in the sub-range of small $\tilde{\mu}$ values (e.g. see $\S 7)$, which is covered by $\S 4$. Another interesting detail is the increasing steepness of the pressure curves, around $X=0$ in figure $2(a, b)$, as $\tilde{\mu}$ is increased, suggesting perhaps a subtle interplay eventually with the above onset.

It is interesting that the terminal form in $\$ 4$ also applies in principle to a finite range of negative $\mu$ values (i.e. $\tilde{\mu}, a_{4}$ of opposite signs), for $\S 5$ shows the timemarching problem for negative $\mu$ to have strong secondary instability present which raises inherent difficulties with regard to well-posedness. It could be argued that with sufficiently smooth input data the terminal singularity as in $\$ 4$ is still relevant then of course, but in general much shorter-scale physics must come into the reckoning, and at earlier times. (This, we repeat, is for the extended $\mathrm{KdV}$ case.) For zero $\mu$ on the other hand $(\S 6)$ the standard $\mathrm{KdV}$ equation applies. The boundary conditions appear non-standard, however, and they induce a mixture of solitary and non-solitary waves, and of two coupled streamwise length scales, at large positive scaled times. The length scales, it can be shown, are such that the next step encountered reinstates the interacting boundary-layer system on the longer scale (as in \$2) but, on the shorter scale, provokes the Euler system, and these two systems are coupled together: when time $T$ becomes as large as $R e^{4 / 7}$ in this internal-flow context the longer scale in $\S 6$ increases by a further $R e^{6 / 7}$ whereas the shorter scale decreases by a factor $R e^{-1 / 7}$. Incorporating the $\bar{x}$ scaling of $\S 3$ we therefore obtain the two streamwise length scales $O(R e)$ (as in $\S 2$ ) and $O(1)(=O(\bar{y}))$, respectively, and hence the two systems above. The coupled system above likewise merits further study. A similar coupling occurs in the theory on spots in Smith et al. (1994), who note the likelihood of further sublayer bursting(s) arising between the shorter-scale Euler flows and the surface.

The boundary conditions on the $\mathrm{KdV}$ case in $\S 6$, incidentally, may appear nonstandard but in fact there are clear applications to water waves and to the formation of bores. On long distance and time scales, proportional to the Reynolds number (as in $(2.3 d)$ ), a viscous liquid layer is controlled by the interactive boundary-layer equations across the depth of the fluid with unknown pressure proportional to the unknown depth, cf. the shallow-water balance. These have been studied by Bowles $(1990,1995)$, Higuera (1994) and actually correspond to step 1 of the present Introduction. If the unsteady form of the equations is considered then the break-up of $(2.4 a-d)$ leads to the Burns (1953) formula

$$
\int_{0}^{h} \frac{1}{\left(u_{0}-c\right)^{2}} \mathrm{~d} y=F r
$$

FIGURE 7. On main properties in step 3 , for small $|\tilde{\mu}|$. (a) Plot of $\hat{y}$ vs. $\hat{x}$ for a particle trajectory captured in the vortex, over the range $2 \leqslant \hat{t} \leqslant 70$. (b) Jump $\hat{J} / \hat{t}^{7 / 4}$ against $\hat{x} / \hat{t}^{1 / 2}$, computed from $\hat{\tau}$ profiles, at large $\hat{t}$. Arrows show the vortex ends. (c) Computed results, versus $s\left(\equiv|\tilde{\mu}| \hat{t}^{5 / 4}\right)$, for the scaled area $\overline{\bar{a}}$ and length scale $\overline{\bar{L}}$ of the evolving vortex in two representative cases (i), (ii) when $\tilde{\mu}$ is negative. The area and length in terms of $\hat{x}, \hat{y}$ coordinates are $|\tilde{\mu}|^{-1} \overline{\bar{a}},|\tilde{\mu}|^{-2 / 5} \overline{\bar{L}}$ respectively. (d) The scaled vortex area $\overline{\bar{a}}$ and length $\overline{\bar{L}}$ computed for positive $\tilde{\mu}$, and corresponding asymptotes 1,2 for large $\tilde{\mu}^{4 / 5} \hat{t}$. Results shown are all for case (ii) except that the computed $\overline{\bar{a}}$ variation for (i) is also indicated, by circles, for comparison. 
for the break-up criterion. Here $h$ is the local depth and $F r$ is the Froude number, assumed to be of order one. This criterion can be met for all values of $F r$ with $c<0$ and $c>u_{\max }$, the maximum value of $u_{0}$, corresponding to up- and downstream travelling bores respectively. There is also the possibility of roots with $0<c<u_{\max }$ depending on the form of $u_{0}$. There is no critical layer in the former cases, so that $\mu=0$ and the next stage, stage 2, leads to the familiar KdV scales for long waves. The pressure gradients (and hence wave slopes) become $O\left(R e^{-3 / 7}\right)$ rather than $O\left(R e^{-1}\right)$ and locally the skin friction is increased to $O\left(R e^{1 / 7}\right)$. The bore, consisting of a leading set of solitary waves and followed by a train of cnoidal waves, then develops according to the description of $\$ 6$ and Appendix C. At large times the wave train lengthens to be $O(R e)$ in length, but the individual waves themselves become short and steep so that the $\mathrm{KdV}$ equation is no longer adequate to describe them; this process is analogous with the long-short coupling discussed in the previous paragraph.

Some research is also in progress on the three-dimensional version of the current work, starting with the three-dimensional extension of the finite-time singularity of $\S 2$ in Hoyle \& Smith (1994). Several aspects of this need more consideration including the likelihood that there are a number of possible break-up terminations of step 1 for a three-dimensional unsteady interacting boundary layer. The three-dimensional extension into steps 2,3 should also be tackled.

Likewise, other cases of internal/external motion exist (e.g. entry flows), including mixtures of the internal and external contexts of $(3.12 a)$, but the present externalflow case (3.14) with its extended Benjamin-Ono system appears to have the widest application. Computed solutions for (3.14) (with (3.12a)) are presented in figure 8 for various $\tilde{\mu}, a_{5}$ values, in the normalized form for which $\left(a_{1}, a_{2}, a_{3}, \mu, \theta_{2}\right)$ in $(3.12 a)$ take the values $\left(1,1,0, \tilde{\mu}, a_{5}\right)$ in effect, in view of a transformation analogous with that in (4.1). More details on the compact differencing used here are given by Li (1997). The numerical solution for zero $\mu$ exhibits a packet of travelling waves and can be maintained for a very long time with sufficiently small grid sizes and time steps (similarly to $\S 6)$; see figure $8(a-d)$. We observe that here the eventual direction of travel clearly reverses. Large-time features are again given in Appendix C. For non-zero $\mu$, opposite signs of $\tilde{\mu}, a_{5}$ yield stable behaviour leading on to a finite-time singularity, similar to that in $\S 4$, whereas with $\tilde{\mu}, a_{5}$ having the same sign the solution exhibits a secondary instability phenomenon akin to that of $\S 5$ (in particular $-\tilde{\mu} a_{4} \alpha^{3}$ in (5.3) is replaced now by $\left.+\tilde{\mu} a_{5} \alpha|\alpha|\right)$. Here we present just four computational examples to demonstrate the point; see figure $8(e-h)$, where $L, M$ denote the approximations for the two Cauchy integrals in $\tilde{\mu}, a_{5}$ respectively. Thus the sensitive dependence (in the special case in $\S \S 4-6)$ on the parameter $\mu$ is repeated here in the more general case but with the roles of $\mu$ positive or negative being reversed. Since the most physically sensible profiles give the curvature parameter $\mu\left(\equiv 6 b_{3} b_{1}^{-4}\right)$ being negative as in figure 1 for example, it follows also that in this more common, extended Benjamin-Ono, case secondary instability is not encountered and instead a termination as in $\S 4$ is relevant. That in turn also points to the subsequent study of wind-up in $\S 7$ as being typical.

\subsection{Comments on step 3}

Step 3 involves the new unsteady, interactive, critical layer effects that come into action in a fundamental problem of local vortex wind-up (\$7); this, we repeat, is for the special internal flow but it yields many generic features. Although the secondary instability in $\$ 5$ strictly limits the relevance of $\$ 7$ to positive $\mu$ for that special flow, the opposite is true for the more general external or quasi-external flow, as indicated at the end of $\S 8.1$ above, for which negative $\mu$ values are more sensible physically 
(a) $\tilde{p}(X, T)$

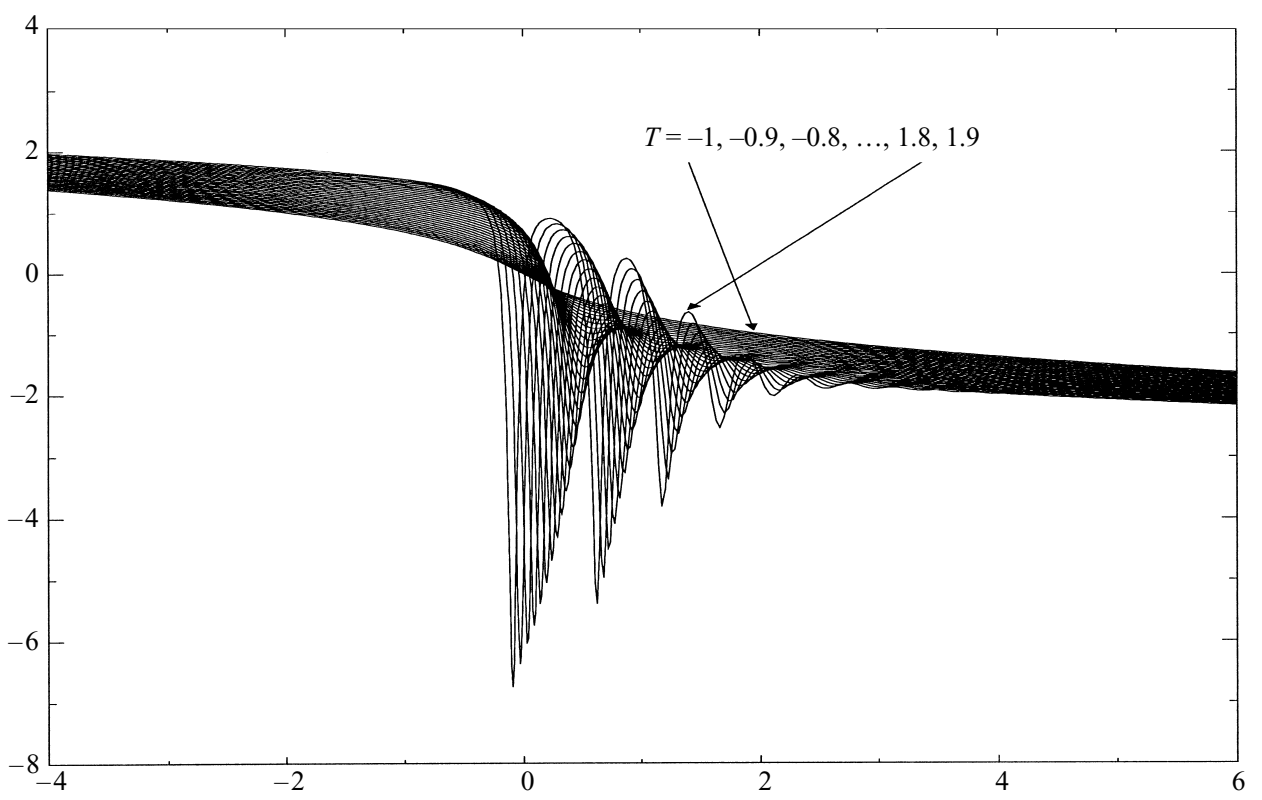

(b) $M(X, T)$
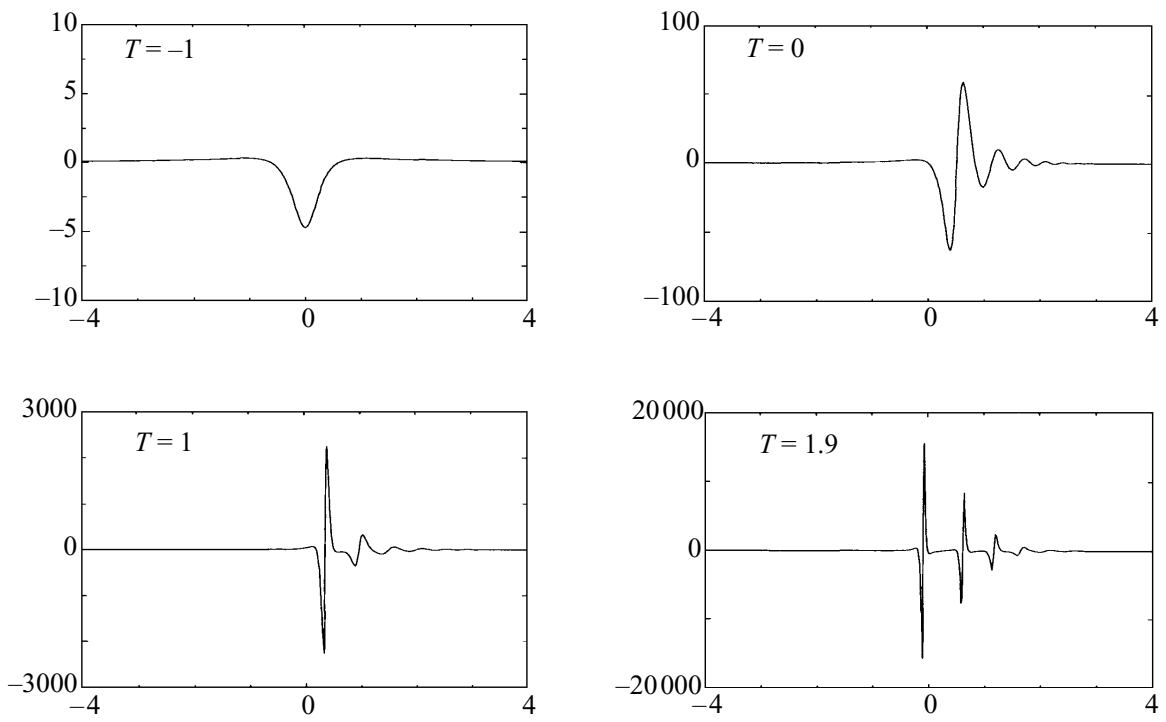

FIGURE $8(a, b)$. For caption see page 367.

anyway. Thus again step 3 represents the typical next step. No further secondary instability is found, step 3 indicating instead two different vortex evolutions arising at large $R e$ and depending largely on the sign of $\mu$. There is also some dependence on the streamwise boundary conditions (in $\$ \S 4,7$ ), implying that the flow further upstream and downstream still manages to exert some local influence. Each type of evolution however is unaffected ultimately by earlier, historical, vorticity values, to leading order, unlike in the Brown \& Stewartson (1978) setting. 
(c) $\tilde{p}(X, T)$

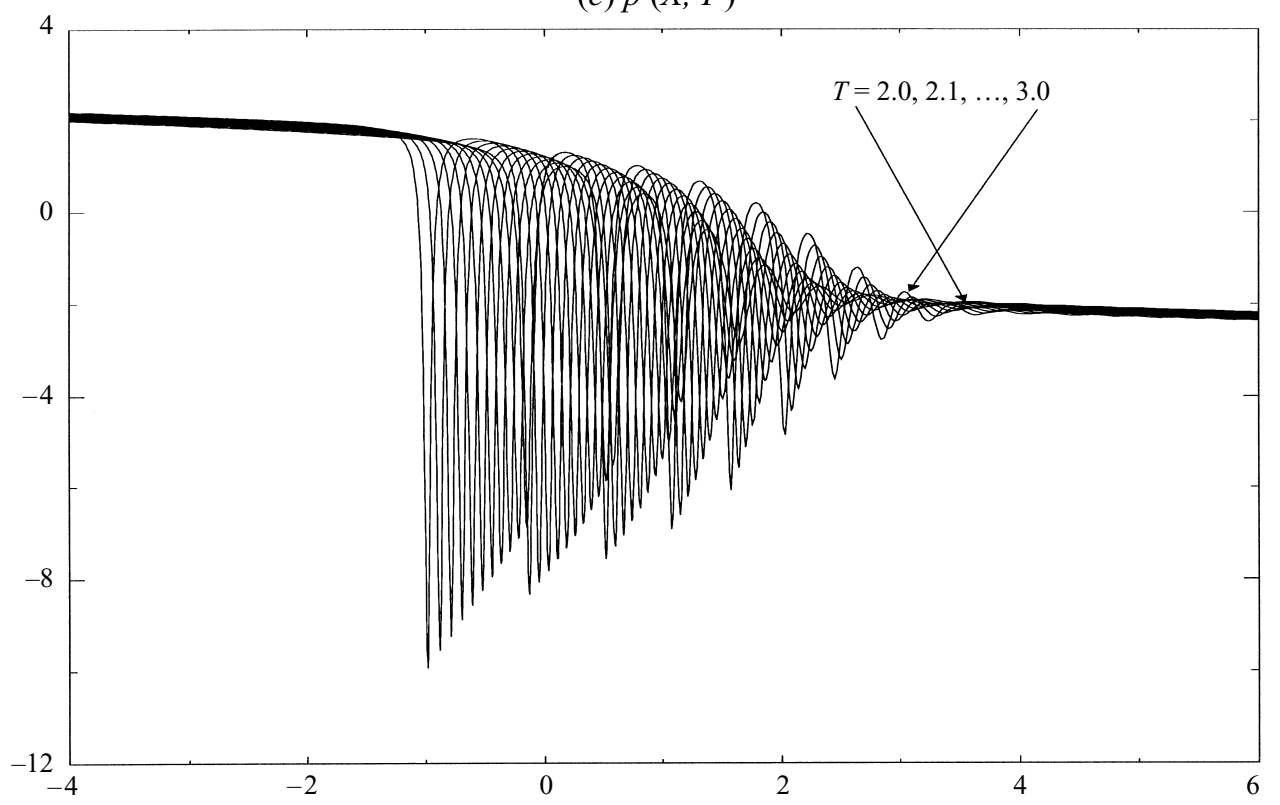

(d) $\tilde{p}(X, T)$

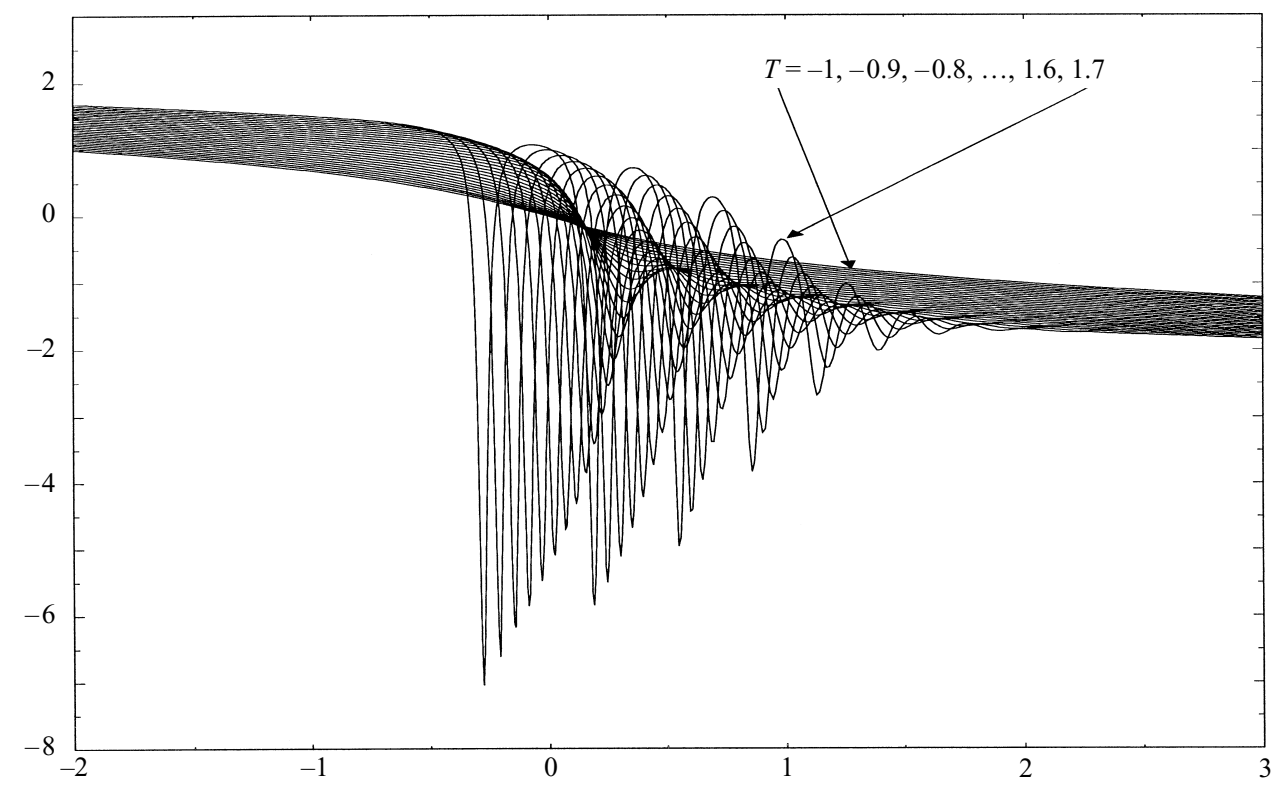

FigURE $8(c, d)$. For caption see page 367.

The next stage, step 4 , may be expected to have the fuller response $\partial \hat{P} / \partial \hat{t}+\hat{P} \partial \hat{P} / \partial \hat{x}$ essentially replacing the constant contribution on the left in $(7.5 d)$, a contribution which stays in play at the termination of step 3 in $\S 7$. The vortex evolutions for small $\mu$ in step 3 could also shed light on multiple vortices, when connected with the simpler 
(e)
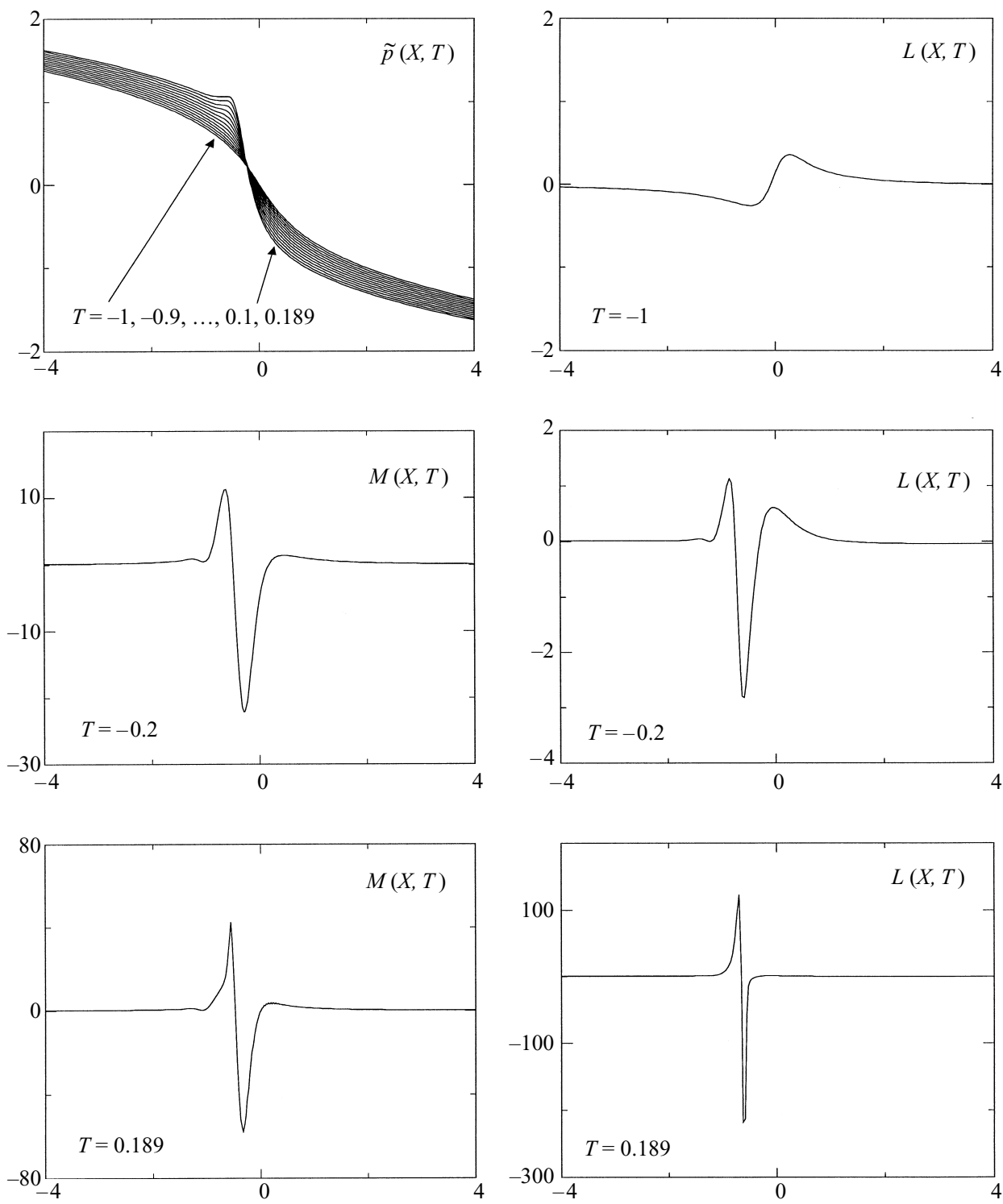

FigURE 8(e). For caption see page 367.

$\mathrm{KdV}$ and Benjamin-Ono regimes of $\S 6$ and $\S 8.1$ above; in the more general extended Benjamin-Ono context the triple-derivative term in $(7.5 d)$ is replaced by a Cauchy integral, a case meriting future study.

\subsection{On the scales and parameters}

The controlling scales are indeed rather bizarre, and in practice some extreme ones may only emerge at very large $R e$ values, but the appearance of $1 / 7$ and $1 / 5$ powers of $R e$ in step 3, for the internal case, is not surprising, in view of the combined importance then of the normal pressure gradients and the critical-layer dynamics. The study of the complicated physical mechanisms entering, via the distinct temporal 
$(f)$
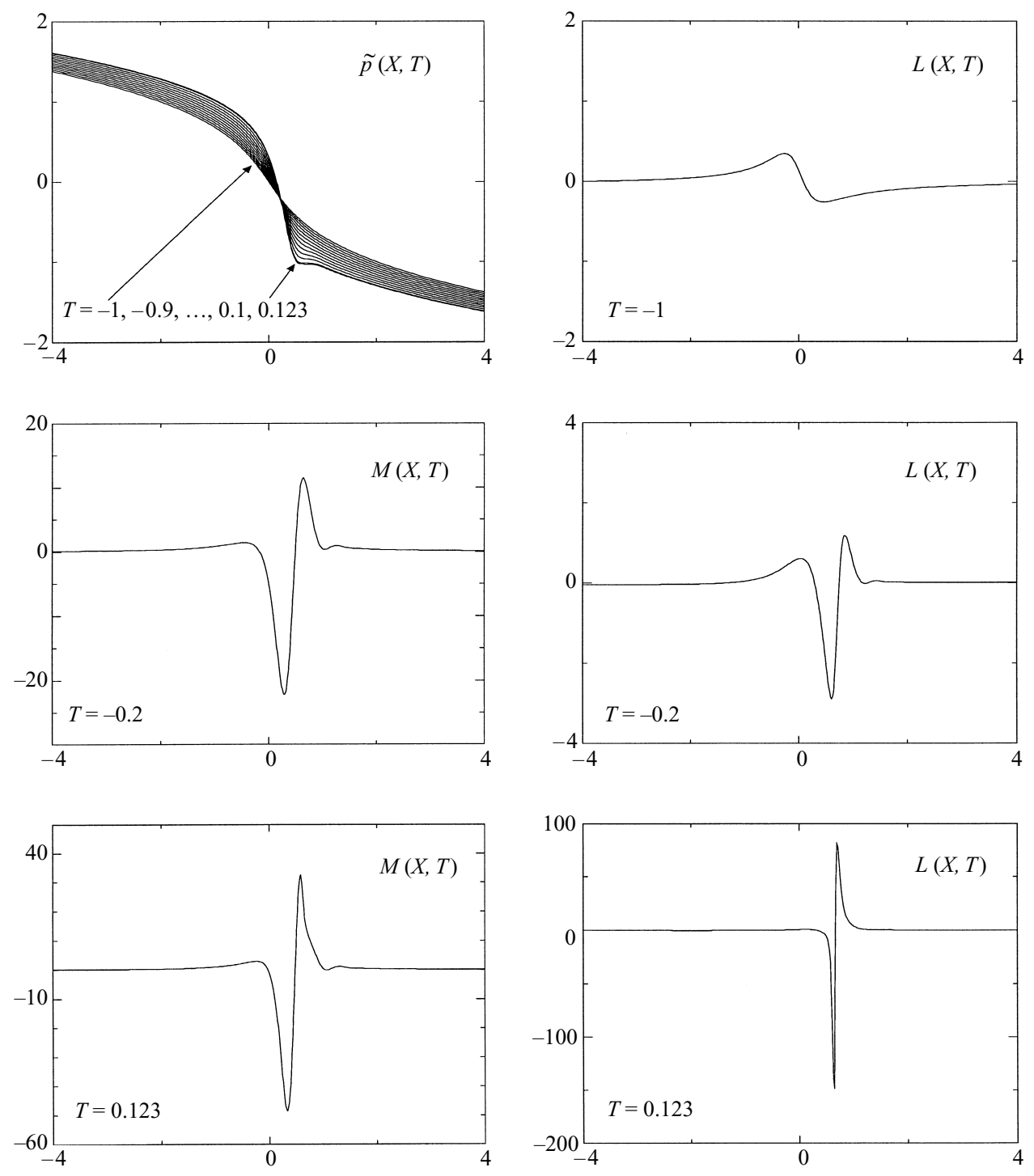

Figure $8(f)$. For caption see facing page.

steps $1,2,3$, etc., provides additional intuitive insight into the local flow dynamics including the vortex wind-up. In particular the sign of the parameter $\mu$ is identified as key throughout. This is because of the clear dependence on $\operatorname{sgn}\left(\tilde{\mu} a_{4}\right)$ in $\S \S 4-7$, combined with the relation $\tilde{\mu} a_{4}=\left(\mu a_{3}\right) /\left(a_{1}^{2} \Gamma^{7 / 2}\right)$, in which both the denominator and the constant $a_{3}$ are positive, the latter from manipulation of the integral definition of $a_{3}$ in $\S 3$. The value of the key parameter $\mu$, to reiterate, comes directly from the local non-dimensional velocity profile $u_{0}(y)$, as $\mu=6 b_{3} / b_{1}^{4}=u_{0}^{\prime \prime \prime} /\left(u_{0}^{\prime}\right)^{4}$ at the critical level $y=y_{0}$. In consequence, the distinctions between the flow properties for positive and negative $\mu$, in steps 2,3 , whether for the special internal context or for the contrasting more general context (see end of §8.1), depend solely on the curvature-gradient term $b_{3}$, which is negative in most physical configurations. 
$(g)$
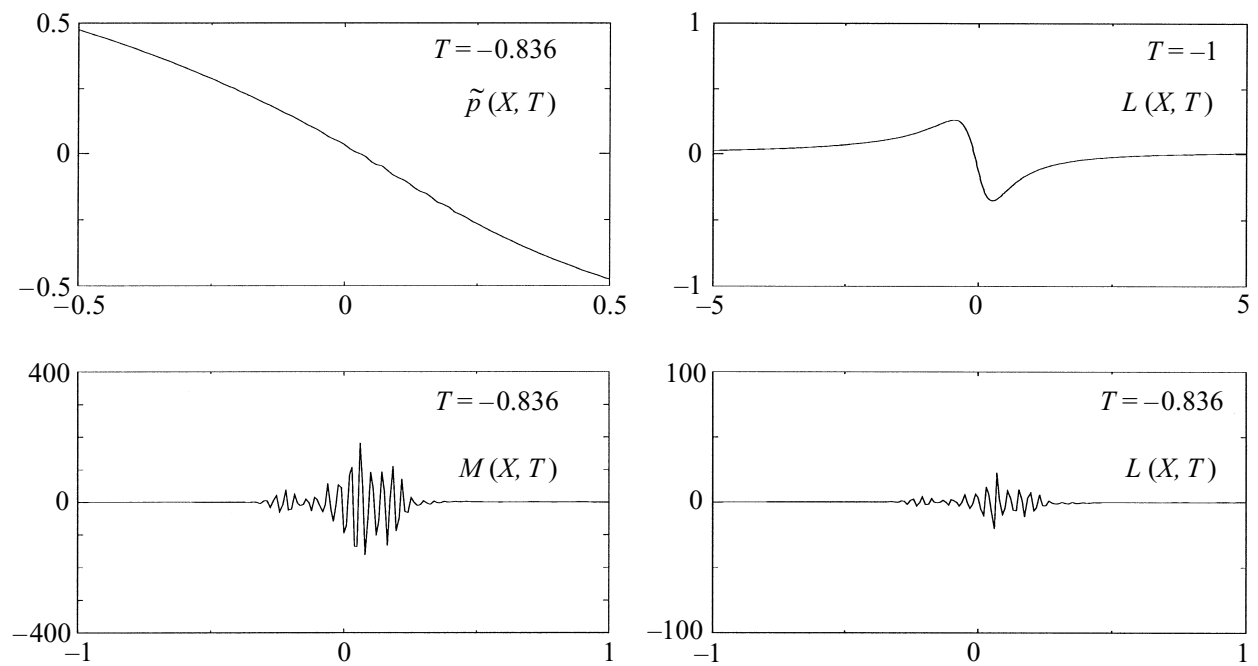

(h)
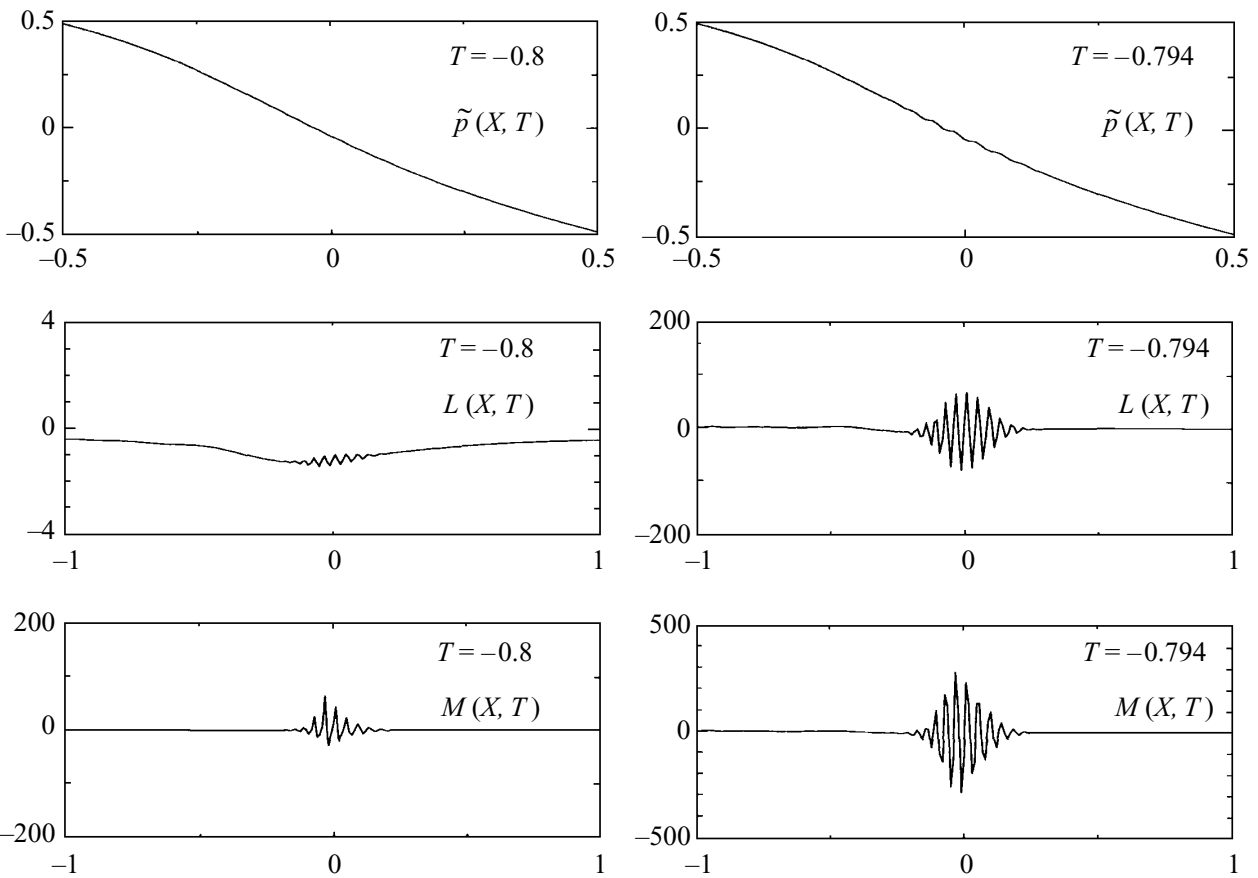

FIGURE 8. Numerical solutions for the extended Benjamin-Ono case, i.e. (3.12a) with $\left(a_{1}, a_{2}, a_{3}, \mu, \theta_{2}\right)=\left(1,1,0, \tilde{\mu}, a_{5}\right)$; see $\S 8:(a-c) \tilde{\mu}=0, a_{5}=-0.1$, showing $(a, c) \tilde{p},(b) M$. (d) has $\tilde{\mu}=0, a_{5}=-0.05$, showing $\tilde{p} ;(e-h)\left(\tilde{\mu}, a_{5}\right)$ equal to $(-0.1,0.1),(0.1,-0.1),(-0.1,-0.1),(0.1,0.1)$ in turn, showing $\tilde{p}, L, M$.

\subsection{Links between steps 1-3 and experiments or simulations}

The repercussions for direct or indirect numerical simulations seem to be fairly straightforward: the simulations should be arranged so as to acknowledge the (sometimes delicate) scales and interactions shown by the theory, if accuracy is to be 
preserved at large $R e$. The current work possibly supports more the use of reduced equation sets, rather than the usual direct simulation approaches, for large Re, perhaps along with zonal methods, for example. In either case the theory indicates in principle the grid-resolution requirements needed to capture accurately the small-scale properties of the local dynamics that control for instance vortex formation within the boundary layer. The composite system consisting of the interacting boundary-layer equations but coupled with $\partial v / \partial t+u \partial v / \partial x=-\partial p / \partial y(p=p(x, y, t)$ now $)$, for instance, would appear to accommodate all the dominant physical features found theoretically in steps 2, 3 and possibly 4. Some studies of that composite system are in Smith, Papageorgiou \& Elliott (1984), Smith (1991).

Meanwhile qualitative and quantitative links of the theory with numerical simulations as well as with experiments have already been described near (1.1), (1.2), for step 1 (and partly step 2). Another link, concerned with steps $1-3$, is as follows. Using the technique of wavelet transforms Gaster (1993) and Shaikh \& Gaster (1994) identify four disturbance frequencies becoming active as transition deepens in modulated TS wavepackets. A tentative comparison can be made between the above experimental measurements and the theory pursued in this paper. In addition to the basic TS frequency there is some evidence in the nonlinear development prior to spiking of a subharmonic component. This we would identify with the vortex of the three-dimensional nonlinear wave-vortex interaction described by our step 1 of the Introduction. As transition deepens, localized bursts of oscillations at 5-6 times the TS frequency are observed and identified as the first spikes. Their appearance is deterministic in nature, although their fine structure does not seem to be. This third frequency may be associated with the current step 2. The highest frequency, at 20 times the fundamental, is seen to arise within these spikes as transition deepens further. One possible interpretation is that this is the frequency associated with the vortex roll-up described in step 3 (\$7). However, the evolution of this frequency component is found experimentally not to be deterministic and an alternative transition path such as in the initial-value setting of Savin (1996), Smith (1996), Savin et al. (1998) may be being followed at this stage and possibly in step 2 also.

Further, Sandham \& Kleiser's (1992) channel-flow computations, showing a number of vortices being produced as transition deepens, raise the matter of a theoretical explanation of the continued strengthening of certain vortices compared with others. Brinkman \& Walker (1996) also report multiple vortices appearing, in company with rapid wall-layer breakdown, in their more recent model simulations. Again, an explanation seems desirable for the effectiveness of using the pressure and in particular its maxima and minima to monitor the creation of vortices in later transition (see also Wintergerste \& Kleiser 1995 and near (1.1)), and likewise for the impact of vortex interactions, in Sandham \& Kleiser's and Brinkman \& Walker's computations. Allied issues are raised by Dr F. N. Shaikh's reporting (private communications 19945 ) of contrasts in the behaviour of spanwise vortices observed in his experimental studies with Professor M. Gaster, on deepening transition in boundary layers, and by Professor C. R. Smith's experimental observations in near-wall turbulence and vortex eruptions. There seems to be a fair amount in common between these observations and the theory, for instance in Sandham \& Kleiser's (1992) noting of the short spatial and temporal scales of the spanwise vortices compared with TS scales and their use of the pressure extrema to track the appearance of vortices, in line with the current steps $1-3$. The contrasting responses of the typical vortices in step 3 (see explosions and implosions) seem in keeping with the above experimental and computational observations of vortex strengthening and weakening. 
The theory, then, is felt to provide some encouraging signs that despite its complications and subtleties much more of transition and of the internal and external dynamic-stall process can be understood and explained, and this may lead on to implications for control of the process. There is much still to be done, of course, numerically and analytically, and indeed it could turn out that shorter and shorter time and length scales come into operation subsequently (cf. \$\$4-7), including the Euler stage as in the corresponding severe break-up or in steady flow as mentioned in section 3 and as described in this section.

Thanks are due to ARO (grant nos. DAAL-03-92-G-0040 and R\&D 7728-AN-01 through Dr T. Doligalski and Dr R. Reichenbach), AFOSR (contract no. AFOSRJ-0007 through Dr A. Nachman), SERC, ESPRC, ULCC, for support of various aspects of this research, and to the referees for comments.

\section{Appendix A. On finite-time break-up in the general case}

The theory in Smith (1988a) implicitly takes the ratios $a_{1} /\left(2 a_{2}\right)$ and $-b_{1} b_{3} /\left(4 b_{4}\right)$ to be equal, i.e. that

$$
\bar{\lambda}_{2} b_{1} b_{3}+2 \bar{\lambda}_{1} b_{4}=0
$$

where the integrals $\bar{\lambda}_{1} \equiv-a_{1}, \bar{\lambda}_{2} \equiv-a_{2}$ are those used in the above paper. We show below that in fact (A 1) is true in the general case.

The main point, which has not been used previously, is simply that $t=t_{0}(T=0)$ is the first time $t$ at which the integral $I \equiv f_{0}^{\infty}(u-\hat{c})^{-2} \mathrm{~d} y$ hits zero. Hence in particular $I$ must be one-signed at the slightly previous time $t=t_{0}-\hat{\Delta}$, for all $x$, where $\hat{\Delta}$ is small and positive. Here, towards the end of step 1 ,

$$
\begin{aligned}
& u=u_{0}(y)+\hat{\Delta}^{1 / 2} u_{1}+\ldots \quad \text { [in the bulk], } \\
& \left.u=c_{0}+\hat{\Delta}^{1 / 4} b_{1} \eta+\hat{\Delta}^{1 / 2} a_{11}+\ldots+\hat{\Delta} \overline{\bar{u}}+\ldots \quad \text { [in the critical layer }\right], \\
& \hat{c}=c_{0}+\hat{\Delta}^{1 / 2} c_{1}+\ldots,
\end{aligned}
$$

and $c_{0}$ equals $c$ in $(2.4 a-d)$, while $\hat{c}$ is the inflection speed,

$$
u=\hat{c} \text { where } u_{y y}=0 .
$$

Substitution of (A 2)-(A4) into the above condition of one-signedness of $I$ then requires

$$
I=f_{0}^{\infty}\left(u_{0}-c_{0}\right)^{-2} \mathrm{~d} y+\hat{\Delta}^{1 / 2}\left\{-2 f_{0}^{\infty} \frac{\left(u_{1}-c_{1}\right)}{\left(u_{0}-c_{0}\right)^{3}} \mathrm{~d} y+\mathrm{CLB}\right\}+\ldots
$$

to be one-signed (or zero), where the first integral vanishes identically as in (1.2) and CLB is a critical-layer contribution studied below. The term in $\hat{\Delta}^{1 / 2}$ in (A 6), however, can be rewritten to give the requirement that

$$
-p_{1} \bar{\lambda}_{2}+c_{1} \bar{\lambda}_{1}+\text { CLB }
$$

is to be one-signed (or zero). The expression (A 7) follows from integrating by parts the definitions of $a_{1}, a_{2}$ just after (3.6), given (3.3b) and taking account of the finite parts. Thus $a_{1}$ becomes

$$
\begin{aligned}
a_{1} & =f_{0}^{\infty}\left(u_{0}-c\right)^{-2}\left(u_{0}^{\prime} \bar{I}-\left(u_{0}-c\right)^{-1}\right) \mathrm{d} y \\
& =2 f_{0}^{\infty}\left(u_{0}-c\right)^{-3} \mathrm{~d} y
\end{aligned}
$$


on integrating the $\left(u_{0}-c\right)^{-2} u_{0}^{\prime}$ part, where $-\bar{I}$ is the integral in (3.5), while $a_{2}$ becomes

$$
\begin{aligned}
a_{2} & =-f_{0}^{\infty}\left(\hat{\psi}_{1} \hat{u}_{1}^{\prime}-\hat{u}_{1}\left[u_{0}^{\prime} \hat{\psi}_{1}-1\right]\left[u_{0}-c\right]^{-1}\right)\left(u_{0}-c\right)^{-2} \mathrm{~d} y \\
& =-f_{0}^{\infty} \hat{\psi}_{1}\left(\frac{\hat{u}_{1}}{u_{0}-c}\right)^{\prime} \frac{\mathrm{d} y}{\left(u_{0}-c\right)}-f_{0}^{\infty} \frac{\hat{u}_{1} \mathrm{~d} y}{\left(u_{0}-c\right)^{3}} \\
& =-2 f_{0}^{\infty} \hat{u}_{1}\left(u_{0}-c\right)^{-3} \mathrm{~d} y
\end{aligned}
$$

on integrating the primed part, where $\hat{\psi}_{1} \equiv\left(u_{0}-c\right) \bar{I}=\psi_{1} / p_{1}=\tilde{\psi} / \tilde{p}$ and $\hat{u}_{1} \equiv \hat{\psi}_{1}^{\prime}=$ $u_{1} / p_{1}=\tilde{u} / \tilde{p}$ : see just after (3.6), and in $(2.4 c, d)$. Hence (A 7) is verified.

An alternative verification of (A 7) may be obtained from considering

$$
f_{0}^{\infty}\left[(u-\hat{c}) u_{\bar{x}}+v u_{y}\right](u-\hat{c})^{-2} \mathrm{~d} y
$$

along with the continuity equation $u_{\bar{x}}+v_{\bar{y}}=0$, where $\bar{x} \equiv x-\hat{c} t$. On the one hand, integration by parts shows that (A 10) is zero, since $v /(u-\hat{c})$ is zero at $y=0, \infty$ in effect. On the other hand, under the expansions (A 2), (A 4) the value of (A 10) becomes proportional to $\hat{\Delta}^{1 / 2}$ multiplied by terms in $p_{1}^{\prime}, p_{1} p_{1}^{\prime}$. These are found to reproduce the expressions (A 8), (A 9) for $a_{1}, a_{2}$, which then yield (A 7) after insertion in (A 6).

Returning to (A 7), then, we note next that $c_{1}$, CLB may be obtained explicitly. First, using (A 5) yields $\eta=\eta_{c}=\hat{\Delta}^{1 / 4} S+\ldots$ at the critical level, where $S \equiv-\left\{6 a_{13}+\bar{\tau}_{4 \eta}(\eta=0)\right\} /\left(6 b_{3}\right)$, in the notation of Smith $(1988 a)$, and $u_{y y}$ is inferred from his (2.13b) or from (A 3), (3.7a). Hence setting $u=\hat{c}$ at $\eta=\eta_{c}$ and substituting from his $(2.18 a)$ we obtain

$$
c_{1}=-\frac{2 b_{4} p_{1}}{b_{3} b_{1}}+\frac{\mu b_{1}^{4}}{6 p_{1}^{\prime}}\left\{\left(p_{1}-3 \xi p_{1}^{\prime}\right)+\frac{4 b_{4}}{b_{3} b_{1}} p_{1} p_{1}^{\prime}\right\} .
$$

Second, for CLB it is convenient to integrate $I$ by parts, giving

$$
I=-\left[\hat{c} u^{\prime}(y=0)\right]^{-1}-\int_{0}^{\infty} u^{\prime \prime}(u-\hat{c})^{-1}\left(u^{\prime}\right)^{-2} \mathrm{~d} y,
$$

with a prime denoting $\partial / \partial y$ here. Then substitution of $c_{0}$, of $c_{1}$ from (A 11) and of $u, u^{\prime}, u^{\prime \prime}$ from (A 3$)$ produces, from the $O\left(\hat{\Delta}^{1 / 2}\right)$ contributions,

$$
\mathrm{CLB}=-\frac{b_{1}^{-2}}{a_{10}^{\prime}} f_{-\infty}^{\infty} \bar{\tau}_{4 \xi} \mathrm{d} \eta=-\frac{b_{1}^{-2}}{a_{10}^{\prime}} J .
$$

Proposing now that

$$
\left(p_{1}-3 \xi p_{1}^{\prime}\right)+4 b_{4} p_{1} p_{1}^{\prime} /\left(b_{3} b_{1}\right)=0,
$$

however, we have $\bar{\tau}_{4}, c_{1 L}^{\prime}, J$ all being identically zero then, and so from (A 13)

$$
\mathrm{CLB}=o(1) .
$$

In addition, (A 11) reduces to

$$
c_{1}=-2 b_{4} p_{1} /\left(b_{3} b_{1}\right) .
$$

Therefore the form (A 7) becomes

$$
-p_{1}\left[\bar{\lambda}_{2}+2 b_{4} \bar{\lambda}_{1} /\left(b_{3} b_{1}\right)\right] \text {. }
$$


But this has to be one-signed or zero, whereas $p_{1}$ takes both positive and negative values as $\xi$ varies. Hence the coefficient in (A 17) must be zero. So, from the original general condition of one-signedness of $I$ at all times $t$ prior to $t_{0}$, the result (A 1) is derived for the general case.

We observe that the equation (2.22) of Smith (1988a) has not been assumed in the above derivation; it is a corollary, and (since $J$ is zero) it agrees with (A 14). Again, there are derivations of (A 15) alternative to that in (A 12)-(A 14). Finally here, similar working on step 2 and so on also confirms the result (A 1) for the subsequent steps.

\section{Appendix B. Further features in the terminal solution of $\S 4$}

The numerical results for the terminal system $(4.8 a-c),(4.9 a-c)$ described in $\S 4$ agree with the analytical result for small $\tilde{\mu}$ based on $g_{c 1}$ as given between $(4.8 b)$ and $(4.8 c)$. The agreement is indicated in figure $3(b)$, where for comparison purposes the analytical result takes $-\chi_{c}^{3}-\chi_{c}+6 \tilde{\mu} g_{c 1}$ with imposed boundary conditions (at $\chi_{1}, \chi_{2}$ ) the same as for the numerical ones.

The small- $\tilde{\mu}$ features prove to be the most helpful ones in the analysis in $\S 7$. It is of interest nevertheless to note also the appearance of a sub-structure developing in the terminal solutions at the other extreme, as $|\tilde{\mu}|$ approaches $\sqrt{3} / \pi(\equiv \bar{v})$. This can be seen first in the coefficients $D_{2}^{ \pm}$in $(4.9 a-c)$, which behave as

$$
D_{2}^{-} \sim 4 \bar{v}(\bar{v}-\tilde{\mu})^{-1}, \quad D_{2}^{+} \sim \frac{1}{2} \bar{v}^{-2}(\bar{v}-\tilde{\mu})^{2}
$$

as $\tilde{\mu} \rightarrow \bar{v}-$, leaving the ratio $D \sim[(\bar{v}-\tilde{\mu}) /(2 \bar{v})]^{3} \rightarrow 0$ (see figure $3 a$ ) and implying that (as mirrored in figure $3 c$ ) the left-side asymptotic growth of $g_{c}$ becomes more severe and the right-hand side milder. Consequently the entire solution acquires a left-right split, in a sense, the terms $-6, g_{c}^{\prime \prime \prime}$ in $(4.8 a)$ becoming negligible at leading order on the left and right respectively. On both the left and the right however the integral contribution in $(4.8 a)$ is dominated by the right-side range, because of the milder variation in $g$ there: see below. So on the right, where $g_{c}, \chi_{c}$ are of the orders $\hat{\delta}^{3} \hat{\varepsilon}^{-1}, \hat{\delta} \hat{\varepsilon}^{-1}$ say $(\hat{\varepsilon} \equiv \bar{v}-\tilde{\mu})$, respectively, with parameter $\hat{\delta}$ unknown, we obtain an integral equation which becomes linear on inversion as in (B 2) below and is solvable, given the required matching conditions. The right-side solution then helps to control, through the integral term, the more severe left-side response in which $g_{c}, \chi_{c}$ have the orders $\hat{\delta}^{3} \hat{\varepsilon}^{-1}, \hat{\delta}$ in turn. In between there is a near-plateau in the $g_{c}$ variation wherein the left- and right-side solutions are matched, resembling the behaviour at small $\hat{\varepsilon}$ or $D$ in the above figure, as discussed next.

At any $\tilde{\mu}$ the inversion of $(4.8 a)$ gives the equation

$$
-6 Q=\frac{1}{2} \frac{\mathrm{d}^{2}}{\mathrm{~d} Z^{2}}\left(Q^{-2}\right)+6 \tilde{\mu} f_{-\infty}^{\infty} \frac{Q(\xi) \mathrm{d} \xi}{(Z-\xi)}
$$

for $Q \equiv 1 / g^{\prime}\left(\chi_{c}\right)$ regarded as a function of $Z \equiv g_{c}$, subject to

$$
Q \sim-\frac{1}{3}\left(D_{2}^{\mp}\right)^{-1 / 3}|Z|^{-2 / 3} \text { as } Z \rightarrow \pm \infty .
$$

Here $Q$ must be negative. Various integrals of (B 2) can be taken, cf. the nonlinear integral equations in Brown et al. (1988), but (B 2) suffices for our present purposes. The small- $\tilde{\mu}$ properties described earlier can be shown to be reproduced by (B2), (B 3). The primary features for $\tilde{\mu} \rightarrow \bar{v}-$ or $D \rightarrow 0$ on the other hand are given by 
simply setting $\tilde{\mu}$ equal to $\bar{v}$ in (B 2) but altering (B 3) to the forms

$$
\begin{gathered}
Q \sim-\sigma_{1}|Z|^{-2 / 3}(\ln |Z|)^{M} \quad \text { as } \quad Z \rightarrow-\infty, \\
Q \sim-\sigma_{2} Z^{-2 / 3}(\ln Z)^{-M / 2} \quad \text { as } \quad Z \rightarrow \infty,
\end{gathered}
$$

with $M$ positive. The coefficients here satisfy $3 \sigma_{1}=2(3 \bar{v})^{2 / 3}, 3 \sigma_{2}=\frac{1}{2}(3 \bar{v})^{-1 / 3}$. Then (B 2), (B 4), (B 5) give the leading-order (scaled) solution in the matching region mentioned in the previous paragraph, subject to origin shifts in $g_{c}, \chi_{c}$, i.e. the matching solution suitably scaled is an exact solution of $(4.8 a)$ but with adjusted boundary conditions. The logarithmic terms in (B 4), (B 5) are found to be necessary when $\tilde{\mu}=\bar{v}$ and the relative increase or decrease they represent over the $|Z|^{-2 / 3}$ behaviour corresponds exactly to the left-right differences in behaviour described in the previous paragraph. Thus as $Z \rightarrow-\infty$ ( $\chi_{c}$ increasing) (B 4) balances the $6 Q$ term in (B 2) against the $\tilde{\mu}$ term but with $\xi$ occupying $(-\infty, 0)$ only, whereas for $Z \rightarrow+\infty\left(\chi_{c}\right.$ decreasing) (B 5) instead balances the nonlinear term against the $\tilde{\mu}$ one, again with $\xi$ in $(-\infty, 0)$. Higher-order terms then imply that $M=\frac{2}{3}$. Moreover, the required boundary conditions (B3) are achieved ultimately in outer regions where $|Z|$ is exponentially large, of order $\exp \left(\hat{\varepsilon}^{-2 / 3 M}\right)$ from comparison with (B 4), (B 5). The outer left-right solutions here are simple $\chi_{c}^{3}$ forms in $g_{c}$ but with logarithmic correction effects. A numerical solution for $Q$ versus $Z$ is given in figure 3(c) for a small value of $D$ and it tends to confirm the above account.

The left-right split above also reproduces the coefficient values in (B 1) as required. Further, the boundary conditions applied in the numerical solutions of $(4.8 a, c)$, $(4.9 a-c)$ suggest that the parameter $\hat{\delta}$ here is of order unity, yielding the estimate $g_{c}(0) \propto-D^{1 / 3}$ as $D \rightarrow 0$ in general, although with the particular finite boundaries used in the computations a limit of $\chi_{1}$ seems to be reached. The earlier numerical results are in fair agreement with that and with other features of the left-right split, especially given the split sub-structure which is bound to be difficult to handle numerically with accuracy. The above sub-structure and re-scaling for $|\tilde{\mu}| \rightarrow \bar{v}-$ is likely to herald a similar re-scaling occurring in terminal solutions for $|\tilde{\mu}|$ greater than $\bar{v}$.

\section{Appendix C. Large-time response for zero $\mu$}

In this Appendix we consider the large-time solution for zero $\mu$. We first pose the similarity form

and find

$$
\tilde{p}=T^{1 / 2} f(\eta), \quad \eta=X / T^{3 / 2}
$$

$$
Q(f)=\epsilon D\left(f_{\eta \eta}\right), \quad Q(f)=\frac{1}{2} f-\frac{3 \eta}{2} f_{\eta}+f f_{\eta}, \text { with } f \sim-\operatorname{sgn}(\eta)|\eta|^{1 / 3} \text { as }|\eta| \rightarrow \infty,
$$

where, for the $\mathrm{KdV}$ equation (in $\S 6$ ),

$$
D\left(f_{\eta \eta}\right)=f_{\eta \eta \eta}, \quad \epsilon=a_{4} T^{-7 / 2},
$$

whilst for the Benjamin-Ono equation (see $\S 8$ )

$$
D\left(f_{\eta \eta}\right)=\frac{1}{\pi} \int_{-\infty}^{\infty} \frac{f_{\xi \xi} \mathrm{d} \xi}{\eta-\xi}, \quad \epsilon=a_{5} T^{-2}
$$

As $T \rightarrow-\infty$ we know that

$$
\tilde{p}=|T|^{1 / 2} f^{-}\left(\eta^{-}\right), \quad \eta^{-}=X /|T|^{3 / 2},
$$


with solution

$$
\eta^{-}=-f^{-}-\left(f^{-}\right)^{3}
$$

from $\S \S 3,4$.

If, in contrast, as $T \rightarrow+\infty$ we neglect the dispersive term in (C2) we find $Q(f)=0$ with solution $\eta=f-f^{3}$, which is multi-valued for $-\frac{2}{9} \sqrt{3} \leqslant \eta \leqslant \frac{2}{9} \sqrt{3}$. We conclude that the dispersive terms must be included to give a single-valued solution; this in effect gives rise to the waves seen in the numerical solutions described in the text.

Consider now the case of the $\mathrm{KdV}$ equation. We have the conservation forms

$$
\begin{gathered}
\left(\frac{1}{2} f-\frac{3}{2} \eta f_{\eta}\right)+\left(\frac{1}{2} f^{2}-\epsilon f_{\eta \eta}\right)_{\eta}=0, \\
\frac{1}{2}\left(f^{2}-\frac{3}{2} \eta\left(f^{2}\right)_{\eta}\right)+\left(\frac{1}{3} f^{3}-\epsilon\left(f f_{\eta \eta}-\frac{1}{2} f_{\eta}^{2}\right)\right)_{\eta}=0,
\end{gathered}
$$

and we propose for a large part of the solution as $T \rightarrow \infty$ the multiple-scale expansion

$$
f \sim f_{1}(\eta, \bar{\eta})+\tilde{\epsilon} f_{2}(\eta, \bar{\eta})+\ldots, \bar{\eta}=\tilde{\epsilon}^{-1} \eta, \tilde{\epsilon}=\epsilon^{1 / 2}
$$

which from (C 2) and (C 3) yields

$$
\frac{1}{2} f_{1}-\frac{3}{2} \eta f_{1 \eta}+\tilde{\epsilon}^{-1} \frac{\partial}{\partial \bar{\eta}}\left(\frac{1}{2} f_{1}^{2}-f_{1 \bar{\eta} \bar{\eta}}-\frac{3 \eta}{2} f_{1}\right)+\frac{\partial}{\partial \eta}\left(\frac{1}{2} f_{1}^{2}-f_{1 \bar{\eta} \bar{\eta}}\right)=\frac{\partial}{\partial \bar{\eta}}(\hat{Q}) .
$$

Here $\hat{Q}$ is a combination of $f_{1}, f_{2}$ and their derivatives. The $O\left(\tilde{\epsilon}^{-1}\right)$ terms, first, give the equation governing cnoidal waves with locally constant amplitude and wavelength. Integrating twice we find

$$
\frac{1}{2} f_{1 \bar{\eta}}^{2}=F\left(f_{1}\right) \equiv \frac{1}{6} B f_{1}+A+\frac{1}{6} f_{1}^{3}+\frac{1}{2} U f_{1}^{2},
$$

where $A, B, U(\equiv-3 \eta / 2)$ are functions of the slow variable $\eta$. Second, at $O(1)$ (C 8) gives, after integration over a locally constant period $\hat{\pi}$ of the waves,

$$
\int_{\hat{\pi}} \frac{\partial B}{\partial \eta} \mathrm{d} \bar{\eta}=12 \int_{\hat{\pi}} f_{1} \mathrm{~d} \bar{\eta}
$$

governing the slow variation of $B$. Treating the second conservation law $(\mathrm{C} 6 b)$ similarly gives

$$
\int_{\hat{\pi}} \frac{\partial A}{\partial \eta} \mathrm{d} \bar{\eta}=\frac{5}{4} \int_{\hat{\pi}} f_{1}^{2} \mathrm{~d} \bar{\eta} .
$$

Hence (C9), $(\mathrm{C} 10 a, b)$, with $U=-3 \eta / 2$, control the slow variation of $A, B, U$, which in turn determine the slow variation of the waves' amplitude and period. These can be described in terms of the roots $\alpha, \beta, \gamma\left(\gamma \geqslant \beta \geqslant f_{1} \geqslant \alpha\right)$ where $6 F\left(f_{1}\right)=$ $\left(f_{1}-\alpha\right)\left(\beta-f_{1}\right)\left(\gamma-f_{1}\right)$. Then $\Delta=(\beta-\alpha)$ is the wave amplitude and the wave period $\hat{\pi}=2 \sqrt{12} K(m) /(\gamma-\alpha)^{1 / 2}$ where $m=(\beta-\alpha) /(\gamma-\alpha)$ and $K$ is the complete elliptic integral of the first kind. The wave is locally given by

$$
f_{1}=\alpha+(\beta-\alpha) \operatorname{sn}^{2}\left(\frac{(\gamma-\alpha)^{1 / 2}}{\sqrt{12}} \bar{\eta} \mid m\right),
$$

where $f_{1}$ has a local maximum at $\bar{\eta}=0$, with sn a Jacobian elliptic function, modulus $m$. The mean value of $f_{1}$ is $\bar{f}_{1} \equiv \gamma-(\gamma-\alpha) G(m) \equiv M$ where $G=E(m) / K(m)$ with $E$ the complete elliptic integral of the second kind. The coupled equations

$$
\alpha_{\eta}=\frac{9 \alpha^{2}-24 \alpha M-5(B+6 M U)}{2(\alpha-\beta)(\alpha-\gamma)},
$$




$$
\beta_{\eta}=\frac{-9 \beta^{2}+24 \beta M+5(B+6 M U)}{2(\alpha-\beta)(\beta-\gamma)},
$$

then hold, with $B=\alpha \beta+\beta \gamma+\gamma \alpha,-3 U=\alpha+\beta+\gamma$.

If $m=0$ then $(\mathrm{C} 12 a, b)$ reduce to $\alpha=\beta=M$ and $M_{\eta}=M /(3 \eta-2 M)$. This has a solution $\eta=M-c M^{3}$, for any $c$. Comparing with $(\mathrm{C} 5 b)$ ff. we are interested in the solutions with $c=1$. For small $m$ we may expand $G \sim 1-m / 2-m^{2} / 16$ to show that $\Delta$ is $O\left(\eta^{-11 / 12}\right)$ as $\eta \rightarrow \infty$ so that the amplitude of the waves decays algebraically at $+\infty$. The waves become approximately sinusoidal and the period decreases like $O\left(\eta^{-1 / 2}\right)$. These results can also be obtained by considering the form $f_{1} \sim f_{+}(\eta)+\Delta(\eta) \exp \left(\mathrm{i} T^{7 / 4} \int \phi(\eta) \mathrm{d} \eta\right)$, where $f_{+}(\eta)$ is given by $\eta=f_{+}-f_{+}^{3}$, and substituting directly into (C 2), (C 3).

As $m \rightarrow 1-$ the waveform becomes more like a solitary wave. There $\beta \rightarrow \gamma-$ and the solution takes on the singular form, as $\eta \rightarrow \eta_{s}+$,

$$
\begin{gathered}
\alpha \sim \alpha_{0}+\frac{15 \beta_{0}-9 \alpha_{0}}{12\left(\beta_{0}-\alpha_{0}\right)}\left(\eta-\eta_{s}\right)+\frac{4\left(10 \beta_{0}-7 \alpha_{0}\right)}{\left(\beta_{0}-\alpha_{0}\right)} \frac{\eta-\eta_{s}}{L}\left(1+O\left(L^{-1}\right)\right), \\
\beta \sim \beta_{0}-2 k^{1 / 2} \frac{\left(\eta-\eta_{s}\right)^{1 / 2}}{L^{1 / 2}}, \quad \gamma \sim \beta_{0}+2 k^{1 / 2} \frac{\left(\eta-\eta_{s}\right)^{1 / 2}}{L^{1 / 2}}
\end{gathered}
$$

with

$$
L=-\frac{1}{2} \ln \left(\eta-\eta_{s}\right), \quad k=2 \beta_{0}-5 \alpha_{0}, \quad \alpha_{0}+2 \beta_{0}=9 \eta_{s} / 2 .
$$

As this singularity is approached

$$
\hat{\pi} \sim \frac{\sqrt{12} L}{\left(\beta_{0}-\alpha_{0}\right)^{1 / 2}}+O(\ln L), \Delta \sim\left(\beta_{0}-\alpha_{0}\right)-\frac{12 k^{1 / 2}\left(\eta-\eta_{s}\right)^{1 / 2}}{L^{1 / 2}} .
$$

The increase in $\hat{\pi}$ here causes a breakdown in the multi-scaling (C 7), a point which is taken up below.

We envisage a solution of (C2) and (C 3$)$ in which, for $\eta$ below some value $\eta_{s}, f_{1}$ is given by $\eta=f_{1}-f_{1}^{3}$ to first order, whereas for $\eta>\eta_{s}$ the solution is wave-like, described by $(\mathrm{C} 12 a, b)$, with diminishing wave amplitude as $\eta \rightarrow \infty$, as in figure $5(b-d)$. The value of $\eta_{s}$ must be determined numerically so that $c=1$. We find $\eta_{s}=-0.058$ to three significant figures, implying $\beta_{0}=1.03, \alpha_{0}=-2.32$, and that $\Delta=3.35$, at $\eta=\eta_{s}$.

The singularity at $\eta=\eta_{s}$ is smoothed out as follows. On approaching the singularity from the right, the solution exhibits a series of solitary waves but with an increasing distance between them. The solitary waves have the form

$$
f_{1} \sim \beta_{0}-\left(\beta_{0}-\alpha_{0}\right) \operatorname{sech}^{2} \frac{\left(\beta_{0}-\alpha_{0}\right)^{1 / 2}}{\sqrt{12}} \bar{\eta},
$$

centred locally on $\bar{\eta}=0$, but the distance between successive solitons increases like $\tilde{\epsilon} \sqrt{12} L /\left(\beta_{0}-\alpha_{0}\right)^{1 / 2}$ and so the above multiple-scales solution joins with the description below. Denoting the leftmost soliton, centred on $\eta=\eta_{1}$ say, by the subscript 1 , and successive ones to the right as $2,3, \ldots$, etc., we expand

$$
f \sim \hat{f}_{0 n}\left(\tilde{\eta}_{n}\right)+\tilde{\epsilon} \hat{f}_{1 n}\left(\tilde{\eta}_{n}\right)+\ldots, \text { with } \eta=\eta_{1}+\tilde{\epsilon}\left(S_{n}+\tilde{\eta}_{n}\right),
$$

in the vicinity of the $n$th soliton, centred at $\tilde{\eta}_{n}=0$ or $\eta=\eta_{1}+\tilde{\epsilon} S_{n}$. The boundary conditions here are

$$
\hat{f}_{01} \sim \beta_{0} \text { as } \tilde{\eta}_{1} \rightarrow-\infty, \text { where } \eta_{1}=\beta_{0}-\beta_{0}^{3},
$$


Then, from substitution into $(\mathrm{C} 5 a, b)$ and solving the resulting equations, the properties

$$
\begin{gathered}
\hat{f}_{0 n}=\beta_{0}-12 r^{2} \operatorname{sech}^{2}\left(r \tilde{\eta}_{n}\right), 12 r^{2}=\left(\beta_{0}-\alpha_{0}\right), \\
\hat{f}_{1 n} \sim-\frac{\exp \left(-2 r \tilde{\eta}_{n}\right)}{16 r}\left(B_{n}+\hat{E}\right)+b_{0} \tilde{\eta}_{n}+O(1) \text { as } \tilde{\eta}_{1} \rightarrow-\infty, \\
\hat{f}_{1 n} \sim-\frac{\exp \left(2 r \tilde{\eta}_{n}\right)}{16 r}\left(B_{n}-\hat{E}\right)+b_{0} \tilde{\eta}_{n}+O(1) \text { as } \tilde{\eta}_{1} \rightarrow \infty,
\end{gathered}
$$

with $\hat{E}=2 b_{0}-5$, are obtained. Equation $(\mathrm{C} 17 a)$ fixes $B_{1}=-\hat{E}$. As $\tilde{\eta}_{n} \rightarrow+\infty$, $\hat{f}_{1 n}$ grows exponentially while $\hat{f}_{0 n}$ decays exponentially towards $\beta_{0}$. Therefore when $\tilde{\eta}_{n} \sim O\left(\ell_{n}\right), \ell_{n}=(1 / 4 r) \ln \left(r^{3} / \tilde{\epsilon} \kappa_{n}\right), \kappa_{n}=\left(B_{n}-\hat{E}\right) / 768$, we have

$$
f \sim \beta_{0}+\tilde{\epsilon}^{1 / 2} \hat{f}_{n}^{*}\left(\tilde{\eta}_{n}^{*}\right), \quad \tilde{\eta}_{n} \sim \ell_{n}+\tilde{\eta}_{n}^{*},
$$

and

$$
\hat{f}_{n}^{*}=-96\left(r \kappa_{n}\right)^{1 / 2} \cosh \left(2 r \tilde{\eta}_{n}^{*}\right)
$$

so that when $\tilde{\eta}_{n}^{*} \sim \ell_{n}$ the expansion $(\mathrm{C} 16 a, b)$ must be used, with $n$ increased by 1. The distance between successive solitons is then $\tilde{\epsilon}\left(S_{n+1}-S_{n}\right)=2 \ell_{n} \tilde{\epsilon}$. In addition $B_{n}=-2 \hat{E}\left(n+\frac{1}{2}\right)$ using $(\mathrm{C} 18 b, c)$, so that $\kappa_{n}=-\hat{E}(n+1) / 384,=(n+1) \kappa$ say. Therefore this distance decreases as $n$ increases. It becomes of the order of the soliton length scale when $n \sim r^{3} / \kappa \tilde{\epsilon}$. When this occurs $S_{n} \sim O\left(r^{2} / \kappa \tilde{\epsilon}\right)$ and so the solitons can no longer be considered close to $\eta=\eta_{1}$, the expansion (C16) becomes invalid, and (C 7)-(C 14) take over. The present description valid near $\eta=\eta_{1}=\eta_{s}$ thus matches with the multi-scaled solution valid for $\eta>\eta_{s}$. Again see figure $6(a)$.

The approach used to solve the Benjamin-Ono equation is similar. An expansion of the type

$$
f=\alpha_{0}(\eta)+\epsilon \alpha_{1}(\eta)+\ldots+\sum_{\substack{n=-\infty \\ n \neq 0}}^{n=\infty}\left(A_{0 n}(\eta)+\epsilon A_{1 n}(\eta)+\ldots\right) E^{n}
$$

where

$$
E_{\bar{\eta}}=\mathrm{i}\left(\epsilon^{-1} \gamma_{0}(\eta)+\gamma_{1}(\eta)+\ldots\right) E,
$$

with $\eta=\epsilon \bar{\eta}$ is substituted into the first two conservation forms of the equation. Solutions are found to be (see Benjamin 1967 for the first-order solution)

$$
\left.\begin{array}{c}
A_{0 n}=2 G \mathrm{e}^{-p|n|}, \hat{U}+G \Gamma=0, \quad \Gamma=\operatorname{coth} p, \hat{U}=\alpha_{0}-3 \eta / 2, \\
4 G+Q\left(\alpha_{0}\right)=0, \\
\frac{\partial\left(G^{2}\right)}{\partial \eta}=4 \alpha_{0}-\frac{15 \eta}{2} .
\end{array}\right\}
$$

These must be solved for $\eta>\eta_{s}$ such that the condition $G \rightarrow 0$ or equivalently $\eta=\alpha_{0}-\alpha_{0}^{3}$ is satisfied as $\eta \rightarrow+\infty$. We find $\eta_{s}=-0.142$.

\section{REFERENCES}

Adams, E. C., Conlisk, A. T. \& Smith, F. T. 1995 Adaptive grid scheme for vortex-induced boundary layers. AIAA J. 33, 864-870.

Aubert, X. \& Deville, M. 1983 Steady viscous flows by compact differences in boundary-fitted coordinates. J. Comput. Phys. 49, 490-509. 
Benney, D. J. \& Bergeron, R. F. 1969 A new class of nonlinear waves in parallel flows. Stud. Appl. Maths 48, 181-204.

Benjamin, T. B. 1967 Internal waves of permanent form in fluids of great depth. J. Fluid Mech. 29, $559-592$

Bodonyi, R. J., Smith, F. T. \& GaJJaR, J. S. B. 1983 Amplitude-dependent stability of boundary-layer flow with a strongly nonlinear critical layer. IMA J. Appl. Maths 30, 1-19.

Bowles, R. I. 1990 Applications of viscous-inviscid interactions in liquid layer flows and transonic boundary-layer transition. PhD thesis, University of London.

Bowles, R. I. 1995 Upstream influence and the form of hydraulic jumps in liquid layer flows on favourable slopes. J. Fluid Mech. 284, 63-96.

Bowles, R. I. \& SMith, F. T. 1992 The standing hydraulic jump: theory, computations and comparisons with experiments. J. Fluid Mech. 242, 145-168.

Brinkman, K. W. \& Walker, J. D. A. 1996 Breakup and instability in a high Reynolds number flow. AIAA Paper 96-2156.

Brotherton-Ratcliffe, R. V. 1986 PhD thesis, University of London.

Brotherton-Ratcliffe, R. V. \& Smith, F. T. 1987 Complete breakdown of an unsteady interactive boundary layer. Mathematika 34, 86-100.

Brown, S. N. \& Stewartson, K. 1978 The evolution of the critical layer of a Rossby wave, Part II. Geophys. Astrophys. Fluid Dyn. 10, 1-24.

Brown, S. N., Cheng, H. K. \& Lee, C. J. 1990 Inviscid-viscous interaction on triple-deck scales in a hypersonic flow with strong wall cooling. J. Fluid Mech. 220, 309-337.

Burns, J. C. 1953 Long waves in running water. Proc. Camb. Phil. Soc. 49, 695.

Cassel, K. W., Smith, F. T. \& Walker, J. D. A. 1996 The onset of instability in unsteady boundary layer separation. J. Fluid Mech. 315, 223-256.

Christie, I. 1985 Upwind compact finite difference schemes. J. Comput. Phys. 59.

DAVIS, D. A. R. 1992 On linear and nonlinear instability in boundary layers with crossflow. PhD thesis, University of London.

DAVIS, R. T. \& Werle, M. J. 1982 Progress on interacting boundary layer computations at high Reynolds number. In Numerical and Physical Aspects of Aerodynamic Flows (ed. T. Cebeci). Springer.

Dickinson, R. E. 1970 Development of a Rossby wave critical level. J. Atmos. Sci. 27, 627-633.

Doligalski, T., Smith, C. R. \& Walker, J. D. A. 1994 Vortex interactions with walls. Ann. Rev. Fluid Mech. 26, 573-616.

Drazin, P. G. 1983 Solitons. Cambridge University Press.

Drazin, P. G. \& Johnson, R. S. 1989 Solitons: an Introduction. Cambridge University Press.

Elliott, J. W., Cowley, S. J. \& Smith, F. T. 1983 Breakdown of boundary layers: (i) on moving surfaces; (ii) in semi-similar unsteady flow; (iii) in fully unsteady flow. Geophys. Astrophys. Fluid Dyn. 25, 77-138.

Elliott, J. W. \& Smith, F. T. 1985 On the abrupt turbulent reattachment downstream of leadingedge laminar separation. Proc. R. Soc. Lond. A 401, 1-27.

Elliott, J. W. \& Sмith, F. T. 1987 Dynamic stall due to unsteady marginal separation. J. Fluid Mech. 179, 489-512.

GaJjar, J. S. B. \& SMith, F. T. 1985 On the global instability of free disturbance with a time dependent nonlinear viscous critical layer. J. Fluid Mech. 157, 53-77.

Gaster, M. 1993 New Approaches and Concepts in Turbulence (ed. Th. Dracos \& A. Tsinober). Birkhäuser.

Greig, I. S. \& Morris, J. L. 1976 A hopskotch method for the KdV equation. J. Comput. Phys. 20, 4-80.

Gurevich, A. V. \& PitaevskiI, L. P. 1974 Nonstationary structure of a collisionless shock wave. Sov. Phys. JETP 38, 291-297.

Haberman, R. 1972 Critical layers in parallel flows. Stud. Appl. Maths 51, 139-161.

Hall, P. \& Smith, F. T. 1991 On strongly nonlinear vortex/wave interactions in boundary-layer transition. J. Fluid Mech. 227, 641-666.

Haynes, P. H. \& Cowley, S. J. 1986 The evolutions of an unsteady translating nonlinear Rossbywave critical layer. Geophys. Astrophys. Fluid Dyn. 35, 1-55. 
Hickernell, F. J. 1984 Time-dependent critical layers in shear flows on the beta-plane. J. Fluid Mech. 142, 431-449.

Higuera, F. 1994 The hydraulic jump in a viscous laminar flow. J. Fluid Mech. 274, 69-92.

HiRsh, R. S. 1975 Higher order accurate difference solutions of fluid mechanics problems by a compact differencing technique. J. Comput. Phys. 19, 90-102.

Hoyle, J. M. 1991 PhD Thesis, University of London.

Hoyle, J. M. \& Smith, F. T. 1994 On finite-time break-up in three-dimensional unsteady interacting boundary layers. Proc. R. Soc. Lond. A 447, 467-492.

Hoyle, J. M., Smith, F. T. \& Walker, J. D. A. 1991 On sublayer eruption and vortex formation. Comput. Phys. Commun. 65, 151-157.

JONES, D. P. $1994 \mathrm{PhD}$ thesis, University of Bristol.

Kachanov, Y. S., Ryzhov, O. R. \& SMith, F. T. 1993 Formation of solitons in transitional boundary layers: theory and experiment. J. Fluid Mech. 251, 273-297.

Klebanoff, P. S. \& Tidstrom, K. D. 1959 Evolution of amplified waves leading to transition in a boundary layer with zero pressure gradient. NACA Tech. Note D-195.

Klebanoff, P. S., Tidstrom, K. D. \& Sargent, L. M. 1962 The three-dimensional nature of boundary-layer instability. J. Fluid Mech. 12, 1-34.

LI, L. 1997 Numerical study of nonlinear evolution equations, using compact differencing. PhD thesis, University of London.

Lorber, P. F. \& CARTA, F. O. 1988 Airfoil dynamic stall at constant pitch rate and high Reynolds number. J. Aircraft 25, 548.

MiLes, J. W. 1981 The Korteweg-de Vries equation: an historical essay. J. Fluid Mech. 106, 131-147.

Moore, D. W. 1979 The spontaneous appearance of a singularity in the shape of an evolving vortex sheet. Proc. R. Soc. Lond. A 365, 105-119.

NishioKa, M., AsaI, N. \& IIDA, S. 1979 An experimental investigation of the secondary instability. In Laminar-Turbulent Transition, IUTAM Symp. Stuttgart. Springer.

OrszaG, S. A. \& IsRaELI, M. 1974 Numerical simulation of viscous incompressible flows. Ann. Rev. Fluid Mech. 6, 281-318.

Peregrine, D. H. 1966 Development of an undular bore. J. Fluid Mech. 25, 321-330.

Peridier, V. J., Smith, F. T. \& Walker, J. D. A. 1991a Vortex-induced boundary-layer separation. Part 1 . The unsteady limit problem $R e \rightarrow \infty$. J. Fluid Mech. 232, 99-131.

Peridier, V. J., Smith, F. T. \& Walker, J. D. A. $1991 b$ Vortex-induced boundary-layer separation. Part 2. Unsteady interacting boundary-layer theory. J. Fluid Mech. 232, 133-165.

Ryzhov, O. S. \& Sмiтн, F. T. 1984 Short-length instabilities, breakdown and initial-value problems in dynamic stall. Mathematika 31, 163-177.

Ryzhov, O. S. \& Terent'ev, E. D. 1997 A composite asymptotic model for the wave motion in a steady three-dimensional subsonic boundary layer. J. Fluid Mech. 337, 103-128.

Sandham, N. D. \& Kleiser, L. 1992 The late stages of transition to turbulence in channel flow. $J$. Fluid Mech. 245, 319-348.

SAvin, D. J. 1996 PhD thesis, University of London.

Savin, D. J., Smith, F. T. \& Allen, T. 1998 Transition of free disturbances in inflectional flow, over an isolated surface roughness. Proc. R. Soc. Lond. A (to appear).

Schubauer, G. B. \& Klebanoff, P. S. 1956 Contributions on the mechanisms of boundary-layer transition. NACA Rep. 1289.

Shaikh, F. N. \& GASTER, M. 1994 The nonlinear evolution of modulated waves in a boundary layer. J. Engng Maths 28, 55-71.

Smith, C. R., Walker, J. D. A., Haidari, A. H. \& Sobrun U. 1991 On the dynamics of near-wall turbulence. Phil. Trans. R. Soc. Lond. A 336, 131-175.

Sмith, F. T. 1977 Upstream interactions in channel flows. J. Fluid Mech. 79, 631-655.

Sмith, F. T. 1979 On the nonparallel flow stability of the Blasius boundary layer. Proc. R. Soc. Lond. A 366, 91-109.

Sмith, F. T. 1982 Concerning dynamic stall. Aero. Q. Nov. 1982.

Sмith, F. T. 1988 a Finite-time break-up can occur in any unsteady interacting boundary layer. Mathematika 35, 256-273.

Smith, F. T. 1988 b A reversed-flow singularity in interacting boundary layers. Proc. R. Soc. Lond. A 420, 21-52. 
Smith, F. T. 1991 Composite, Navier-Stokes and Euler unsteady-flow computations in boundary layers. Utd. Tech. Res. Center Rep. 91-2; also in J. Engng Maths, 1996, 30, 307-320.

SмIтH, F. T. 1993 Theoretical aspects of transition and turbulence in boundary layers. AIAA J. 31, 2220-2226.

Sмith, F. T. 1995 On spikes and spots: strongly nonlinear theory and experimental comparisons. Phil. Trans. R. Soc. Lond. A 352, 405-424.

Smith, F. T. 1996 On transition over surface roughnesses. AIAA Paper 91-1992.

Smith, F. T. \& Bodonyi, R. J. 1987 Properties of a time-dependent nonlinear viscous critical layer. Stud. Appl. Maths 77, 129-150.

Smith, F. T. \& Bowles, R. I. 1992 Transition theory and experimental comparisons on (i) amplification into streets and (ii) a strongly nonlinear break-up criterion. Proc. R. Soc. Lond. A 439, $163-175$.

Smith, F. T., Brown, S. N. \& Brown, P. G. 1993 Initiation of three-dimensional nonlinear transition paths from an inflectional profile. Eur. J. Mech. B/Fluids 12, 447-473.

Smith, F. T. \& Burggraf, O. R. 1985 On the development of large-sized short-scaled disturbances in boundary layers. Proc. R. Soc. Lond. A 399, 25-55.

Smith, F. T., Dodia, B. T. \& Bowles, R. G. A. 1994 On global and internal dynamics of spots: a theoretical approach. J. Engng Maths 28, 73-91.

Smith, F. T., Doorly, D. J. \& Rothmayer, A. P. 1990 On displacement-thickness, wall-layer and mid-flow scales in turbulent boundary layers, and slugs of vorticity in channel and pipe flows. Proc. R. Soc. Lond. A 428, 255-281.

Smith, F. T., Papageorgiou, D. T. \& Elliott, J. W. 1984 An alternative approach to linear and nonlinear stability calculations at finite Reynolds numbers. J. Fluid Mech. 146, 313-330.

Smith, F. T. \& Walton, A. G. 1989 Nonlinear interaction of near-planar TS waves and longitudinal vortices in boundary-layer transition. Mathematika 36, 262-289.

SteWARTSON, K. 1981 Marginally stable inviscid flows with critical layers. IMA J. Appl. Maths 27, $133-175$.

Timoshin, S. N. 1988 On the unsteady interaction regime in a pulsatile boundary layer. Zh. Prikl. Matem. Tekhn. Phis. 2, 23-29.

Van Dommelen, L. L. 1981 Unsteady boundary-layer separation. PhD dissertation, Cornell University.

Walker, J. D. A. $1990 a$ Wall-layer eruptions in turbulent flows. In Proc. 2nd IUTAM Symp. Structure of Turbulence and Drag Reduction, Zurich, Switzerland (ed. A. Gyr). Springer.

WALKER, J. D. A. $1990 b$ Models based on dynamical features of the wall layer. In Proc. XI US Natl Congr. of Applied Mechanics, University of Arizona, Tucson, AZ, 21-25 May 1990; Appl. Mech. Rev. 43, S232-S239.

Walker, J. D. A., Abbott, D. E., Scharnhorst, R. K. \& Weigand, G. G. 1989 Wall-layer model for the velocity profile in turbulent flows. AIAA J. 27, 140.

Walton, A. G. \& Smith, F. T. 1992 Properties of strongly nonlinear vortex/Tollmien-Schlichtingwave interactions. J. Fluid Mech. 244, 649-676.

Whitham, G. B. 1974 Linear and Nonlinear Waves. Wiley-Interscience.

Wintergerste, T. \& Kleiser, L. 1995 Direct numerical simulation of transition in a threedimensional boundary layer. Proc. Symp. on Boundary-Layer Transition Prediction in Aeronautics, Amsterdam, Dec. 1995. Springer.

ZHuK, V. I. \& RYzhOv, O. S. 1982 Locally inviscid perturbations in a boundary layer with selfinduced pressure. Dokl. Akad. Nauk SSSR 263, 56-59 (in Russian; English transl. Sov. Phys. Dokl. 27, 177-179, 1982). 